


\section{ELECTROSPINNING AS A TOOL FOR FABRICATING FUNCTIONAL CERAMICS}

Gerard Cadafalch Gazquez 
Composition of the graduation committee:

Chairman and Secretary

Promoters

Members
Prof.dr. ir. J.W.M. Hilgenkamp

Prof. dr. ir. J.E. ten Elshof

Prof. dr. ir. R.G.H. Lammertink

Prof. dr. A.J.A. Winnubst

Prof. dr. ir. K. De Clerk

Prof. dr. L. Moroni

Dr. B.A. Boukamp

\section{MESA ${ }^{+}$UNIVERSITY OF TWENTE.

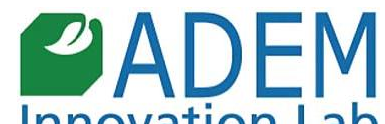

Innovation Lab

The work described in this thesis was carried out in the Inorganic Materials Science group, MESA + Institute for Nanotechnology, University of Twente, the Netherlands.

This project was financially supported by ADEM, A Green Deal in Energy Materials of the Ministry of Economic Affairs of The Netherlands (www.ademinnovationlab.nl).

Electrospinning as a tool for fabricating functional ceramics PhD thesis, University of Twente, Enschede

ISBN: 978-90-365-4254-8

DOI: $10.3990 / 1.9789036542548$

Copyright (OG. Cadafalch Gazquez

Published by: G. Cadafalch Gazquez

Cover design: Ivan Lorenzo (4 Blue Cells)

Printed by: Gildeprint, Enschede 


\title{
ELECTROSPINNING AS A TOOL FOR FABRICATING FUNCTIONAL CERAMICS
}

\section{DISSERTATION}

\author{
to obtain \\ the degree of doctor at University of Twente, \\ on the authority of the rector magnificus, \\ Prof.dr. T.T.M. Palstra,
} on account of the decision of the graduation committee, to be publicly defended on Friday, December $2^{\text {nd }}, 2016$ at 12:45

by

\section{Gerard Cadafalch Gazquez}

Born on 29 April 1986

In Vilafranca del Penedes, Catalonia, Spain. 
This dissertation has been approved by:

Prof. dr. ir. J.E. ten Elshof 


\section{Contents}

\section{Chapter 1}

Introduction

\section{Chapter 2}

Control over electrospinning of ceramics

\section{Chapter 3}

Flexible Yttrium-Stabilized Zirconia Nanofibers Offer Bioactive Cues for Osteogenic Differentiation of Human Mesenchymal Stromal Cells

\section{Chapter 4}

$\beta$-TCP Nanofiber scaffolds for bone regeneration with fine unidirectional grains

\section{Chapter 5}

Low-cost, large-area, facile and rapid fabrication of aligned ZnO nanowire device arrays

\section{Chapter 6}

Electrospun fibers as sacrificial templates to form submicron channel porosity in 3YSZ with control over pore shape, size and $\mathrm{NiO}$ catalyst deposition

\section{Chapter 7}

Conclusions and outlook

Summary

Samenvatting 
Chapter 1

Introduction 


\section{Ceramic Materials}

Ceramic materials are defined as inorganic and nonmetallic solids. They are formed by metal, nonmetal or metalloid atoms bonded together via either ionic or covalent bonds. A ceramic can be fully crystalline, semi-crystalline or completely amorphous (e.g., glasses)[1].

The properties of ceramics rely on the strong bonds between their constituent atoms, which are mostly ionic. This results in high elastic modulus and hardness, high melting points, low thermal expansion and chemical resistance. However, due to the rigidity of the chemical bonds, ceramics are naturally brittle. Furthermore, the ionic bonds naturally trap electrons, avoiding electrons to flow and providing electrical insulation for ceramic materials [2]. However, new advancements revealed the possibility of fabricating ferroelectric, semi- or superconducting ceramics $[2,3]$.

The use of ceramic materials by human being dates back to prehistorical times (Figure 1)[3]. In fact, stones (comprised of single or multiple ceramic phases) are one of the first materials ever used by human beings. Ceramic materials were initially used as figurines. Several millennia after, ceramic materials were used for other purposes too, when tiles and pottery were first made. The technology of glass making was invented later, during ancient times. During the middle ages, there was not much innovation in the ceramic industry. The only remarkable progress in this period was the invention of synthetic refractories to be used in furnaces in order to make steel, glasses, ceramics and cement [3]. The industrial 
High temperature superconductors and other electronic and biomedical 1550 applications

\section{$14,000 \mathrm{BC}$}

First tiles made in

Mesopotamia and India

\section{$8,000-5,000 \mathrm{BC}$}

Glazes discovered in Egypt
Synthetic refractories for furnaces

\section{0s}

Improved insulation and glass for automobiles

\section{0s}

Capacitors and magnetic ferrites

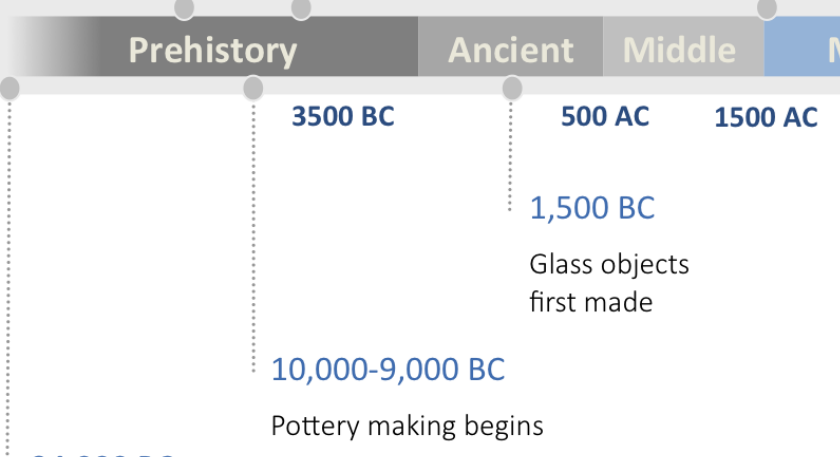

\section{4,000 BC}

Ceramic figurines used for ceremonial purposes
Mid 1800s

Porcelain electrical insulation and light bulb

1960s

High-voltage and applications for carbides and nitrides

1970s

Catalysis

Figure 1. Timeline of the history of ceramics [3].

revolution reaccelerated the progress in the field of ceramics. Engineered ceramics were started to be designed and produced in order to provide better electrical insulators and glasses. In the contemporary era, ceramic materials are being used as an essential ingredient for the development of electronics. Moreover, in the last decades ceramic materials found applications in other fields, including catalysis and biomedicine [4-8].

The classical method to fabricate ceramic parts includes sintering a powder at high temperature, aiming at the formation of a bulk piece of ceramic with a 
specific shape. Such powders consist of particles, with sizes in the range between macro and nanoscale, that can be prepared via several routes. Initially, the powders were prepared by mechanical grinding. Later, other chemical methods were also developed for finer and more homogeneous powder preparation, ranging from solid state, wet-chemical to vapor reaction-based methods. Nowadays, the powder-processing route is the most efficient and widely used method to form bulk ceramics. However, such processes do not allow enough control especially when the formation of complex microstructures is involved, as explained comprehensively by Rahaman et al. [9].

Other methods have been developed for fabricating ceramic materials in specific shapes, such as fibers or coatings in which the processing parameters and therefore, the properties of the final product can be fine-tuned. Sol-gel, chemical vapor deposition and directed oxidation techniques are commonly used examples of such processing methods [9]. The sol-gel technique is the economically most feasible method for fabricating ceramic nanofibers. The sol-gel process consists of drying and sintering a suspension of colloidal particles or a solution to form a ceramic. During the process, a 3D network containing metal ions and, commonly, organics is formed. During sintering, the organics are burnt out and the metal ions are oxidized and sintered to form continuous metal oxide ceramic bodies [9].

\section{Ceramic Nanofibers}

During the last decades, miniaturizing the size and structure of ceramic materials has become a trend in the material science community due to the superior properties that nanosized and nanostructured materials may have [4]. Among the ceramic nanostructures, nanofibers have received special interest due to their applicability in the fields of electronics, photonics, mechanics, catalysis, biomedicine and environmental sciences [4-8]. Nanofibers offer enhanced 
chemical, physical and mechanical properties due to their increased aspect ratios and large surface areas [10].

Currently there are several nanofiber fabrication methods. The most important ones are self-assembly, phase separation, force spinning, templating and electrospinning $[8,11-13]$. Most of them have limitations regarding the choice of composition, control over the process and low yield. Electrospinning is considered as a worthy alternative. Indeed, there are some commercial setups for large scale production available on the market, and several others for lab scale development [14]. Electrospinning involves a simple experimental setup that offers versatility in composition as well as a certain degree of control over fiber diameter and assembly. Moreover, electrospinning can produce nanofibers down to few $\mathrm{nm}$ in diameter while being meters long. Yet they seem to have lower production costs than other nanofiber production techniques [5, 8, 15-17].

The influence of an electric field on solutions was first studied in the 1700s [18]. The first electrospraying setups were patented in 1902 [19, 20]. Electrospinning as we know it is the outcome of some relevant studies done by Formhals in the 1930s, in which he spun cellulose and other material [21], and that resulted in the setups that we use nowadays. However, the process was forgotten until the 1990s when researchers became interested in the technique again $[16,17]$. From then on, the number of research articles and patents increased exponentially [14].

Electrospinning of ceramics is a relatively novel process. The first report is by Dai et al., who published on the fabrication of alumina-borate oxide nanofibers in 2002 [22]. Since then the method received a lot of interest among researchers. Figure 2 shows the number of papers containing the keywords "electrospinning" and "ceramic" in Scopus, a scientific web portal. It must be noted that this search also contains results that do not refer to pure ceramic nanofibers (e.g. polymeric 
fibers loaded with ceramic nanoparticles). However, a trend of shaping ceramics into nanofibers can be observed. The technique was first reported in 2003, when there were only 7 publications. In the last two years the number of publications has increased to reach over 80 in 2014 and 2015.

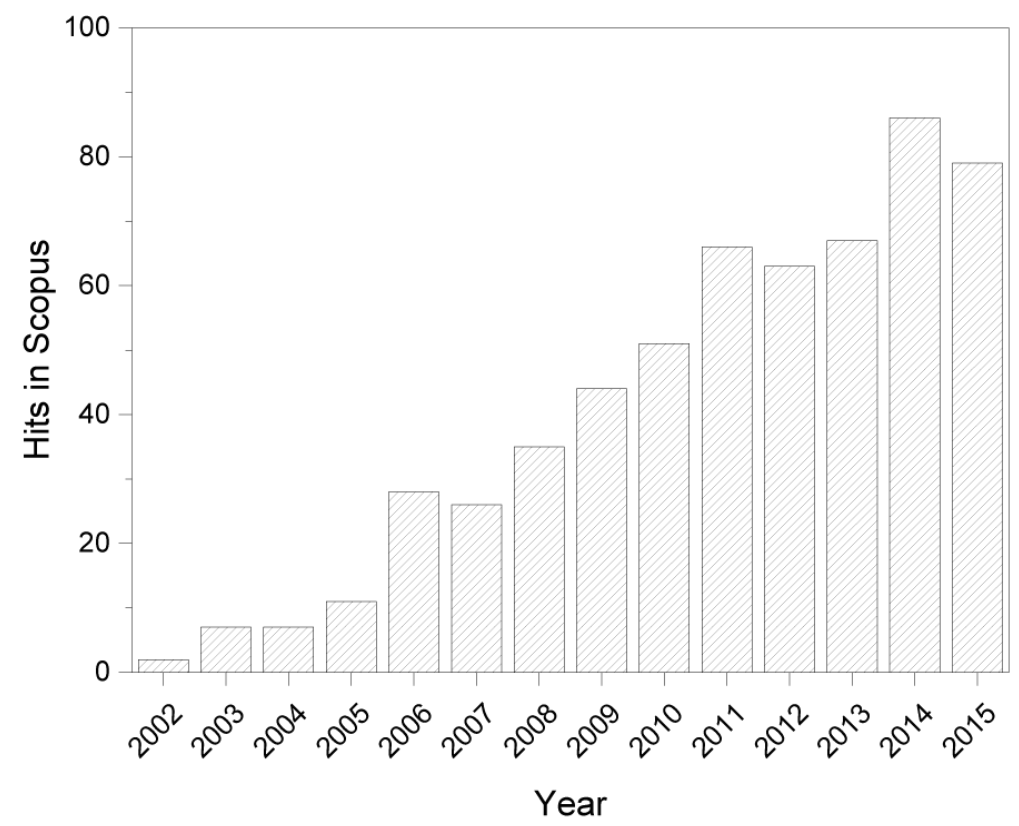

Figure 2. Hits in Scopus for "electrospinning" and "ceramic" from 2002 to 2015.

However, the current challenge in electrospinning of ceramics is to achieve better control over the resulting microstructure, and thereby, over the properties. The characteristics of electrospun micro- and nanofibers are hard to tune completely [23]. The control over fiber arrangement is not fully understood [24]. Moreover, the reproducibility must be further improved to be economically feasible to produce [4].

The goal of this research is to use electrospinning as a tool to fabricate functional ceramics while investigating the parameters controlling fiber formation, and the 
microstructural and functional characterization of the mechanical, biological and electrical properties of the resulting fibers.

\section{Electrospinning}

The electrospinning setup consists of a spinneret connected to a counterelectrode collector through a high voltage source (Figure 3. Schematic representation of electrospinning process and the different stages involved.). Normally, a polymeric solution is pumped into a nozzle, that under high electric field emits jets of micro- and nanofibers that get collected on the counter electrode $[17,23]$.

Ceramic nanofibers can be formed when a precursor is introduced in the solution. Initially, hybrid fibers are spun. Later, a heat treatment is applied to the hybrid material, the organic components are burned out and the precursors transform into oxides and sinter to form ceramic fibers [4].

Electrospinning can be described as an electrodynamic process, which is divided into four stages $[23,25]$ :
○ Jet initiation
○ Rectilinear jet
○ Bending instability
- Solidification of fiber

These stages are represented in Figure 3 where the standard electrospinning process is presented: 


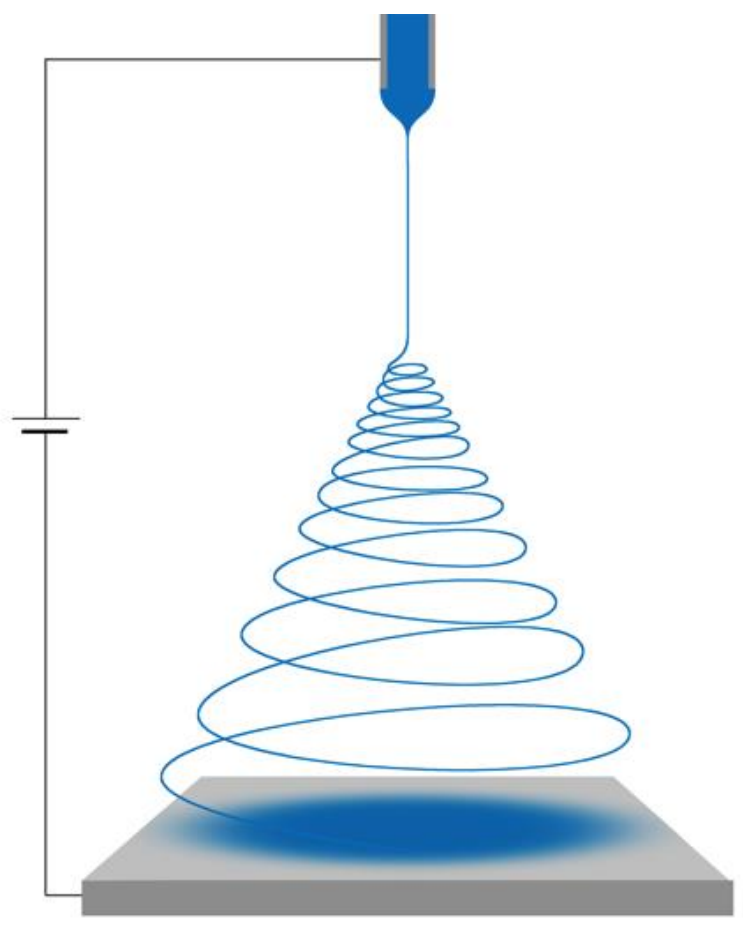

Jet Inititation

Rectiliniear Jet

Bending Instability

Fiber Solidification and Collection

Figure 3. Schematic representation of electrospinning process and the different stages involved.

\subsection{Jet initiation}

The jet is initiated in response to the electric field on the solution. That is why it is necessary to understand the processes of charge generation, transport and the forces comprised in the process to explain the jet initiation process. Studies have been done on electrospinning of polymers [23, 25-27]. Polymeric solutions are typically poorly conductive, and may also be denoted as weak electrolytes. The charges in the solution can be generated by two basic processes: unipolar emission and dissociation. Unipolar emission involves a high electric field that lowers the potential barrier to inject charges from a conductive metal to a polymer solution. Dissociation consists of the separation of ion pairs dissolved in the solution. Spontaneous dissociation occurs even without any electric field. The presence of an electric field, even at low voltages, promotes further dissociation 
of ionic species. Essentially, when an electric field is applied, there is a separation between positive and negative charges.

The repulsive Coulomb interactions between the charged elements in the fluid result in a deformed droplet. The charge with the same sign as the spinneret will be repelled from it and migrate towards the surface of the droplet that is closest to the collector electrode. When charges are introduced into the solution a balance between forces is created [28]. The charge repulsion results in an electrostatic force towards the surface of the bead. At the same time, a normal force opposite to the electrostatic force is generated due to the viscosity of the solution. The droplet will suffer further deformation upon increasing voltage, until a critical voltage is reached. A Taylor cone is then formed and a jet is emitted. At that point the electric field is so strong that charge repulsion counteracts the surface tension of the solution and a jet is formed in order to eject the excess of charge. A schematic representation in Figure 4 shows the jet emission process.
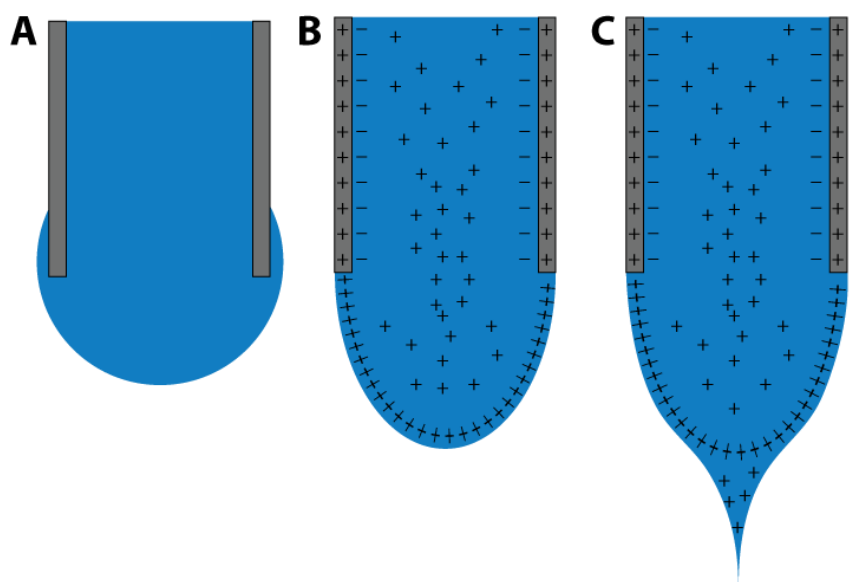

Figure 4. Charge generation and emission at the Taylor cone. a) when no electric field is applied. B) al low electric fields. C) at high electric fields. 
Therefore, the jet emission process involves a force balance between viscosity, surface tension and electric field at the Taylor cone [23, 28]. Once the process starts, a mass balance evolves between the emitted jet and the new solution pumped into the spinneret.

\subsection{Rectilinear Jet}

In the rectilinear region of electrospinning the jet moves linearly towards the collector electrode. During that step there is first a decrease in diameter with distance due to solvent evaporation and longitudinal deformation induced by the electric field. It is worth to note that electromagnetic force is dominant in the jet; gravitational forces and charge transport within the jet can be ignored [25].

\subsection{Bending instability}

The rectilinear force along the jet first increases with distance until it reaches a maximum, due to Maxwellian viscoelastic resistance. Then it starts to decrease so the bending instabilities become dominant. Finally, the jet no longer moves linearly and starts whipping forming spirals. Reneker et al. [27] reported that the viscoelastic forces along the jet and the surface tension stabilize the charges. The bending instability is triggered by perturbations of the lateral position of the jet.

During the bending instability stage, each loop of the spiral is larger in diameter and the jet undergoes further elongation. Also, there is further solvent evaporation while whipping. This results in a decrease of jet diameter [25, 27].

\subsection{Fiber solidification and collection}

During the fiber solidification step, the final loss of solvent is dominant. Solvent evaporation from an electrospinning process is thought to involve 2 mechanisms, namely conventional evaporation and ion evaporation. Conventional evaporation is a convective mass transfer as function of the partial pressure of solvent in the gas phase. Ion evaporation is the electrostatically assisted ejection of ionized 
solvent [25]. Obviously, when the jet gets thinner, the evaporation rate is higher due to the higher surface area to volume ratio.

Once the fibers are solidified, they are collected on the counter electrode where the trapped charges are released to ground [29, 30], and in the case of ceramic fibers, they are usually given a heat treatment to remove all organics and sinter the ceramic precursor into a solid object.

\section{Scope of the thesis}

The goal of this research is to understand the parameters involved in the process of electrospinning of ceramics, to learn how to control these, and finally to fabricate functional ceramic materials with tailor-made properties for various applications.

The experimental work in this thesis starts by investigating the processes governing electrospinning, as presented in Chapter 2. In this chapter, I studied the influence of solution and process parameters, as well as different setup designs on the resulting nanofibers and their mutual organization. Understanding of the relationship between the spinning process and the resulting microstructure will be fundamental for the fabrication of functional materials in a controlled manner. The knowledge gained in the experiments described in chapter 2 is appliedto fabricate functional ceramics for the fields of biomedicine, electronics and energy.

As described in Chapter $\mathbf{3}$ and Chapter $\mathbf{4}$ I fabricated ceramic nanofibers to be applied in the field of bone regeneration. In Chapter 3, yttria stabilized zirconia (YSZ) nanofiber mats behave as flexible ceramic. The microstructure and the mechanical properties of the nanofiber and nanofiber assemblies, as well as, the biochemical response of the material towards human mesenchymal stem cells were investigated,. Although YSZ is a bioinert ceramic, it provided biological cues 
for osteogenic differentiation when processed into nanofiber. Chapter 4 presents the fabrication process of $\beta$-TCP nanofiber scaffolds for the first time. I propose the usage of this material as a scaffold for bone regeneration, too, considering that $\beta$-TCP is a well-known bioactive material for bone regeneration.

Another area of application of nanofibers is electronics, as described in Chapter 5. I prepared arrays of ZnO nanofibers to form field effect transistors (FETs) and UVsensitive photodetectors. I achieved to fabricate large area nanofiber arrays in a few seconds time. Moreover, the nanofiber spacing was controlled and the nanofibers could be turned into flat ribbons.

In Chapter 6 I present a Ni-YSZ cermet with controlled porosity and nickel deposition to be used for solid oxide fuel cell anode. In this chapter, I describe the use of electrospun polymer fibers as sacrificial templates to form nanochannel porosity in a ceramic monolith. Polymeric nanofibers loaded with nickel oxide nanoparticles were spun. It is a novel method to form porous ceramics with monodisperse and ordered pores. A highly catalytic active Ni-YSZ cermet was used as model for this concept. However, the method for the preparation of porous ceramics can be applied in different fields.

Finally, in Chapter $\mathbf{7}$ the conclusions and outlook of the work are presented. The strengths and weaknesses of the electrospinning technique are assessed and the possible applications of ceramic nanofibers are evaluated as possible outlook of this thesis work. Moreover, the industrialization of nanofibers is considered. 


\section{References}

1. Ernest P. De Garmo, J.T.B., Ronald A. Kohser, DeGarmo's Materials and Processes in Manufacturing. 2011: John Wiley \& Sons.

2. American Ceramic Society. Structure and Properties of Ceramics. 2016; Available from: http://ceramics.org.

3. American Ceramic Society, History of Ceramics. 2016; Available from: http://ceramics.org.

4. Li, D., J.T. McCann, Y. Xia, and M. Marquez, Electrospinning: A Simple and Versatile Technique for Producing Ceramic Nanofibers and Nanotubes. J. Am. Ceram. Soc., 2006. 89(6): p. 1861-1869.

5. Dai, Y., W. Liu, E. Formo, Y. Sun, and Y. Xia, Ceramic nanofibers fabricated by electrospinning and their applications in catalysis, environmental science, and energy technology. Polym. Adv. Technol., 2011. 22(3): p. 326338.

6. Ramaseshan, R., S. Sundarrajan, R. Jose, and S. Ramakrishna, Nanostructured ceramics by electrospinning. J. Appl. Phys., 2007. 102(11): 111101.

7. Kim, H.W., H.E. Kim, and J.C. Knowles, Production and Potential of Bioactive Glass Nanofibers as a Next-Generation Biomaterial. Advanced Functional Materials, 2006. 16(12): p. 1529-1535.

8. Rajesh Vasita, S.S.K., Nanofibers and their applications in tissue engineering. International Journal of Nanomedicine, 2008. 1(1): p. 15-30.

9. Rahaman, M.N., Ceramic Processing, in Kirk-Othmer Encyclopedia of Chemical Technology. 2000, John Wiley \& Sons, Inc.

10. Wong, E.W., P.E. Sheehan, and C.M. Lieber, Nanobeam Mechanics: Elasticity, Strength, and Toughness of Nanorods and Nanotubes. Science, 1997. 277(5334): p. 1971-1975.

11. Papkov, D., Y. Zou, M.N. Andalib, A. Goponenko, S.Z.D. Cheng, and Y.A. Dzenis, Simultaneously Strong and Tough Ultrafine Continuous Nanofibers. ACS Nano, 2013. 7(4): p. 3324-3331.

12. Maijenburg, A.W., J. Veerbeek, R. de Putter, S.A. Veldhuis, M.G.C. Zoontjes, G. Mul, J.M. Montero-Moreno, K. Nielsch, H. Schafer, M. Steinhart, and J.E. ten Elshof, Electrochemical synthesis of coaxial TiO2-Ag nanowires and their application in photocatalytic water splitting. Journal of Materials Chemistry A, 2014. 2(8): p. 2648-2656. 
13. Altecor, A., Y. Mao, and K. Lozano, LARGE-SCALE SYNTHESIS OF TINDOPED INDIUM OXIDE NANOFIBERS USING WATER AS SOLVENT. Functional Materials Letters, 2012. 05(03): 1250020.

14. Persano, L., A. Camposeo, C. Tekmen, and D. Pisignano, Industrial Upscaling of Electrospinning and Applications of Polymer Nanofibers: A Review. Macromolecular Materials and Engineering, 2013. 298(5): p. 504520.

15. Jacobs, V., R.D. Anandjiwala, and M. Maaza, The influence of electrospinning parameters on the structural morphology and diameter of electrospun nanofibers. J. Appl. Polym. Sci., 2010. 115(5): p. 3130-3136.

16. Wu, H., W. Pan, D. Lin, and H. Li, Electrospinning of ceramic nanofibers: Fabrication, assembly and applications. Journal of Advanced Ceramics, 2012. 1(1): p. 2-23.

17. Teo, W.E. and S. Ramakrishna, A review on electrospinning design and nanofibre assemblies. Nanotechnology, 2006. 17(14): R89.

18. Gray, S., A Letter concerning the Electricity of Water, from Mr. Stephen Gray to Cromwell Mortimer, M. D. Secr. R. S. Philosophical Transactions, 1731. 37(417-426): p. 227-260.

19. Cooley, J.F., Apparatus for electrically dispersing fluids. 1902, Google Patents.

20. Morton, W.J., Method of dispersing fluids. 1902, Google Patents.

21. Anton, F., Process and apparatus for preparing artificial threads. 1934, Google Patents.

22. Dai, H., J. Gong, H. Kim, and D. Lee, A novel method for preparing ultrafine alumina-borate oxide fibres via an electrospinning technique. Nanotechnology, 2002. 13(5): p. 674-677.

23. Collins, G., J. Federici, Y. Imura, and L.H. Catalani, Charge generation, charge transport, and residual charge in the electrospinning of polymers: A review of issues and complications. J. Appl. Phys., 2012. 111(4): 044701.

24. Li, D., Y. Wang, and Y. Xia, Electrospinning of Polymeric and Ceramic Nanofibers as Uniaxially Aligned Arrays. Nano Lett., 2003. 3(8): p. 11671171.

25. Agarwal, S., A. Greiner, and J.H. Wendorff, Functional materials by electrospinning of polymers. Prog. Polym. Sci., 2013. 38(6): p. 963-991. 
26. Chang, C., K. Limkrailassiri, and L. Lin, Continuous near-field electrospinning for large area deposition of orderly nanofiber patterns. Applied Physics Letters, 2008. 93(12): 123111.

27. Reneker, D.H., A.L. Yarin, H. Fong, and S. Koombhongse, Bending instability of electrically charged liquid jets of polymer solutions in electrospinning. J. Appl. Phys., 2000. 87(9): p. 4531-4547.

28. Yarin, A.L., S. Koombhongse, and D.H. Reneker, Taylor cone and jetting from liquid droplets in electrospinning of nanofibers. J. Appl. Phys., 2001. 90(9): p. 4836-4846.

29. Lihua, L. and A.D. Yuris, Analysis of the effects of the residual charge and gap size on electrospun nanofiber alignment in a gap method. Nanotechnology, 2008. 19(35): p. 355307.

30. Chaurey, V., P.-C. Chiang, C. Polanco, Y.-H. Su, C.-F. Chou, and N.S. Swami, Interplay of Electrical Forces for Alignment of Sub-100 nm Electrospun Nanofibers on Insulator Gap Collectors. Langmuir, 2010. 26(24): p. 1902219026 


\section{Chapter 2}

\section{Control over \\ electrospinning of \\ ceramic nanofibers}

\section{The influence of solution properties, process parameter and setup design}

\section{Abstract}

The process of making ceramic nanofibers by electrospinning was studied with the aim of gaining control over the material microstructure. The study was divided in the parts: solution properties, process parameters and setup design. As a result, I identified the parameters influencing the spinnability of the solution, the fiber microstructure, and the formation of hollow or aligned fibers. Therefore, this study can be used as a guideline for the fabrication of ceramic nanofibers by electrospinning. 


\section{Introduction}

Ceramic nanofibers have gained considerable interest during the last decade. Their large surface area and aspect ratio provide superior properties for a wide range of applications such as catalysis, energy, biomedicine, sensing or electronics [1-3]. Among the different nanofiber preparation methods, electrospinning appears to be the most feasible one due to the relatively uncomplicated equipment, flexibility in composition, control over fiber characteristics and higher production rates than are possible with other methods $[1,2,4-6]$.

A typical electrospinning setup essentially consists of a spinneret connected to a counter electrode collector via a high voltage supply (Figure 1 ) [7, 8]. A viscous material solution is pumped into a nozzle. The electric field promotes the fluid to overcome the surface tension of the droplet at the tip of the spinneret, and the droplet forms a Taylor cone. The viscosity of the solution prevents the formation of separate droplets, allowing a single fiber to be drawn from the solution [7, 9]. Then, the jet shrinks in diameter to form micro and nanofibers that dry and get collected on the counter electrode $[7,8,10,11]$. The electrospinning process can be divided into 4 stages $[8,10]$ : jet initiation, rectilinear jet, bending instability, and fiber solidification and collection (Figure 1).

During the jet initiation stage, repulsive Coulomb interactions that form the Taylor's cone are dominant $[8,12]$. In the rectilinear jet stage, the viscoelastic forces and surface tension compensate for any perturbations of the jet [11]. However, at a certain point the viscosity can no longer stabilize the perturbations and whipping occurs, called the bending instability stage. At this point the jet starts whipping in a circular motion, with each consecutive circle larger than the previous one. It is considered that fiber thinning mostly happens in this stage [11]. Finally, the whipping jet reaches the collector plate and deposits the fibers on its 
surface. Upon touching the electrode, trapped electrostatic charges start being released to the ground $[8,13,14]$.

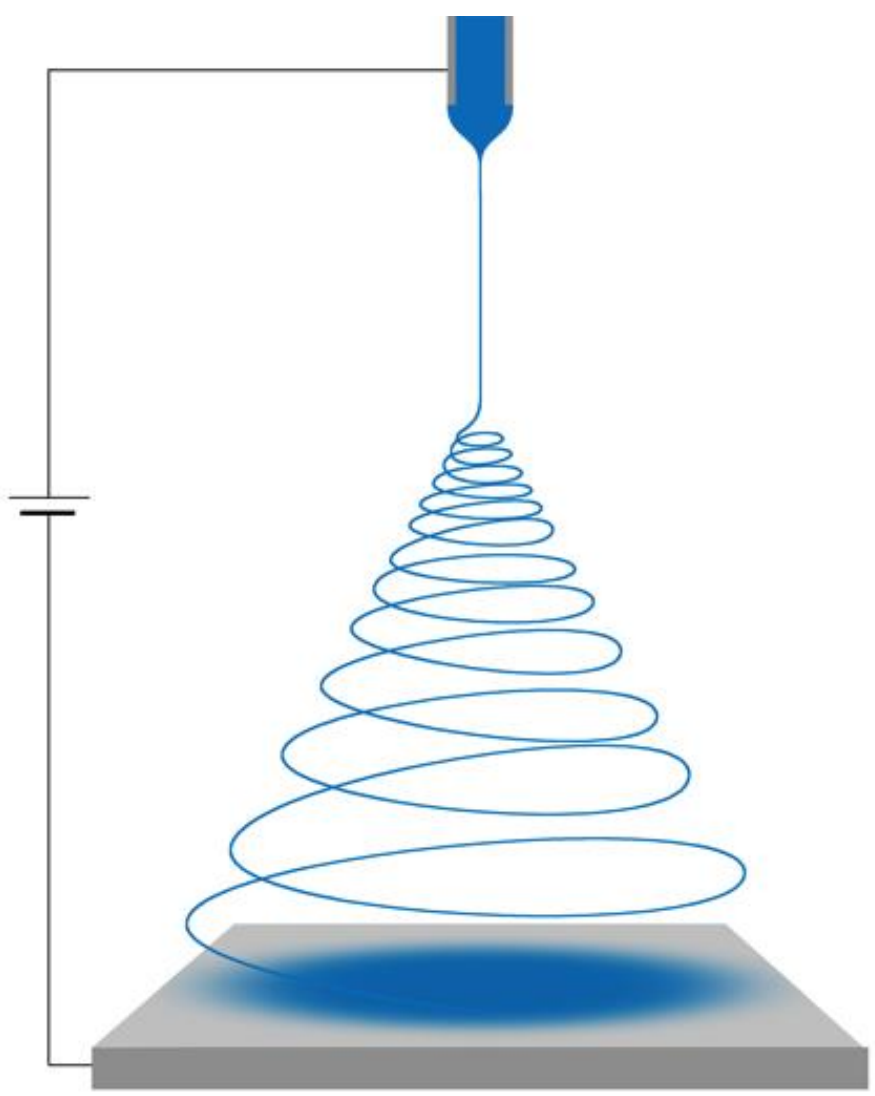

Jet Inititation

Rectiliniear Jet

Bending Instability

Fiber Solidification and Collection

Figure 1. Schematic representation of the electrospinning process.

The force balance on the jet as expressed by Equation 1 [15].

Equation 1. Force balance of the electrospinning jet [15]

$$
-p+\tau=t^{e}-\frac{\gamma}{R}
$$


where $p$ is the pressure drop related to the setup's pump, $\tau$ the viscous stress, $t^{e}$ the tension caused by the electrical field, $\gamma$ the surface tension of the solution and $R$ the jet radius. The electric force is governed by electrostatic charges in the solution, which can be correlated to the solution conductivity. The viscoelastic forces $(\tau)$ are correlated with the solution viscosity.

Ceramic nanofibers can be formed when a suitable precursor is introduced in the solution. Initially, organic-inorganic hybrid fibers are spun. In a subsequent processing step, a heat treatment is applied to the green fibers to burn out the organic compounds, and form dense crystalline ceramic fibers [1, 2]. However, achieving good control over the resulting microstructure remains a challenge and the process is not fully understood $[8,16]$. The reproducibility of the process should also be further improved in order to provide an economically feasible route to production [1].

Fabrication of ceramic nanofibers by electrospinning has been reviewed elsewhere $[1,2,17,18]$. These papers describe the fabrication of ceramic nanofibers of diverse compositions, fabrication methods and applications. Nonetheless, all theoretical and parametric studies of electrospinning and setup assemblies available so far are based on the use of polymeric fibers $[7,8]$.

The goal of this study is to investigate the degree to which control over the electrospinning of ceramics can be achieved. The study is divided in three parts: (i) solution properties, (ii) process parameters and (iii) setup design. In the first part I discuss the influence of solution properties on the final fibers. It is shown that an initially unspinnable solution can be electrospun successfully by proper control over the solution properties. A guideline to tune the solution properties for electrospinning is given. In the second part, I report the influence of process parameters on the structure and morphology of the resulting fibers. The 
experimental data are compared with literature data to further understand the process of formation of metal oxide fibers. I confirmed the applicability of theoretical studies on electrospinning of polymers on the formation of arrays of metal oxide nanofibers. Finally, in the third part, I show how to modify the setup to form hollow fibers and arrays of aligned fibers. I report the governing parameters and limitations of such techniques.

\section{Materials and Methods}

\subsection{Chemicals}

Zirconium(IV) $n$-propoxide $\left(\mathrm{Zr}\left[\left(\mathrm{OC}_{3} \mathrm{H}_{7}\right)\right]_{4}\right), 70 \mathrm{w} / \mathrm{w} \%$ in propanol) and yttrium(III)

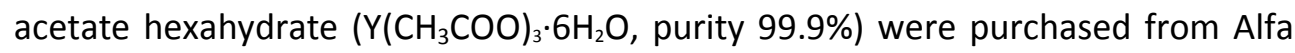
Aesar GmbH. 2-methoxyethanol (99.3\%) (2ME), and 1-propanol (99.9\%) were acquired from Sigma-Aldrich. Ethanol (99.8\%) was purchased from Atlas \& Assink Chemie B.V. Nickel(II) nitrate hexahydrate $\left(\mathrm{Ni}\left(\mathrm{NO}_{3}\right)_{2} \cdot 6 \mathrm{H}_{2} \mathrm{O}\right)$ was acquired from Merck, polyvinyl pyrrolidone (PVP, Mw 1,300,000) and 2-methoxyethanol (99.3\%) from Sigma-Aldrich and citric acid monohydrate (99.5\%) from Alfa Aesar. All chemicals were used as received.

A poly(ethylene oxide terephthalate)/poly(butylene terephthalate) (PEOT/PBT) copolymer was purchased from PolyVation BV. It consists of $45 \mathrm{wt} \%$ polyethylene oxide terephthalate and $55 \mathrm{wt} \%$ of polybutylene terephthalate. Chloroform ( $\geq$ 99\%) was acquired from Sigma-Aldrich and 1,1,1,3,3,3-hexafluoro-2-propanol (HFIP) from Biosolve.

\subsection{Electrospinning Solutions}

In this study, 3\% Yttrium Partially Stabilized zirconia (3YSZ) was taken as a model composition, prepared using an alkoxide precursor. Solution preparation was done in a nitrogen atmosphere. Briefly, zirconium $n$-propoxide and yttrium acetate were dissolved in $n$-propanol in a molar ratio of 97:6, respectively. Then, 
$5-20 \mathrm{mg} / \mathrm{ml}$ of PVP was added to the solution and the solution was left stirring overnight to complete dissolution. Finally, it was placed into a syringe connected to the electrospinning setup.

The standard solution consisted of a $0.45-0.65 \mathrm{M}$ solution of metal precursor and $10 \mathrm{mg} / \mathrm{ml}$ PVP. When the influence of polymer concentration was studied, the precursor concentration was kept at $0.65 \mathrm{M}$ and the polymer concentration was varied from 5 to $20 \mathrm{mg} / \mathrm{ml}$ PVP.

In another series of experiments I investigated the formation of nickel fibers from nickel salts. In these experiments, a $3 \mathrm{M}$ (stock) solution of $\mathrm{Ni}\left(\mathrm{NO}_{3}\right)_{2}$ in 2methoxyethanol was made and left to stir in air overnight to allow complete dissolution. Additional isopropanol was added to bring the total volume fraction of 2-ME in the final solution to 0.4 . Citric acid (CA) was added in a 6:1 molar ratio to nickel. $50 \mathrm{mg} / \mathrm{ml}$ PVP was added and the solution was diluted with $n$-propanol to bring the total concentration of $\mathrm{Ni}\left(\mathrm{NO}_{3}\right)_{2}$ to $0.21 \mathrm{M}$ (taking the volume of PVP into account). Similar $0.21 \mathrm{M}$ nickel solutions were made using ethanol, 2-ME and water. Solutions in 2-ME with CA in molar ratios CA:Ni of 2:1, 4:1 and 6:1 were also made. Finally, solutions in 2-ME containing 50, 70 or $100 \mathrm{mg} / \mathrm{ml}$ PVP were prepared.

We also performed coaxial electrospinning for the preparation of hollow ceramic fibers. I spun two immiscible solutions in two concentric needles. The outer solution was the 3YSZ precursor solution, the inner solution was a polymeric solution consisting of $200 \mathrm{mg} / \mathrm{ml}$ PEOT/PBT in a $30: 70 \mathrm{vol} / \mathrm{vol} \%$ solution of chloroform : HFIP. After spinning, the inner sacrificial polymer was removed upon thermal annealing at $850^{\circ} \mathrm{C}$ for $2 \mathrm{~h}$ so that a solid ceramic tube was formed. The annealing procedure is further explained below. 


\subsection{Fabrication Parameters}

A home-made electrospinning setup equipped with a $0.8 \mathrm{~mm}$ spinneret was used for nanofiber fabrication. The standard parameters for 3YSZ were as follows: Precursor flow rate $1 \mathrm{ml} / \mathrm{h}$; voltage $15 \mathrm{kV}$; spinneret to collector distance $20 \mathrm{~cm}$; relative humidity $30 \%$; temperature $25^{\circ} \mathrm{C}$. I varied the flow rate from 0.05 to 1 $\mathrm{ml} / \mathrm{h}$ and the voltage from 5 to $25 \mathrm{kV}$. I kept the distance from the spinneret to the collector, humidity and temperature constant to obtain a stable electrospinning process.

Besides the flat collector, I also used a grounded split electrode with an insulating gap between the electrode parts in order to obtain aligned fibers. The insulating gap distance varied from 2.0 to $7.5 \mathrm{~cm}$ and the fibers were deposited on a silicon substrate. Instead of a split electrode I also used a rotating mandrel with a radius of $3 \mathrm{~cm}$ and a speed of $1000-4000 \mathrm{rpm}$ to collect and orient fibers.

The annealing process was carried out in a convection oven at $850^{\circ} \mathrm{C}$ for $2 \mathrm{~h}$ using heating and cooling rates of $5{ }^{\circ} \mathrm{C} / \mathrm{min}$. For the thermal annealing study, samples were also annealed in a convection oven at $1{ }^{\circ} \mathrm{C} / \mathrm{min}$, or in a microwave oven at 5 ${ }^{\circ} \mathrm{C} / \mathrm{min}$ or by rapid thermal annealing. The rapid thermal annealing process involves placing the sample in a preheated microwave oven up to $850^{\circ} \mathrm{C}$.

The electrospinning parameters for the nickel precursor solution were as follows: flow rate $0.6 \mathrm{ml} / \mathrm{h}$; Voltage $15 \mathrm{kV}$; spinneret to collector distance $15 \mathrm{~cm}$; relative humidity $30 \%$; temperature $25^{\circ} \mathrm{C}$.

Coaxial spinning was performed using a spinneret from SpinBow. The inner needle had a diameter of $0.3 \mathrm{~mm}$ and the outer needle had a diameter of $0.8 \mathrm{~mm}$. 


\subsection{Characterization}

Static viscosity measurements were performed using an Anton Paar AMVn microviscometer at $25^{\circ} \mathrm{C}$, using a $3 \mathrm{~mm}$ capillary with matching $2.5 \mathrm{~mm}$ steel ball $\left(1.4034 \mathrm{~g} / \mathrm{cm}^{3}\right)$ under an $80^{\circ}$ angle. A dynamic viscosity measurement, performed on a Anton Paar Physica MCR 501, was done to prove the thinning behavior of the solutions at high shear rates.

Conductivity measurements were performed using a 2 point homemade probe. The probe consisted of 2 parallel platinum wires inserted perpendicularly to a gap in an alumina tube. The wires were fixed with a Torr Seal ${ }^{\circledR}$ epoxy resin to avoid the solution to penetrate into the tube and ensure contact with the parallel region of the wires only. The probe was connected to an Autolab PGSTAT128N potentiostat/galvanostat. The data were collected using NOVA 1.9.16 software. A frequency sweep measurement was done between $10 \mathrm{kHz}$ and $1 \mathrm{~Hz}$ with an amplitude of $10 \mathrm{mV}$. The solution was kept at $25^{\circ} \mathrm{C}$. The conductivity was calibrated with standard $\mathrm{KCl}$ solutions with known conductivities [19-21].

Scanning Electron Microscope (SEM) pictures to investigate the microstructure were taken with a ZeissMerlin Scanning Electron Microscope. Pictures of the electrospinning jet were taking utilizing a Nikon d500 camera (ISO 5000 and shutter speed of 1/200s) equipped with a Carl Zeiss $100 \mathrm{~mm}$ lens.

The surface tension of the precursor solutions was measured with the pendent droplet method using a contact angle system OCA from DataPhysics. The results were analyzed with SCA20 software. I quantified the alignment of the fibers with Fiji ImageJ software. I used optical microscope images (Nikon Eclipse ME600) images for the electrically aligned fibers and SEM pictures for the mechanically aligned fibers. The directionality tool of Fiji ImageJ provides a histogram with a 
preferred orientation and dispersion (standard deviation) over $180^{\circ}$. I defined the degree of alignment as follows:

Equation 2: Degree of Alignment

$$
\text { Degree of Alignment }=1-\frac{\text { Dispersion }}{90}
$$

We measured the charge buildup of the electrically driven alignment by monitoring the voltage between one of the ground electrodes and a platinum electrode at the center of the gap using a Keithley 197 Voltmeter.

Thermogravimetric analysis and differential scanning calorimetry were performed in a NETZSCH STA 449 F3 Jupiter Thermal analyzer. The sample was heated with a rate of $5^{\circ} \mathrm{C} / \mathrm{min}$ in air to $900^{\circ} \mathrm{C}$.

\section{Results and Discussion}

The spinning process yielded fibers as shown in Figure 2. The fibers were highly disordered and the average fiber diameter was $530 \pm 121 \mathrm{~nm}$ as determined from SEM pictures. I studied the influence of different process parameters on the resulting YSZ material, namely solution properties, process parameters and setup design. 


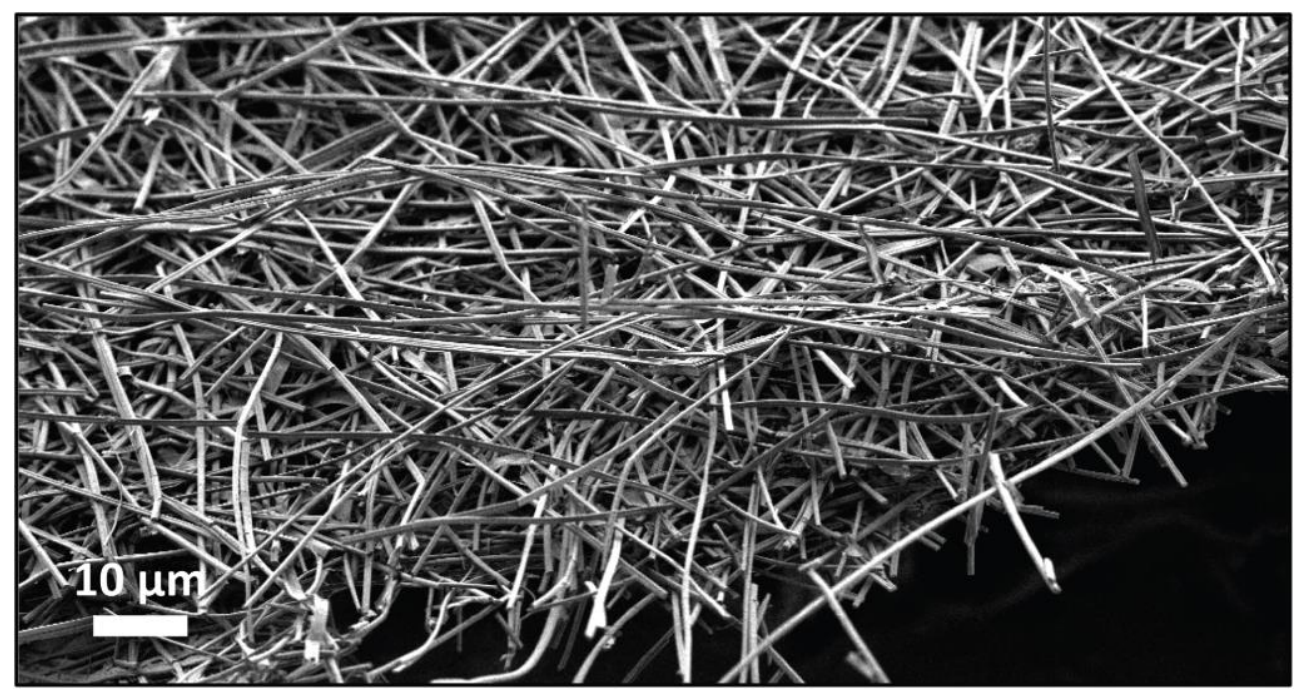

Figure 2. ) SEM picture of 3YSZ fibers. Precursor concentration $0.65 \mathrm{M}$, polymer concentration 10 $\mathrm{mg} / \mathrm{ml}$ PVP.

\subsection{Solution Properties}

The solution properties played a crucial role in the electrospinning process $[1,22$, 23]. The main solution properties affecting the electrospinning process are viscosity $\eta$, conductivity $\sigma$, surface tension $\gamma$ and solvent volatility $[8,10,11,23]$. I measured the viscosity, surface tension and conductivity of the solution experimentally. The volatility can be extrapolated from the solvent's boiling point [23-25]. I investigated the influence of both the polymer and precursor concentration on the solution properties and the resulting fibers.

The results of this experiment in which the polymer concentration was kept constant while the precursor concentration was varied between 0.45 and $0.65 \mathrm{M}$ is shown in Figure $3 \mathrm{~A}, \mathrm{~B}$. The viscosity and surface tension did not change significantly with precursor concentration. A linear correlation between the precursor concentration and fiber diameter after drying and thermal treatment is found. The fiber diameter varied from $300 \pm 44 \mathrm{~nm}$ at low precursor 
concentration, to $530 \pm 120 \mathrm{~nm}$ at high concentration. The fibers formed from the solutions with lowest precursor concentration included beads of a few micrometers in size. The conductivity increased with precursor concentration. This can be attributed to the higher concentration of free ions and other species susceptible to ionization.
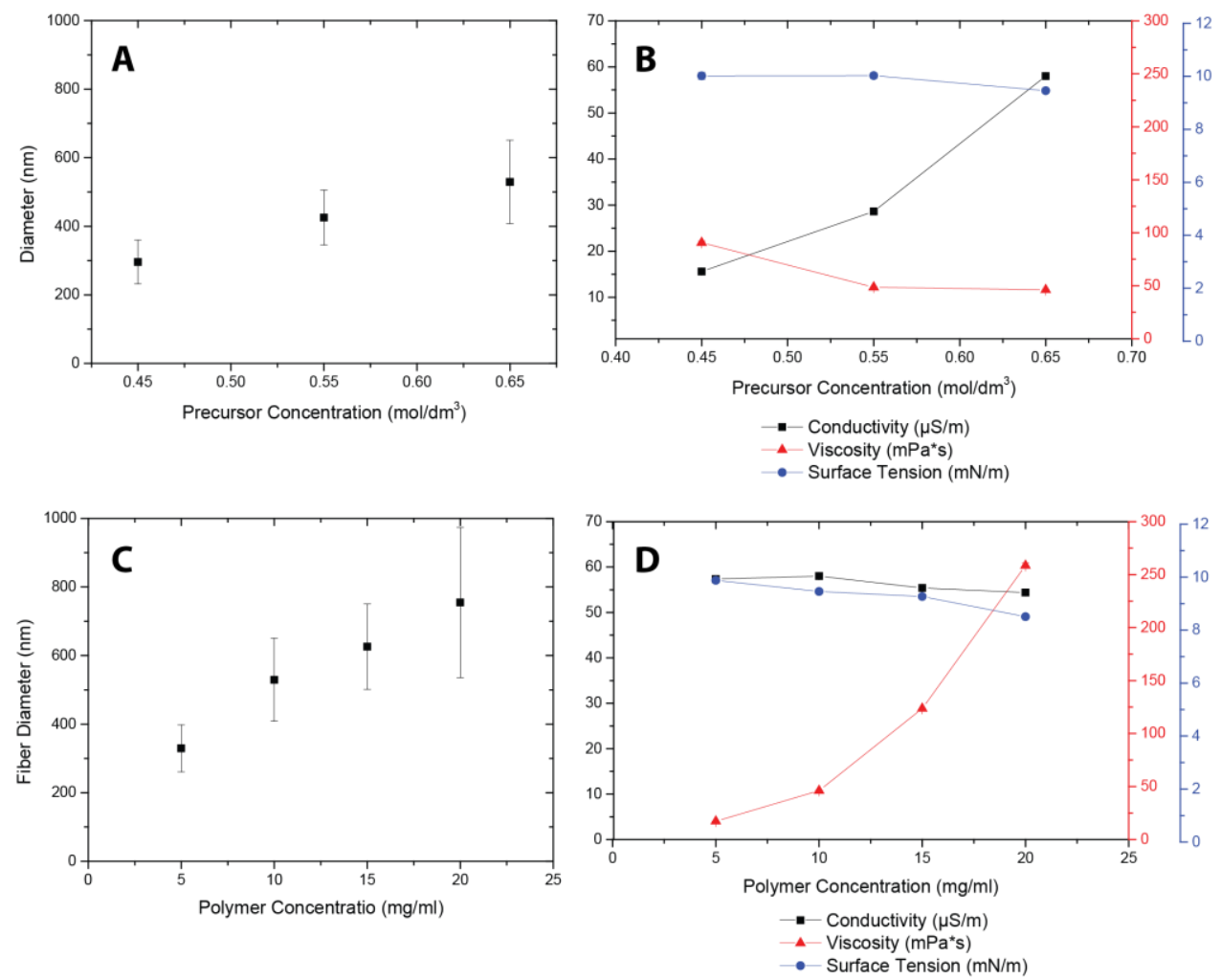

Figure 3. Influence of precursor concentration on A) fiber diameter ( $\mathrm{nm}$ ) and B) solution conductivity ( $\mu \mathrm{S} / \mathrm{m}$; Black squares), static viscosity (mPa s; red triangles) and surface tension ( $\mathrm{mN} / \mathrm{m}$; blue circles). Polymer concentration $10 \mathrm{mg} / \mathrm{ml}$. Influence of polymer concentration on $\mathrm{C}$ ) fiber diameter ( $\mathrm{nm}$ ) and D) solution conductivity ( $\mu \mathrm{S} / \mathrm{m}$; black squares), static viscosity (mPa s; red triangles) and surface tension ( $\mathrm{mN} / \mathrm{m}$; blue circles). Precursor concentration $0.65 \mathrm{M}$. 
Our results indicated that the fiber diameter is mostly dependent on the equivalent solids content after thermal annealing. Although high conductivity might result in thinner polymeric or green fibers [23], the influence of solution conductivity on fiber diameter after annealing was found to be much less prominent than the solids content. Previous studies on ceramic fibers with different composition showed the same trend $[1,16]$. Therefore, it can be expected that the solids content after annealing is the main parameter determining the diameter of ceramic nanofibers.

Beaded fibers have been attributed to low viscosities $[22,23]$ in polymer electrospinning experiments. However, in my experiments I did not observe a relationship between viscosity and the occurrence of beaded fibers. A stable jet is the result of a balance between viscoelastic, surface tension and electrostatic forces, besides the pressure drop due to the pump (Equation 1) [12, 15]. The electrostatic force is mainly dictated by the conductivity of the solution in a given electric field [8]. The beaded fibers resulted from a solution with low conductivity, which had a similar viscosity and surface tension as the solutions from which fibers without beads were made. These findings suggest that the force balance between viscosity, surface tension and conductivity was shifted so that the jet could no longer maintain a stable fiber shape, which finally resulted in the formation of beads. Indeed, previous studies have shown that formation of beaded polymeric fibers can be avoided by adding soluble salts to a solution in order to increase its conductivity $[22,26]$.

The influence of polymer concentration on solution properties and the resulting fibers was also studied. The results are presented in Figure 3 C,D. I observed an increase of fiber diameter with polymer concentration from $330 \pm 68 \mathrm{~nm}$ to $754 \pm$ $220 \mathrm{~nm}$. The viscosity of the solution increased considerably with increasing polymer concentration, while it did not have a significant influence on the 
conductivity or the surface tension. The sample with a high concentration of 20 $\mathrm{mg} / \mathrm{ml}$ polymer was too viscous given the low surface tension, and this resulted in a slightly unstable jet producing a wide range of fiber diameters. This may have been the result of a force balance shift that hampered the jet stability $[12,15]$.

We found that the influence of polymer concentration on viscosity is the main parameter that determined the fiber diameter in these experiments. A high viscosity stabilizes the jet and hinders the whipping phenomenon, which is considered to be the main cause of thinning $[10,11]$. For the sake of understanding the rheological behavior of the solutions, a dynamic viscosity measurement of the standard solution was performed. The results, presented in Figure 4, show a shear thinning behavior at high shear rates. This means that while the jet is whipping, the dynamic viscosity of the solution decreases, which allows the electrostatic instabilities to exert a larger influence. Nevertheless, it is worth mentioning that the high rate of evaporation during the whipping stage will also contribute to the rapid increase of viscosity. 


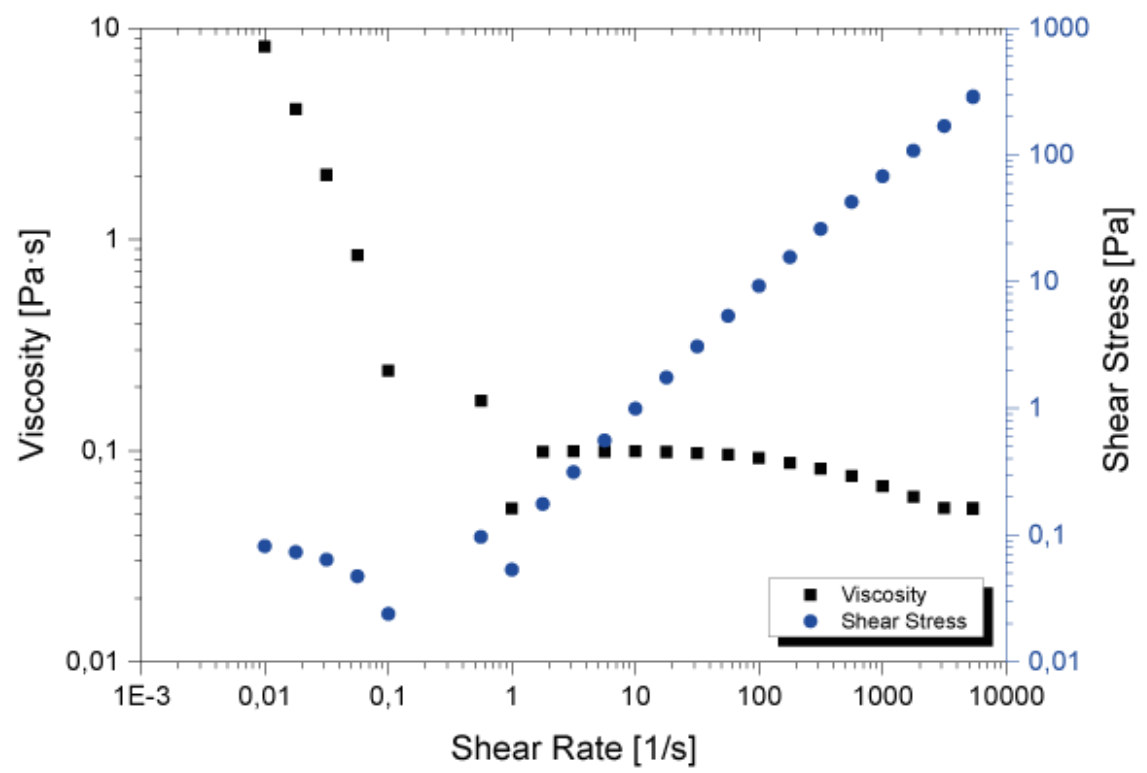

Figure 4. Dynamic viscosity data of the standard solution. Precursor concentration $0.65 \mathrm{M}, \mathrm{PVP}$ concentration $10 \mathrm{mg} / \mathrm{mL}$.

Hence, the spinnability of ceramic precursor solutions can be controlled by modifying the viscosity and conductivity of the solutions. Typically, solutions for electrospinning of ceramics contain a metal alkoxide precursor that condenses and forms a polymer-like network [1, 27]. Such solutions can be considered as weak electrolytes, similar to polymeric solutions with low conductivity [8]. In contrast, complete dissociation of salts such as metal nitrates, which are also common ceramic precursors, leads to strong electrolytes in aqueous solution. Such strong electrolytes are poorly spinnable due to their large conductivity.

The conductivity of a $0.21 \mathrm{M}$ solution of nickel nitrate in water was $60050 \pm 30$ $\mu \mathrm{S} / \mathrm{cm}$, which is 3 orders of magnitude larger than that of the $3 Y S Z$ solution (58 \pm $0.07 \mu \mathrm{S} / \mathrm{cm}$ ) discussed above. To reduce the solution's conductivity, nickel nitrate 
was dissolved in other solvents at the same concentration. In ethanol and 2-ME, the conductivity was much smaller, i.e. $5406 \pm 5 \mu \mathrm{S} / \mathrm{cm}$ and $3280 \pm 58 \mu \mathrm{S} / \mathrm{cm}$, respectively (Figure 5A). The lower conductivity is due to the lower polarity of the latter solvents compared to water, which reduces the degree of dissociation of metal salts, and the smaller acid dissociation constant of the respective solvents. Moreover, 2-ME is widely used as a complexing agent for the stabilization of metal ions and metal alkoxide precursors $[28,29]$. Complexing agents reduce the concentration of unbound ions present in the solution and, consequently, the conductivity. Citric acid was used as complexing agent to further reduce the conductivity of the nickel ion solution. Figure 5B shows a linear decrease of conductivity with increasing molar ratio of citric acid to nickel. The conductivity in 2-ME decreased from $3280 \pm 58 \mu \mathrm{S} / \mathrm{cm}$ without citric acid to $1172 \pm 3 \mu \mathrm{S} / \mathrm{cm}$ at a citric acid to nickel molar ratio of 6 .

We also investigated the influence of the PVP concentration on the nickel nitrate solution in 2-ME (without additional complexing agents). The influence of conductivity and viscosity on PVP concentration is shown in Figure 5C. Increasing the polymer concentration resulted initially in a decrease of conductivity to 1131 $\pm 4 \mu \mathrm{S} / \mathrm{cm}$ at a PVP concentration of $70 \mathrm{mg} / \mathrm{ml}$. Upon further increase of the polymer content to $100 \mathrm{mg} / \mathrm{ml}$, the conductivity increased to $2268 \pm 2 \mu \mathrm{S} / \mathrm{cm}$. This inversion of the trend can be understood by considering that the polymer (PVP) acts as a complexing agent for ions at low concentrations [30]. Hence, a decreasing conductivity is expected with increasing polymer content due to increasing degree of complexation. However, beyond a certain concentration threshold most ions are part of a complex bond with PVP, so that any further addition of polymer results in a conductivity increase, due to the fact that the polymer is ionically charged and thus contributes to the total conductivity in 
unbound form [31]. The viscosity increased with polymer concentration (Figure 5D), similar to the 3YSZ solution.

A

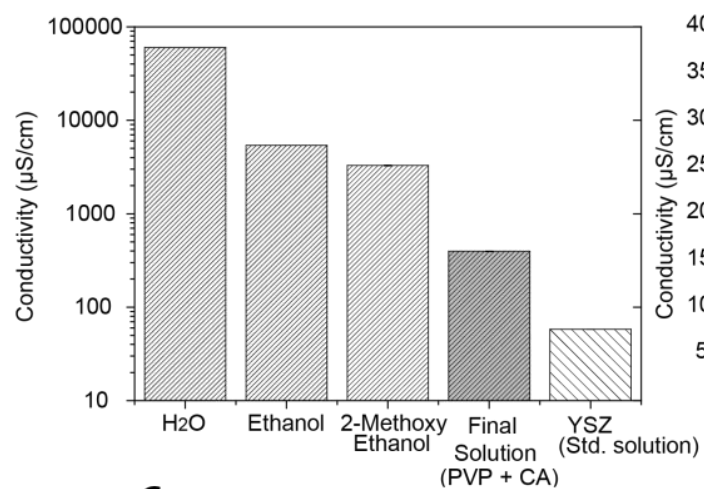

B

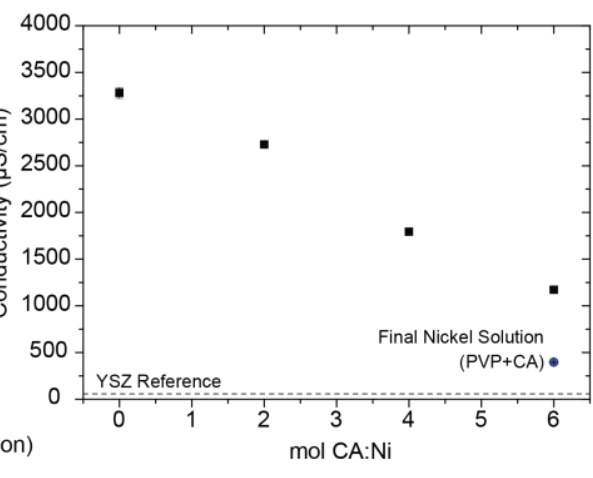

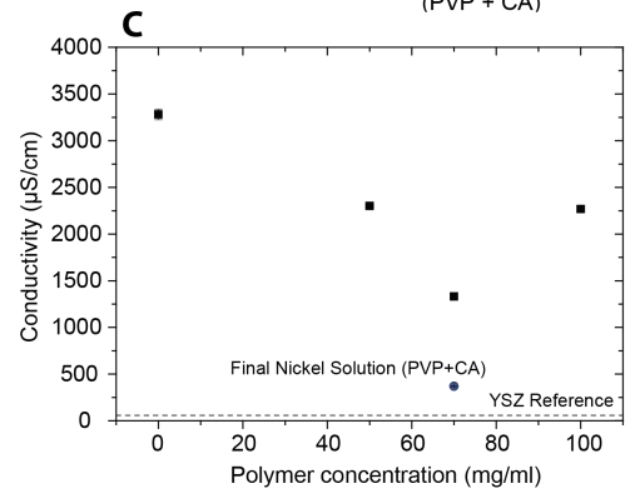

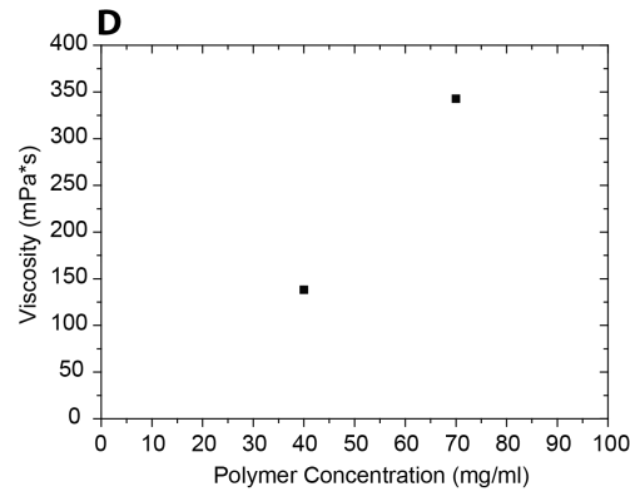

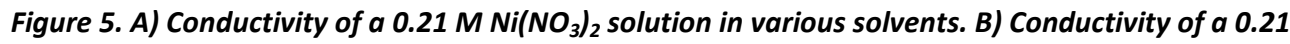
$\mathrm{M} \mathrm{Ni}\left(\mathrm{NO}_{3}\right)_{2}$ solution in 2-ME at different molar ratios citric acid (CA) : Ni. C) Conductivity of a 0.21 $\mathrm{M} \mathrm{Ni}\left(\mathrm{NO}_{3}\right)_{2}$ solution in 2-ME with different polymer concentrations. $\left.\mathrm{D}\right)$ Viscosity of a $0.21 \mathrm{M}$ $\mathrm{Ni}\left(\mathrm{NO}_{3}\right)_{2}$ solution in 2-ME with different polymer concentrations. The conductivity of the final 0.21 $\mathrm{M} \mathrm{Ni}\left(\mathrm{NO}_{3}\right)_{2}$ solution and the standard $3 Y \mathrm{YSZ}$ are also plotted as reference. E) Electrospun NiO microfibers from a $0.21 \mathrm{M} \mathrm{Ni}\left(\mathrm{NO}_{3}\right)_{2}$ solution in 2-ME with $70 \mathrm{mg} / \mathrm{ml}$ of PVP at molar CA : Ni of $6: 1$.

Nickel fibers were successfully spun by dissolving $0.21 \mathrm{M}$ of nickel nitrate into a mixture of 40:60 (by volume) of isopropanol:2ME and adding a molar ratio of nickel : citric acid of $1: 6$ and $70 \mathrm{mg} / \mathrm{ml}$ of PVP. This solution was elaborated further based on the data presented above. Moreover, the polymer concentration 
was in good accordance with a model reported by Shenoy et al., which was used to calculate the optimal polymer concentration for electrospinning [32]. I observed the formation of the Taylor cone and the deposition of material on the collector. The ceramic microfibers after collection and thermal annealing are shown in Figure 6 . However, the process was not stable for long deposition periods. The fibers tended to redissolve and form a continuous film. A solution containing the same amount of precursor and citric acid but only using $2 \mathrm{ME}$ as solvent was also processed with electrospinning. Fibers could not be obtained due to the low volatility of the solution resulting in redissolution of the spun material.

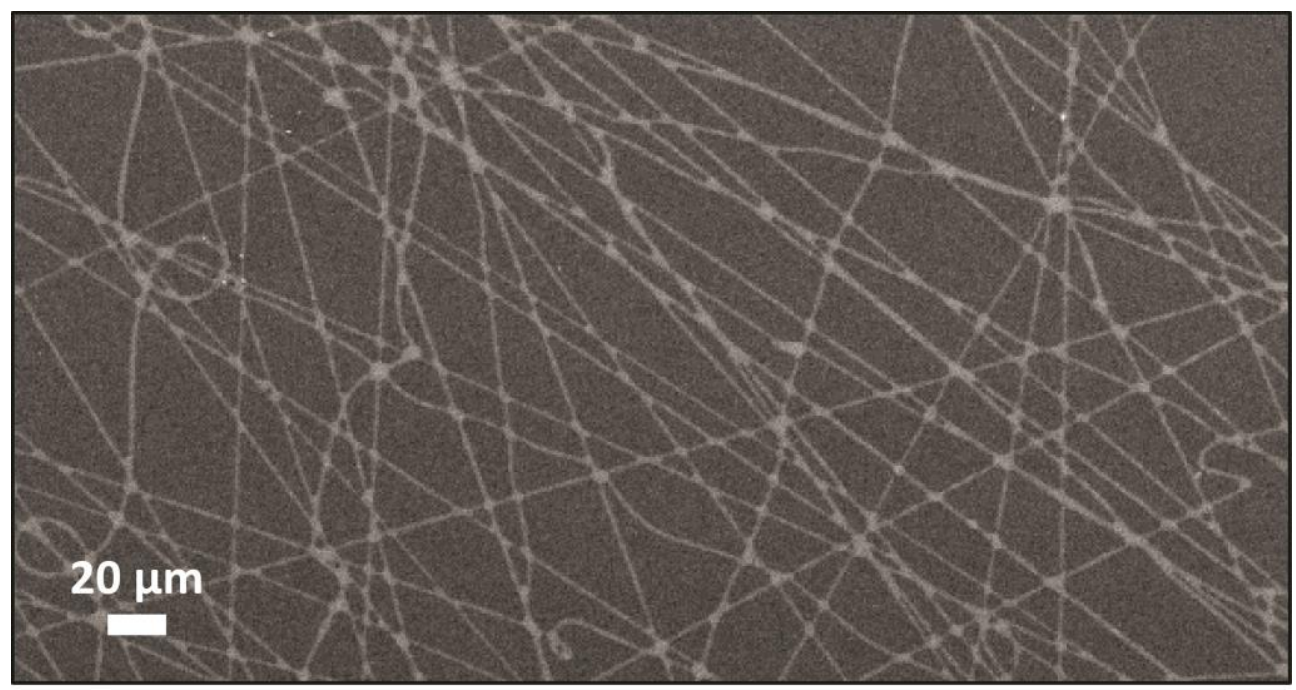

Figure 6. Electrospun $\mathrm{NiO}$ microfibers from a $0.21 \mathrm{M} \mathrm{Ni}\left(\mathrm{NO}_{3}\right)_{2}$ solution in 2-ME with $70 \mathrm{mg} / \mathrm{ml}$ of PVP at molar CA : Ni of $6: 1$.

Both conductivity and viscosity of the nickel solution were significantly higher than those of the 3YSZ solution (Table 1). However, the surface tensions of both solutions were equal. As modeled by Thompson et al. [33], the jet's momentum can be considered as a balance of electrical force counteracted by viscoelastic and surface tension forces. Both forces influence the jet's momentum proportionally. Moreover, Feng reported a force balance of the jet as expressed by Equation 1 [15], where viscosity and electrical tension are competing forces. I observed that 
the ratio between viscosity and conductivity of the nickel oxide precursor solution was very similar to the 3YSZ solution. The surface tensions of both solutions were also similar. This is consistent with the theory that a stable cone and jet are the result of a balance between viscoelastic, surface tension and electric forces [11, $12,15,33]$. The redissolution of the NiO fibers can be attributed to the low evaporation rate of the solvents and the high conductivity. The effect was more pronounced when the solvent mixture ratio $2 \mathrm{ME}$ : isopropanol was changed.

Table 1. Solution properties of 3 YSZ and NiO precursor solution. The 3YSZ solution has a precursor concentration of $0.65 \mathrm{M}$ and a PVP concentration of $10 \mathrm{mg} / \mathrm{ml}$. The NiO precursor solution has a precursor concentration of $0.21 \mathrm{M}$ in 2-ME with $70 \mathrm{mg} / \mathrm{ml}$ of PVP and citric acid in 6:1 CA : Ni molar ratio.

\begin{tabular}{lcc}
\hline & 3YSZ & NiO \\
\hline$\eta$ (mPa*s) & $46 \pm 0.15$ & $325 \pm 2$ \\
$\sigma(\mu \mathrm{S} / \mathrm{cm})$ & $58 \pm 0.07$ & $397 \pm 5$ \\
$\gamma(\mathrm{mN} / \mathrm{m})$ & $9.45 \pm 0.07$ & $9.58 \pm 0.05$ \\
\hline
\end{tabular}

To the best of my knowledge, I demonstrated here for the first time the role of viscosity and conductivity in the electrospinning process of ceramics. The observed trends are in accordance with the theoretical studies reported on the generation of jets from electrified polymeric solutions [11, 12, 15, 33]. I showed that complexation of metal ions provides a route to form suitable electrospinning solutions of high ionic strength. However, further studies should be done to improve the solution properties for metal nitrates and to obtain a fully stable electrospinning process of high quality $\mathrm{NiO}$ ceramic nanofibers 


\subsection{Process parameters}

The influence of the flow rate on the fiber diameter was investigated. A decrease of flow rate has been reported to result in smaller fiber diameters [23]. I investigated the influence of the flow rate on the rectilinear jet length and compared it with the final nanofiber diameter after collection. The increased flow rate has been thought to reduce the charge density in the jet [10, 34], thus stabilizing the rectilinear jet region [10, 11]. I observed that the rectilinear jet was longer when the flow rate was higher (Figure $7 \mathrm{~A}, \mathrm{~B}$ ). Indeed, Figure 7A shows that the fiber diameter and rectilinear jet length follow the same trend. A long stable jet at high flow rates and constant spinneret-collector plate distance implies a shorter whipping region and thus, less whipping than at lower flow rates. Since the whipping in the bending instability stage is known to be the main cause of fiber thinning [11], the jet cannot thin as much resulting in a thicker fiber.

We also investigated the effect of the electric field strength between 5 and $25 \mathrm{kV}$ on the fiber diameter and the jet length, with results as presented in Figure 7C. At $5 \mathrm{kV}$ the solution was not fully electrified. Droplets formed at the tip of the spinneret (Figure 7D) and then fell onto the mesh of green nanofibers and dissolved them. Between 10 and $20 \mathrm{kV}$ the solution was fully electrified and a stable jet was observed (Figure 7D). At $25 \mathrm{kV}$ the solution was over-electrified, which resulted in an unstable jet that eventually sprayed (Figure 7D). The fiber diameter did not change within the voltage range where a stable jet was found $(600 \pm 100 \mathrm{~nm})$. At voltages $<10 \mathrm{kV}$, the fiber diameter was $430 \pm 75 \mathrm{~nm}$. I attribute the thinner diameter to the lower speed of the jet at constant evaporation rate, which results in a shorter rectilinear jet, and thus, to a longer whipping region. Thus, it is similar to the effect of low flow rate as discussed above. At $25 \mathrm{kV}$ field strength the fiber diameter was reduced to $450 \pm 150 \mathrm{~nm}$. This is explained by considering that at very high voltages, the electric field 
stretches the jet and makes it whip further, which reduces the fiber diameter [22].

This phenomenon was confirmed by observation of a highly unstable jet (Figure 7D) with a very short rectilinear jet length.

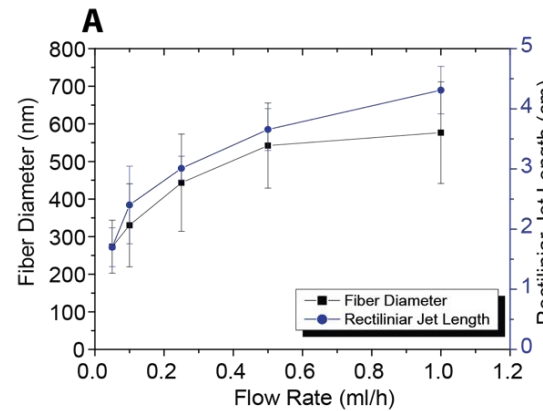

B
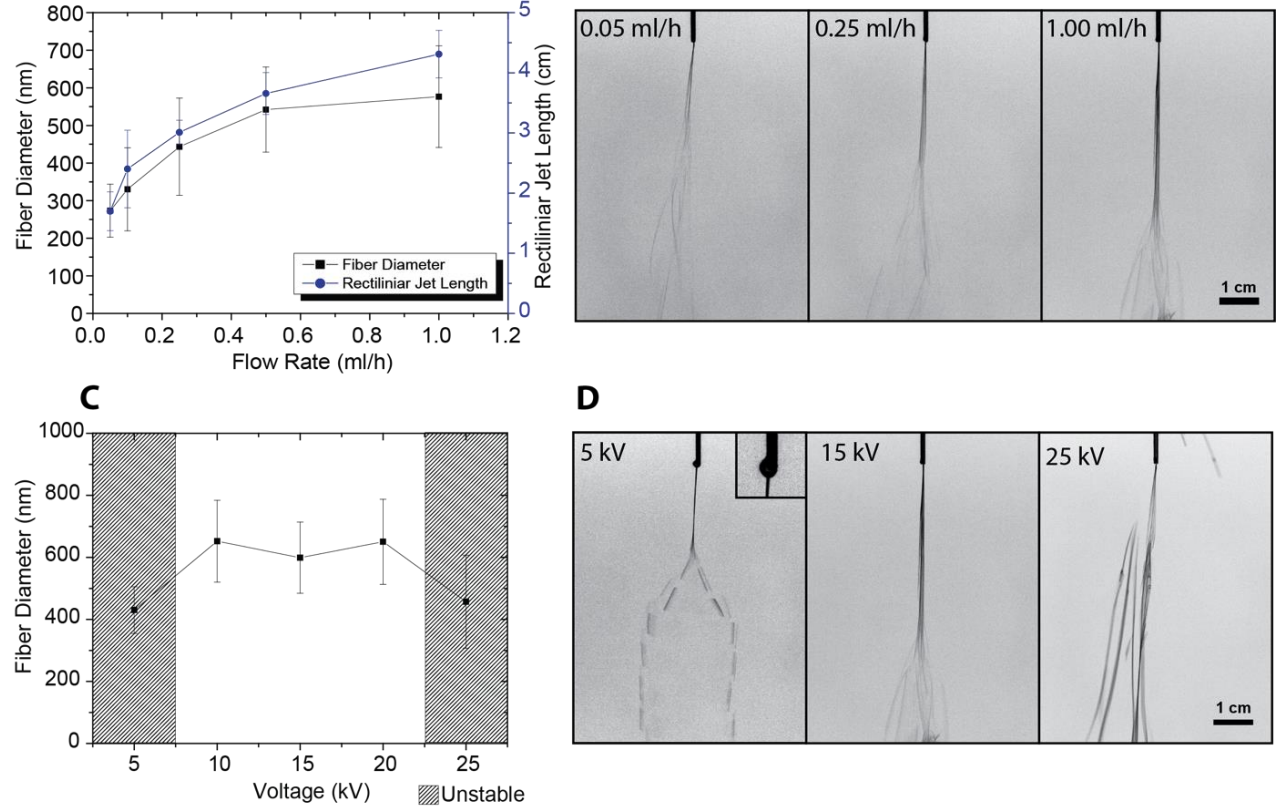

D

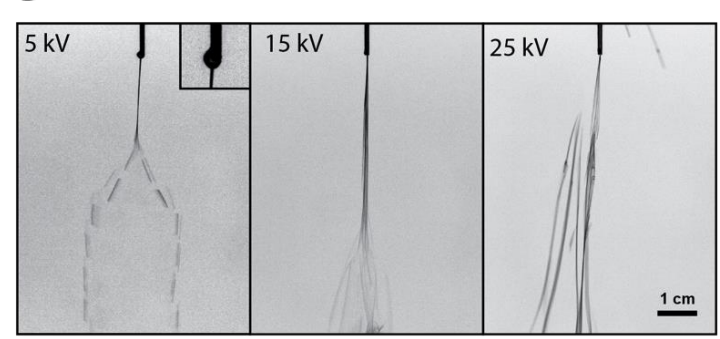

Figure 7. A) Influence of flow rate on electrospinning process. The flow rate was between 0.05 $\mathrm{ml} / \mathrm{h}$ and $1 \mathrm{ml} / \mathrm{h}$. Fiber diameter (black squares) and rectilinear jet length (blue circles) at different flow rates are shown. B) Pictures of rectilinear jet length at flow rates of $0.05,0.25$ and $1 \mathrm{ml} / \mathrm{h}$. C) Influence of potential between spinneret and collector plate on electrospinning process. Fiber diameter at flow rates ranging from 5 to $25 \mathrm{kV}$ are shown. D) Pictures of the electrospinning jet at flow rates of 5, 15 and $25 \mathrm{kV}$. Influence of annealing on the ceramic fibers.

The influence of thermal annealing was studied by changing the heating mechanism and the heating rate. The mass loss was about $45 \%$ according to thermogravimetric analysis (Figure $8 \mathrm{~A}$ ). I used either a convection oven or a microwave oven to thermally treat the fibers. The samples treated in the convection oven were heated $/$ cooled at $1^{\circ} \mathrm{C} / \mathrm{min}$ or $5^{\circ} \mathrm{C} / \mathrm{min}$. The samples treated in the microwave were heated/cooled at $5{ }^{\circ} \mathrm{C} / \mathrm{min}$ or rapid annealing was applied. 
The surface morphology varied considerably with the mode of heating and the heating rate (Figure 8B), but the fibers' diameters did not vary significantly with the nature of the thermal treatment (Figure $8 \mathrm{C}$ ).

A rougher surface was observed for the samples annealed in a convection oven than for the samples annealed in microwave oven, which can be explained in terms of the heating mechanism [35-37]. In the convection oven the heat penetrates from the ambient into the fiber, whereas in the microwave oven heat is also generated directly within the fiber. Microwave heating has been reported to produce denser and smoother ceramic thin films than when prepared in a convection oven [36-41]. The precise mechanism of sintering using microwave radiation is not well understood and is often called the "microwave effect" [36, 42]. The smoothness of the fibers annealed by microwave heating is attributed to sudden shrinkage and densification, decreased step-bunching mechanism and/or enhanced oxygen mobility due to microwave radiation $[36,40,41]$. 
A

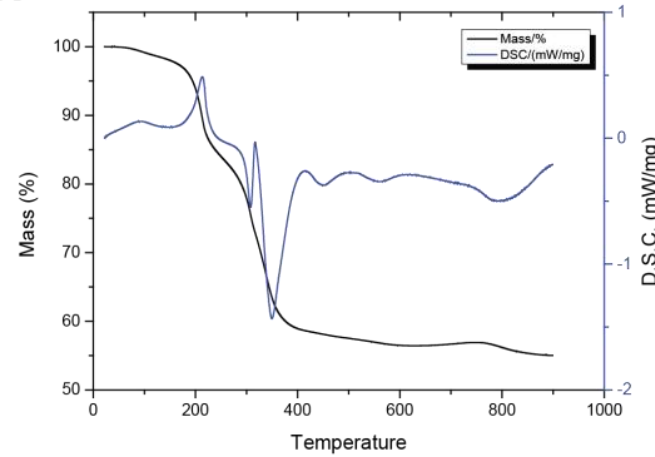

C

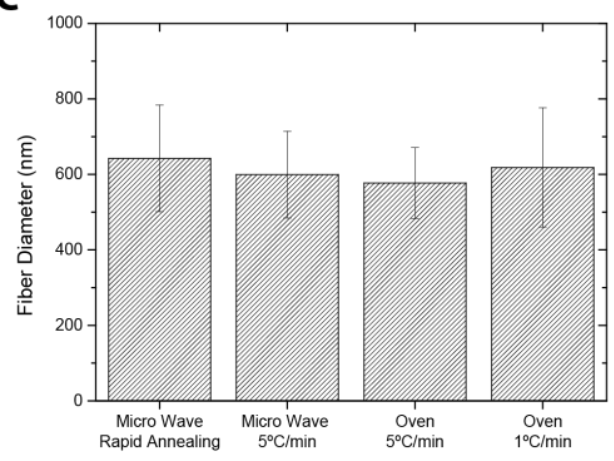

B

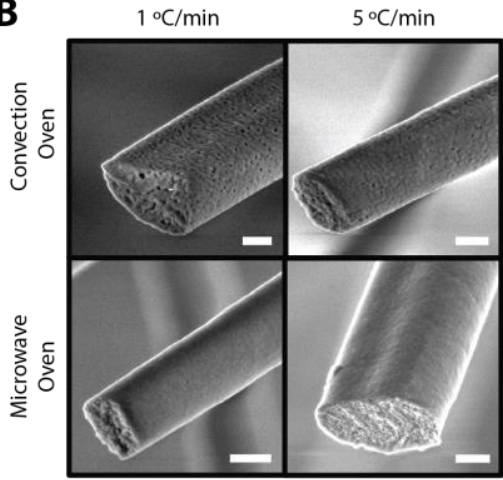

D

$5^{\circ} \mathrm{C} / \mathrm{min}$

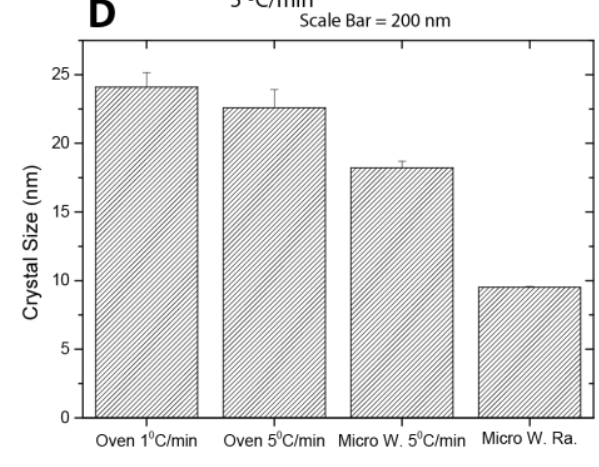

Figure 8. A) Thermogravimetic analysis and differential scanning calorimetry $\left(5^{\circ} \mathrm{C} / \mathrm{min}\right.$ in air). B) Surface morphology of ceramic fibers after different annealing procedures with different heating/cooling rates and heating mechanism (convection oven or microwave heating). C) Fiber diameter of ceramic fibers after different annealing procedures with different heating/cooling rates and heating mechanism (convection or microwave radiation). D) Crystallite size of ceramic fibers after different annealing procedures with different heating/cooling rates and heating mechanism (convection oven or microwave heating).

The heating rate affected the grain size, as shown in Figure 8D. The sample heattreated by rapid annealing had the smallest crystal size, $9.5 \pm 0.1 \mathrm{~nm}$, whereas the sample with a slow heating rate of $1^{\circ} \mathrm{C} / \mathrm{min}$ had the largest size, $24 \pm 1.0 \mathrm{~nm}$. The samples anneald at $5{ }^{\circ} \mathrm{C} / \mathrm{min}$ had intermediate crystal sizes, i.e. $18 \pm 0.5 \mathrm{~nm}$ for the microwave samples and $22 \pm 1.3 \mathrm{~nm}$ for the samples from the convection oven. The differences in grain size are attributed to the effective annealing time. The lower the heating rate, the longer the sample will be at a temperature where 
grain growth can occur. The effect can be substantial since the samples were treated at $850{ }^{\circ} \mathrm{C}$, while crystal formation already started at $400{ }^{\circ} \mathrm{C}$ according to differencial scanning calorimetry (Figure 8D). However, the difference in grain size between the two samples heated and cooled at $5^{\circ} \mathrm{C} / \mathrm{min}$ can only be attributed to differences in heating mechanism. Xie et al. reported a smaller and more uniform grain size when zirconia was sintered in a microwave oven [37]. The nanofibers presented here may have undergone a similar process.

\subsection{Setup}

The setup design was modified to form hollow fibers and arrays of aligned fibers. The section is divided it into two subsections, spinneret and collector, as the two core parts of the setup.

\subsubsection{Spinneret}

Ceramic hollow fibers were formed by changing the single needle spinneret for two concentric needles (Figure 9A). In this way two different solutions can be spun simultaneously. The 3YSZ precursor solution was pumped through the outer needle and an immiscible polymer solution (PEOT/PBT) through the inner needle. Upon annealing, the inner polymer was burnt out and hollow fibers formed. The outer flow rate was kept constant at $1 \mathrm{ml} / \mathrm{h}$ and the inner flow rate was varied from $0.2 \mathrm{ml} / \mathrm{h}$ to $1 \mathrm{ml} / \mathrm{h}$. At flow rates below $0.4 \mathrm{ml} / \mathrm{h}$ hollow fibers could not be made as the inner polymer content was not enough to maintain a hollow fiber geometry in that situation. Figure $9 \mathrm{~B}$ shows the morphology of the resulting fibers. Dense fibers with isolated porosity seem to have been formed instead of continuous hollow fibers. 
a)

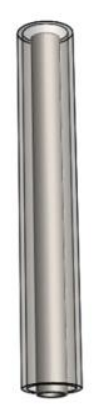

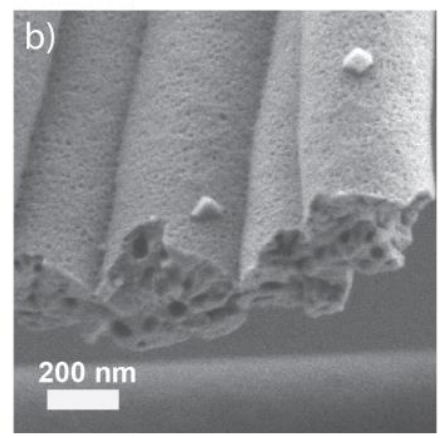
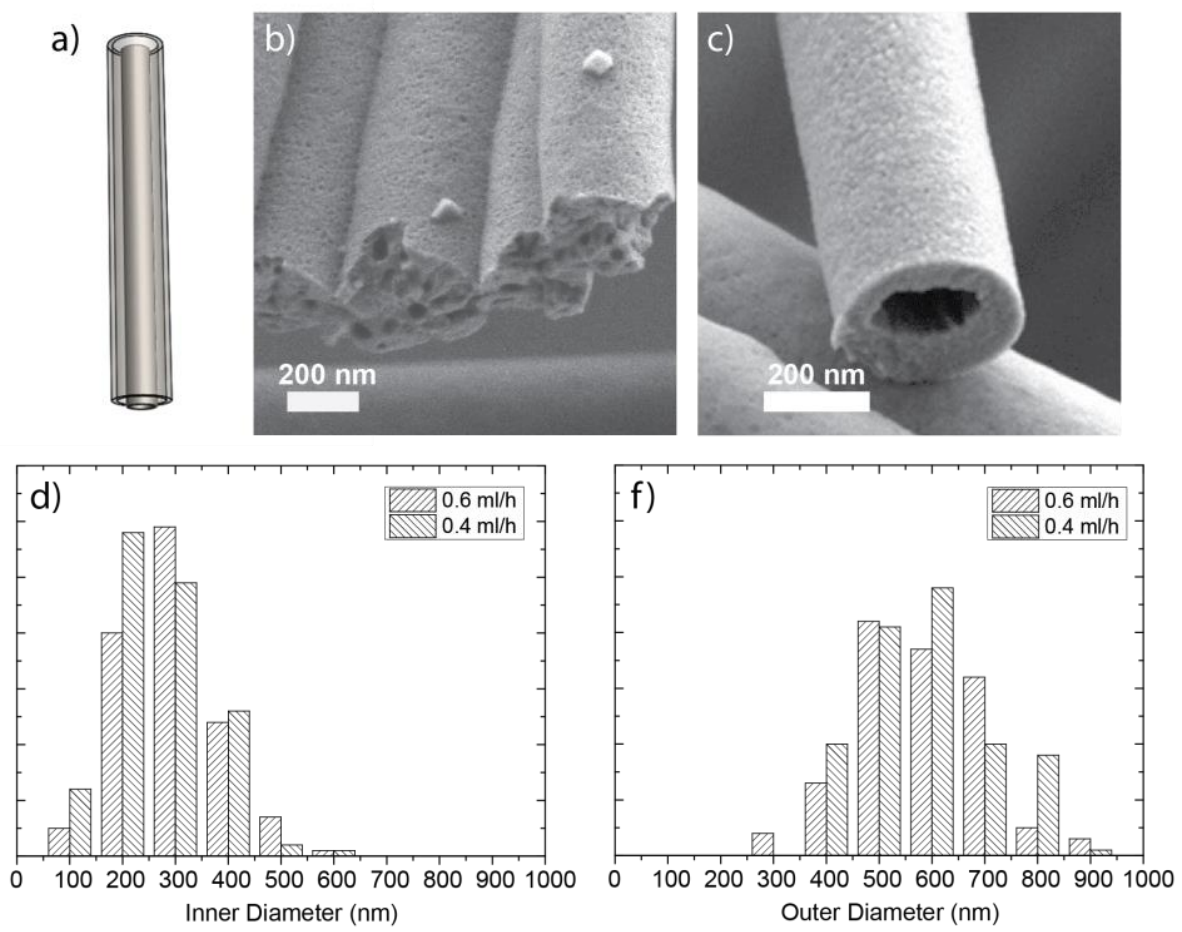

Figure 9. A) Coaxial spinneret. B) Coaxial spinning of porous fibers at an inner flow rate of $0.2 \mathrm{ml} / \mathrm{h}$ of PolyActive solution and an outer flow rate of $1 \mathrm{ml} / \mathrm{h}$ of $3 Y S Z$ solution; hollow fibers are not formed. c) Hollow fiber made by coaxial electrospinning. The inner flow rate was $0.6 \mathrm{ml} / \mathrm{h}$ of PolyActive solution and the outer flow rate rete was $1 \mathrm{ml} / \mathrm{h}$ of $3 Y S Z$ solution. C) Frequency distribution of the inner hollow fiber diameter at two inner flow rates: 0.4 and $0.6 \mathrm{ml} / \mathrm{h}$ of PolyActive solution, the outer needle carried a flow rate of $1 \mathrm{ml} / \mathrm{h}$ of $3 Y S Z$ precursor solution. D) Frequency distribution of the outer hollow fiber diameter under the same conditions. The fibers shown were thermally treated at $850^{\circ} \mathrm{C}$.

Ceramic hollow fibers were only formed at inner flow rates between 0.4 and 0.6 $\mathrm{ml} / \mathrm{h}$ (Figure 9C) $\mathrm{m}$ when the outer flow rate was kept constant at $1 \mathrm{~mL} / \mathrm{h}$. In this regime the inner flow rate did not have an influence on the final diameter within experimental error. The annealed fibers had an outer diameters of $530 \pm 128 \mathrm{~nm}$ and inner diameters of $230 \pm 93 \mathrm{~nm}$ (Figure $9 \mathrm{D}, \mathrm{F}$ ). At inner flow rates above 0.6 
$\mathrm{ml} / \mathrm{h}$, the two immiscible solutions formed an emulsion and the jet became unstable.

\subsubsection{Collector}

A

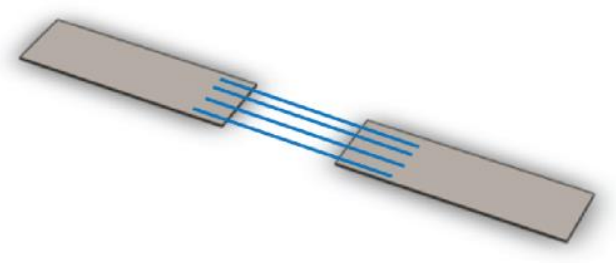

B

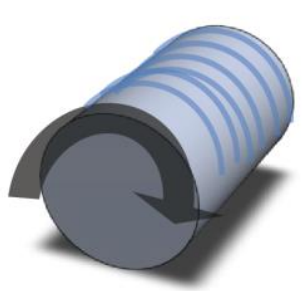

Figure 10. A-B) Collectors for ceramic nanofiber alignment; A) Electrically driven alignment: two ground electrodes with an insulating gap; B) Mechanically driven alignment: rotating mandrel as ground electrode.

Electric field-driven alignment was studied by modifying the collector in order to have two connected ground electrodes with a gap in between them, as shown in Figure 10A. The gap distance and flow rate were varied to investigate the influence on the degree of alignment. The principle of mechanically driven alignment, discussed in more detail below, is shown in Figure 10B. 
Studies on electrospinning of polymers showed the influence of the gap distance on the alignment of fibers [13, 43]. Through simulations, it has been demonstrated that the lateral force by the electric field increases with gap distance, which favors fiber alignment. The results of an experiment in which the flow rate was $0.5 \mathrm{ml} / \mathrm{h}$ and the gap distance was varied from 1.0 to $7.5 \mathrm{~cm}$ is shown in Figure 11A-B. At shorter gap distances the degree of alignment was lower than at larger gap distances. This trend is similar to previously reported data $[13,43]$. Therefore, the results from studies on polymeric electrospinning seem to be also applicable to arrays of ceramic nanofibers.
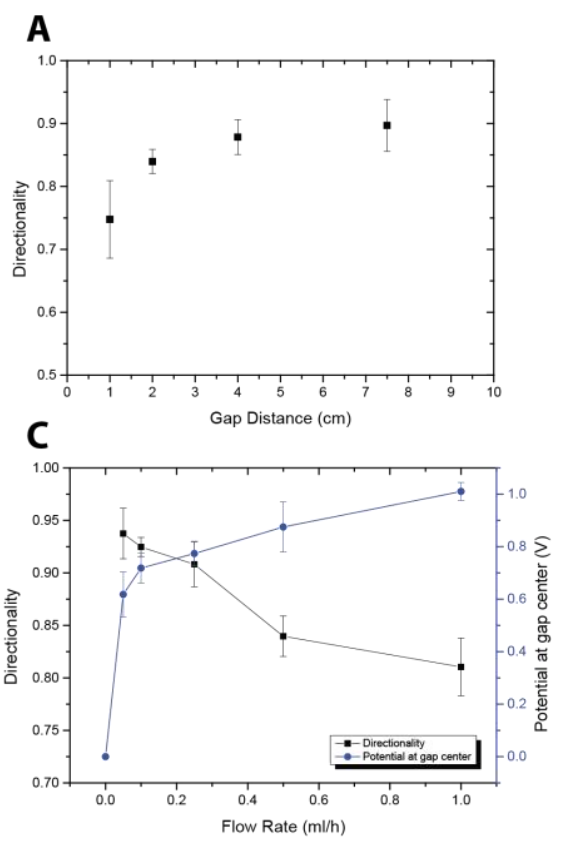

B

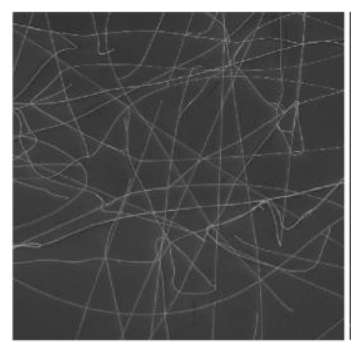

D
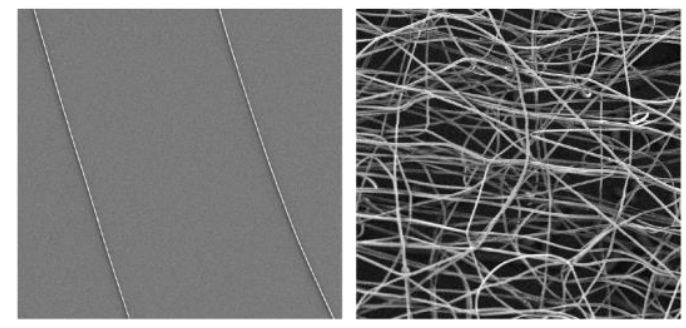

Figure 11. A-B) Influence of gap distance on degree of alignment of thermally annealed fibers after $15 \mathrm{~s}$ of deposition and at a flow rate of $0.25 \mathrm{ml} / \mathrm{h}$ of $3 Y S Z$ precursor solution; A) Degree of alignment versus gap distance ranging from $1 \mathrm{~cm}$ to $7.5 \mathrm{~cm}$; B) SEM pictures of the samples spun with gap distances of 1.0 and $7.5 \mathrm{~cm}$. C-D) Influence of flow rate of $3 Y S Z$ precursor solution on degree of alignment of fibers after $15 \mathrm{~s}$ of deposition and at a gap distance of $2.0 \mathrm{~cm} ; C$ ) Degree of alignment and voltage versus ground at the gap center versus flow rate ranging from 0.05 to 1 $\mathrm{ml} / \mathrm{h} ; \mathrm{D})$ SEM pictures of the samples spun with a flow rate of $0.05 \mathrm{ml} / \mathrm{h}$ and $1 \mathrm{ml} / \mathrm{h}$. 
The influence of flow rate was studied by keeping the gap distance at $2.0 \mathrm{~cm}$ while the flow rate ranged from $0.05 \mathrm{ml} / \mathrm{h}$ to $1 \mathrm{ml} / \mathrm{h}$. The results are presented in Figure 11C-D. At very low flow rates, e.g. $0.05 \mathrm{ml} / \mathrm{h}$, the alignment was nearly perfect but the packing of wires was poor (Figure 12A). The degree of alignment decreased with increasing flow rate. The electrical potential in the center of the gap was measured relative to the grounded electrodes to investigate the possible influence of residual electrostatic charges on fiber alignment. In principle, residual charges trapped in the fibers will flow to the collector electrode [13]. However, previous studies on alignment in electrospinning concluded that the electrical potential at the gap center plays a crucial role in the alignment process [13, 43]. I found that the electrical potential at the gap center increased with flow rate. Under steady state conditions, there should be a balance between charges arriving from the jet and charges flowing to the electrode. At higher flow rates, the flux of charges will also be higher. This may possibly cause charge buildup due to the low conductivity of the hybrid fibers, hindering the electrical discharge to the electrode. It was indeed observed that a high electrical potential at the gap center hindered fiber alignment. This observation supports previously reported simulations $[13,43]$, in which it was concluded that a near-zero potential at the gap center favors the lateral electromagnetic forces that drive fiber alignment.

Nanofiber alignment was limited to short deposition times $[7,16]$. I observed a decrease of alignment in the course of time. Figure 12A-B show this effect for a sample spun with a precursor flow rate of $0.25 \mathrm{ml} / \mathrm{h}$. The electrical potential at the gap center was monitored over time, but no increase of voltage at the center of the gap relative to the grounded electrodes over time was seen (Figure 12A). The loss of alignment probably occurred upon the formation of thicker layers of fibers. The bottom layer of fibers is thought to prevent new fibers from depositing on the electrodes, and hinder their discharge, so that the jet becomes unstable 
and starts whipping, leading to loss of alignment. An example is shown in Figure 12B, where the layers can be seen.

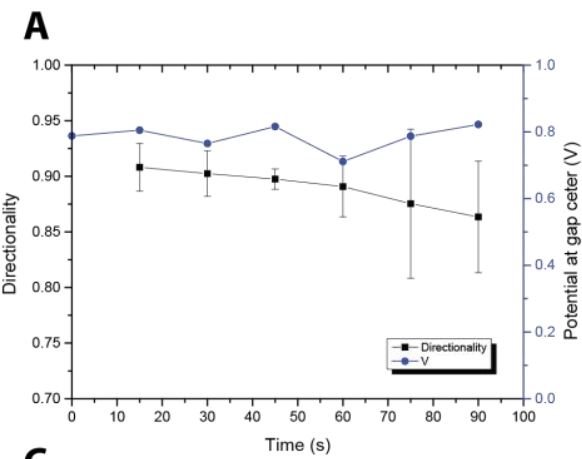

C

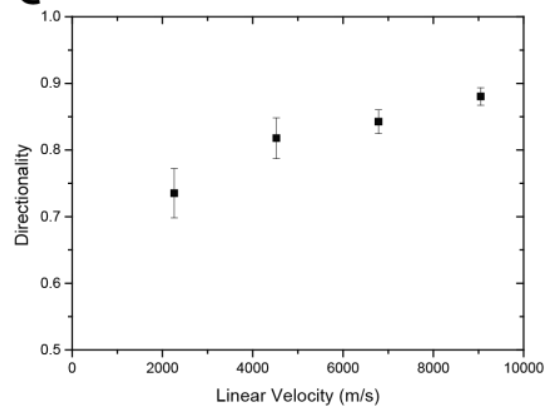

B
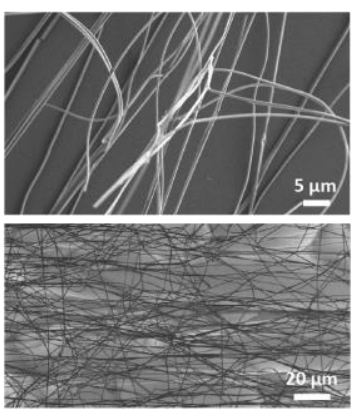

D

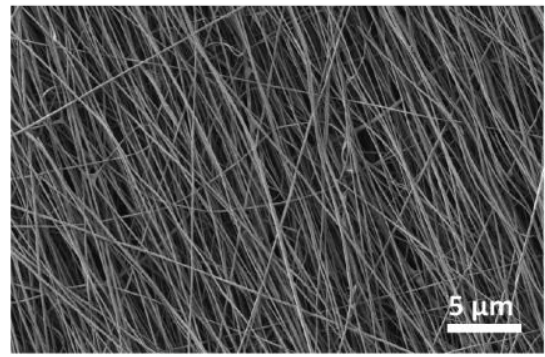

Figure 12. A) SEM pictures of the samples spun with a flow rate of $0.05 \mathrm{ml} / \mathrm{h}$ and $1 \mathrm{ml} / \mathrm{h}$. G-H). Influence of deposition time on directionality of a sample spun at $0.25 \mathrm{ml} / \mathrm{h}$ of $3 Y S Z$ precursor solution and a gap of $2.0 \mathrm{~cm} ; B$ ) Degree of alignment and voltage at the gap center versus time; $H$ ) SEM image of a samples spun for 90 s. C-D) Mechanical alignment of nanofibers with a rotating mandrel; C) Degree of alignment vs linear speed of the mandrel; D) Sample spun for $30 \mathrm{~min}$. All samples were spun at a flow rate of $1 \mathrm{ml} / \mathrm{h}$ of $3 Y S Z$ solution and thermally treated.

Mechanical alignment presents an alternative method to align nanofibers. I utilized a rotating mandrel as grounded electrode and a flow rate of $1 \mathrm{ml} / \mathrm{h}$. The rotating speed of the mandrel was varied and the influence on alignment was quantified. The degree of alignment vs. linear velocity of the mandrel is plotted in Figure $12 \mathrm{C}$. It can be seen that the alignment is better at higher speeds. This method allowed higher flow rates and longer deposition times to form thicker layers without influencing the alignment process negatively. Figure 12D shows a 
sample spun for $30 \mathrm{~min}$ at $1 \mathrm{ml} / \mathrm{h}$. However, the alignment achieved with the gap method, which can easily reach 90 to $95 \%$, is considerably better than the mechanical alignment, which is in the range of 70 to $90 \%$.

This study examines the processes involved in spinning, aligning fibers as well as forming hollow fibers in order to provide enough understanding and control. It can be an excellent complement to previous literature that examines the possibilities and formation of functional ceramic nanofibers without providing understanding in the processes involved $[1,2,18]$. 


\section{Conclusions}

This study shows the range of possibilities that electrospinning offers to fabricate dense and hollow ceramic fibers in a controlled manner. Using YSZ as an example compound, it has been shown that the fiber diameter can be varied from hundreds of nanometers to more than a micrometer. The upper and lower limits to the fiber diameter are mostly governed by the properties of the precursor solution used in the spinning process.

Electrospinning also allows a considerable degree of control over the fabrication of hollow fibers and arrays of aligned fibers. Limitations of the technique are, for instance, that the inner and outer diameter of the hollow fiber cannot be modified independently. Regarding aligned fibers, there is a limit to the number of fibers that can be aligned when it is electromagnetically driven, while the alignment is intrinsically poorer when it is mechanically driven.

Nevertheless, electrospinning has been proven to be a useful technique to produce ceramic nanofibers with control over their microstructure and properties. It offers a unique combination of control over fiber structure at relatively high production rates, which makes it a promising tool to produce dedicated nanofibers materials with unique properties. 


\section{References}

1. Li, D., J.T. McCann, Y. Xia, and M. Marquez, Electrospinning: A Simple and Versatile Technique for Producing Ceramic Nanofibers and Nanotubes. J. Am. Ceram. Soc., 2006. 89(6): p. 1861-1869.

2. Wu, H., W. Pan, D. Lin, and H. Li, Electrospinning of ceramic nanofibers: Fabrication, assembly and applications. Journal of Advanced Ceramics, 2012. 1(1): p. 2-23.

3. Kim, H.W., H.E. Kim, and J.C. Knowles, Production and Potential of Bioactive Glass Nanofibers as a Next-Generation Biomaterial. Advanced Functional Materials, 2006. 16(12): p. 1529-1535.

4. Rajesh Vasita, S.S.K., Nanofibers and their applications in tissue engineering. International Journal of Nanomedicine, 2008. 1(1): p. 15-30.

5. Pillay, V., C. Dott, Y.E. Choonara, C. Tyagi, L. Tomar, P. Kumar, L.C. du Toit, and V.M.K. Ndesendo, A Review of the Effect of Processing Variables on the Fabrication of Electrospun Nanofibers for Drug Delivery Applications. Journal of Nanomaterials, 2013. 2013: 22.

6. Reneker, D.H., A.L. Yarin, E. Zussman, and H. Xu, Electrospinning of Nanofibers from Polymer Solutions and Melts, in Advances in Applied Mechanics, A. Hassan and G. Erik van der, Editors. 2007, Elsevier. p. 43346.

7. Teo, W.E. and S. Ramakrishna, A review on electrospinning design and nanofibre assemblies. Nanotechnology, 2006. 17(14): R89.

8. Collins, G., J. Federici, Y. Imura, and L.H. Catalani, Charge generation, charge transport, and residual charge in the electrospinning of polymers: A review of issues and complications. J. Appl. Phys., 2012. 111(4): 044701.

9. Huang, Z.-M., Y.Z. Zhang, M. Kotaki, and S. Ramakrishna, A review on polymer nanofibers by electrospinning and their applications in nanocomposites. 2003. 63(15): p. 2223-2253.

10. Agarwal, S., A. Greiner, and J.H. Wendorff, Functional materials by electrospinning of polymers. Prog. Polym. Sci., 2013. 38(6): p. 963-991.

11. Reneker, D.H., A.L. Yarin, H. Fong, and S. Koombhongse, Bending instability of electrically charged liquid jets of polymer solutions in electrospinning. J. Appl. Phys., 2000. 87(9): p. 4531-4547. 
12. Yarin, A.L., S. Koombhongse, and D.H. Reneker, Taylor cone and jetting from liquid droplets in electrospinning of nanofibers. J. Appl. Phys., 2001. 90(9): p. 4836-4846.

13. Lihua, L. and A.D. Yuris, Analysis of the effects of the residual charge and gap size on electrospun nanofiber alignment in a gap method. Nanotechnology, 2008. 19(35): p. 355307.

14. Charge consequences in electrospun polyacrylonitrile (PAN) nanofibers. Polymer, 2005. 46(18): p. 7191.

15. Feng, J.J., The stretching of an electrified non-Newtonian jet: A model for electrospinning. Physics of Fluids (1994-present), 2002. 14(11): p. 39123926.

16. Li, D., Y. Wang, and Y. Xia, Electrospinning of Polymeric and Ceramic Nanofibers as Uniaxially Aligned Arrays. Nano Lett., 2003. 3(8): p. 11671171.

17. Ramaseshan, R., S. Sundarrajan, R. Jose, and S. Ramakrishna, Nanostructured ceramics by electrospinning. J. Appl. Phys., 2007. 102(11): 111101.

18. Dai, Y., W. Liu, E. Formo, Y. Sun, and Y. Xia, Ceramic nanofibers fabricated by electrospinning and their applications in catalysis, environmental science, and energy technology. Polym. Adv. Technol., 2011. 22(3): p. 326338.

19. Wu, Y.C., W.F. Koch, E. Arvay, D. Feng, L.A. Holland, and E. Juhasz, $A d c$ method for the absolute determination of conductivities of the primary standard $\mathrm{KCl}$ solutions from 0 -degrees- $\mathrm{C}$ to 50-degrees- $\mathrm{C}$. Journal of Research of the National Institute of Standards and Technology, 1994. 99(3).

20. Wu, Y.C., W.F. Koch, and K.W. Pratt, Proposed new electrolytic conductivity primary standards for $\mathrm{KCl}$ solutions. Journal of Research of the National Institute of Standards and Technology, 1991. 96(2): p. 11.

21. Pratt, K.W., W.F. Koch, Y.C. Wu, and P.A. Berezansky, Molality-based primary standards of electrolytic conductivity. Pure Appl. Chem., 2001. 73(11): p. 10.

22. Jacobs, V., R.D. Anandjiwala, and M. Maaza, The influence of electrospinning parameters on the structural morphology and diameter of electrospun nanofibers. J. Appl. Polym. Sci., 2010. 115(5): p. 3130-3136. 
23. Tan, S.H., R. Inai, M. Kotaki, and S. Ramakrishna, Systematic parameter study for ultra-fine fiber fabrication via electrospinning process. Polymer, 2005. 46(16): p. 6128-6134.

24. Pelofsky, A.H., Surface Tension-Viscosity Relation for Liquids. Journal of Chemical \& Engineering Data, 1966. 11(3): p. 394-397.

25. Schonhorn, H., Surface Tension-Viscosity Relationship for Liquids. Journal of Chemical \& Engineering Data, 1967. 12(4): p. 524-525.

26. Liu, Y., J.-H. He, J.-y. Yu, and H.-m. Zeng, Controlling numbers and sizes of beads in electrospun nanofibers. Polym. Int., 2008. 57(4): p. 632-636.

27. Brinker, C.J. and G.W. Scherer, Sol-gel science: the physics and chemistry of sol-gel processing. 1990: Gulf Professional Publishing.

28. Kiss, K., J. Magder, M.S. Vukasovich, and R.J. Lockhart, Ferroelectrics of Ultrafine Particle Size: I, Synthesis of Titanate Powders of Ultrafine Particle Size. J. Am. Ceram. Soc., 1966. 49(6): p. 291-295.

29. Stawski, T.M., S.A. Veldhuis, O.F. Göbel, J.E. Ten Elshof, and D.H.A. Blank, Effects of Reaction Medium on the Phase Synthesis and Particle Size Evolution of BaTiO3. J. Am. Ceram. Soc., 2010. 93(10): p. 3443-3448.

30. Valero, M., J. Tejedor, and L.J. Rodríguez, Encapsulation of nabumetone by means of -drug: (6-cyclodextrin)2:polyvinylpyrrolidone ternary complex formation. J. Lumin., 2007. 126(2): p. 297-302.

31. Theron, S.A., E. Zussman, and A.L. Yarin, Experimental investigation of the governing parameters in the electrospinning of polymer solutions. Polymer, 2004. 45(6): p. 2017-2030.

32. Shenoy, S.L., W.D. Bates, H.L. Frisch, and G.E. Wnek, Role of chain entanglements on fiber formation during electrospinning of polymer solutions: good solvent, non-specific polymer-polymer interaction limit. 2005. 46(10): p. 3372-3384.

33. Thompson, C.J., G.G. Chase, A.L. Yarin, and D.H. Reneker, Effects of parameters on nanofiber diameter determined from electrospinning model. Polymer, 2007. 48(23): p. 6913-6922.

34. Fridrikh, S., J. Yu, M. Brenner, and G. Rutledge, Controlling the Fiber Diameter during Electrospinning. Phys. Rev. Lett., 2003. 90(14): p. 144502.

35. Kappe, C.O., D. Dallinger, and S.S. Murphree, Practical Microwave Synthesis for Organic Chemists: Strategies, Instruments, and Protocols. Practical Microwave Synthesis for Organic Chemists: Strategies, Instruments, and Protocols. 2009. 1-299. 
36. Wang, J., J. Binner, B. Vaidhyanathan, N. Joomun, J. Kilner, G. Dimitrakis, and T.E. Cross, Evidence for the microwave effect during hybrid sintering. J. Am. Ceram. Soc., 2006. 89(6): p. 1977-1984.

37. Xie, Z., J. Yang, X. Huang, and Y. Huang, Microwave processing and properties of ceramics with different dielectric loss. J. Eur. Ceram. Soc., 1999. 19(3): p. 381-387.

38. Bhaskar, A., T.H. Chang, H.Y. Chang, and S.Y. Cheng, Low-temperature crystallization of sol-gel-derived lead zirconate titanate thin films using 2.45 GHz microwaves. Thin Solid Films, 2007. 515(5): p. 2891-2896.

39. Sundaresan, S., M. Rao, Y. Tian, J. Schreifels, M. Wood, K. Jones, and A. Davydov, Comparison of Solid-State Microwave Annealing with Conventional Furnace Annealing of Ion-Implanted SiC. J. Electron. Mater., 2007. 36(4): p. 324-331.

40. Sundaresan, S.G., M.V. Rao, Y.-I. Tian, M.C. Ridgway, J.A. Schreifels, and J.J. Kopanski, Ultrahigh-temperature microwave annealing of Al+- and P+implanted 4H-SiC. J. Appl. Phys., 2007. 101(7): 073708.

41. Goldstein, A., N. Travitzky, A. Singurindy, and M. Kravchik, Direct microwave sintering of yttria-stabilized zirconia at 2.45\&\#xa0;GHz. J. Eur. Ceram. Soc., 1999. 19(12): p. 2067-2072.

42. Wroe, R. and A.T. Rowley, Evidence for a non-thermal microwave effect in the sintering of partially stabilized zirconia. Journal of Materials Science, 1996. 31(8): p. 2019-2026.

43. Chaurey, V., P.-C. Chiang, C. Polanco, Y.-H. Su, C.-F. Chou, and N.S. Swami, Interplay of Electrical Forces for Alignment of Sub-100 nm Electrospun Nanofibers on Insulator Gap Collectors. Langmuir, 2010. 26(24): p. 1902219026. 


\section{Chapter 3}

\section{Flexible Yttrium-Stabilized Zirconia Nanofibers Offer Bioactive Cues for Osteogenic Differentiation of Human Mesenchymal Stromal Cells}

\section{Abstract}

Currently, the main drawback of ceramic scaffolds used in hard tissue regeneration is their brittleness. Stabilized zirconia, especially the tetragonal $3 \%$ yttrium-stabilized zirconia (YSZ) phase, has been considered as a bioinert ceramic material with high mechanical strength. In the present work, flexible nanofibrous YSZ scaffolds were prepared by electrospinning. The obtained scaffolds showed remarkable flexibility at the macroscopic scale, while retaining their stiffness at the microscopic scale. The surface nanoroughness of the scaffolds could be tailored by varying the heat treatment methods. My results demonstrate the osteogenic differentiation and mineralization of seeded human mesenchymal stromal cells (hMSCs) were supported by the nanofibrous YSZ scaffolds, in contrast to the well-known bioinert behavior of bulk YSZ. These findings highlight that flexible ceramic scaffolds are an appealing alternative to the current brittle ceramics for bone tissue regeneration applications. 


\section{Introduction}

Bioceramics have been widely used for several decades to repair bone damage [13], since they are promising materials due to their similarity to bone in terms of chemical properties [4-7]. Within the area of bioceramics, a clear distinction is made between bioinert and bioactive materials. Bioinert ceramics can be defined as those that do not bond with the surrounding tissues, nor induce a cell response. Examples of bioinert materials are alumina and yttria-stabilized zirconia (YSZ) [2, 3]. Conversely, bioactive ceramics are materials that can actively induce a specific cell response (e.g. cell differentiation) and, in some cases, are resorbable. These materials include hydroxyapatite (HA), 6 -calcium phosphates and bioactive glasses [1-3]. One of the major limitations of ceramic scaffolds for hard tissue regeneration is that they suffer from brittleness, which may facilitate fragile failure when implanted $[4,6,8]$. Among the aforementioned bioceramic materials, zirconia has the highest mechanical strength, especially the $3 \%$ yttriumstabilized tetragonal zirconia (YSZ) phase $[9,10]$.

The scaffold's microstructure also plays a crucial role. Microporosity provides a large surface area for cell adhesion, thus improving the biological response [11]. However, the scaffolds' mechanical properties are negatively affected [4, 7]. Porosity reduces the mechanical strength of materials [7]. Tailor-made design of the nanoarchitecture of porous biomaterials may help to overcome these issues [9, 12-14]. A recent study demonstrated a nanoarchitectured aluminosilicate ceramic with improved ductility and flexible recovery after compressive stresses [12]. Nanofiber mats of titania, silica and zirconia with enhanced flexibility have also been reported [13-20].

To fabricate scaffolds with fiber dimensions on the nano- to microscale mimicking the features of the natural extracellular matrix (ECM), electrospinning has been 
used broadly to fabricate scaffolds for regenerative medicine [21-23]. Cells do not only respond to chemical and physical stimuli from their neighboring cells, but also from the surrounding fluid and ECMs [24]. The characterization and control of surface properties, such as surface roughness, can therefore be used to optimize cell attachment, proliferation and differentiation.

In this article, I propose the fabrication of a flexible YSZ nanofiber material for bone tissue engineering. Recently, YSZ-based fibers have been fabricated by electrospinning for various application, such as fuel cells [25] and filtration [18], but their use in the biological and biomedical field has not been explored to my knowledge. Herein, I introduce flexible YSZ ceramic nanofibers in the field of regenerative medicine. The YSZ fibers typically have lengths of the order of meters, instead of just a few millimetres as reported earlier [25], and a high gravimetric density. The sintering temperature that I employed to densify the ceramic nanofibers was much lower than in earlier studies [25], so that sintering between fibers was avoided, which contributed further to their flexibility and retention of mechanical properties upon cyclic deformation. Indeed, these YSZ ceramic nanofibers have remarkable flexibility compared to the traditional bioactive ceramics and their surface nanoroughnesses can be tailored for cellular response. Interestingly, in contrast to the well-known bioinert behavior of bulk YSZ, nanofiber meshes support in vitro osteogenic differentiation and mineralization of human mesenchymal stromal cells (hMSCs). This is the first study showing that flexible YSZ ceramic fibers with tailored surface nanoroughness can conduct osteogenic differentiation of hMSCs. The flexibility of the scaffold helps to overcome the problem of the fragile fracture that is characteristic for traditional bioceramic scaffolds. Therefore, it provides a complement to the currently available library of bioactive ceramic materials for bone tissue regeneration. 


\section{Results and discussion}

The ceramic nanofibers were fabricated by electrospinning with a mixture of a YSZ precursor and polyvinylpyrrolidone (PVP). Subsequently, the resulting fiber mats were annealed by means of conventional or microwave-assisted heating in static air at $850{ }^{\circ} \mathrm{C}$ for $2 \mathrm{~h}$ using different heating rates (i.e. to burn out the organic phases and for crystalline phase formation). The obtained nanofibers had a diameter of $530 \pm 120 \mathrm{~nm}$ (Figure S1), and the fiber mats had a thickness of approximately $120 \mu \mathrm{m}$. A scanning electron microscopy (SEM) image of the material is shown in Figure 1A. The YSZ nanofiber mats exhibited extraordinary flexibility not often demonstrated for pure ceramic materials. It behaved as a paper-like material (Figure 1B). The material could achieve total deflection (180 bending) without mechanical failure. Moreover, the deflection could be cycled at least 1000 times without breaking the material when a 3-point bending test was performed. To the best of my knowledge, the fabrication of ceramic nanofiber mats with such high flexibility and good fatigue behavior has not been reported until now. The flexibility of electrospun silica-based mats has been attributed to the amorphous nature of the material, which is therefore more robust than crystalline materials $[14,26]$. However, that hypothesis does not explain the behavior of the crystalline YSZ nanofibers reported here (X-ray diffractograms showing crystalline nature are shown in Figure S2). Park et al. hypothesized that the flexibility of crystalline $\mathrm{TiO}_{2}$ was the result of the physical entanglement as single nanofibers are brittle [13]. However, I observed that also single nanofibers were able to withstand a high degree of deflection. Probably due to the long length of the nanofibers, the mechanical energy could be dissipated under the elastic deflection. The coherence of fibers mats composed of long fibers is better than that of fiber mats composed of an assembly of fiber fragments. Moreover, the sintering temperature of $850{ }^{\circ} \mathrm{C}$ that I employed is much lower than in earlier 
reports on YSZ nanofibers where a temperature of $1500{ }^{\circ} \mathrm{C}$ was used [25]. As a result, my YSZ fibers densified but did not sinter together, which contributes to their freedom of movement and thus to their flexibility, and makes them less susceptible to fatigue-like phenomena. The flexibility of the mat was further quantified by microindentation tests. The Young's modulus of the material was $1.11 \pm 0.24 \mathrm{MPa}$, more than 5 orders of magnitude lower than that of bulk YSZ (Figure 1C), which is in the range of $178-222 \mathrm{GPa}$ according to literature data [9, 27-29].I also measured the mechanical properties of single nanofibers by a 3-point bending test using atomic force microscope (AFM) (Figure 1D). The measured apparent Young's modulus of a single fiber was $30 \pm 3 \mathrm{GPa}$, and a high deflection angle could be achieved. The apparent Young's modulus of single fibers was several orders of magnitude higher than the macroscopic mat, but still a factor of 7 lower than bulk YSZ (Figure 1C). This result is in accordance with other studies on electrospun ceramic nanofibers, which report similar decreases in Young's modulus when measured via a three point bending test using AFM [30, 31]. The diameter and mechanical properties of the nanofibers at the different length scales did not vary significantly with the annealing treatment (Figure S3).

A special set-up was designed to perform a 3-point bending fatigue test on the mat (Figure1E). The experiment consisted of 200 cycles of $45^{\circ}$ deflection, while the applied force was monitored (Figure 1F). The sample did not break, and to my surprise I observed a slight increase of the force necessary to deflect the sample. Chen et al. reported slipping of individual nanofibers under tensile stress [18]. It may be possible that such a phenomenon occurs during deflection, gradually leading to a rearrangement of the fibers with an increasing number of cycles, ultimately resulting in a slightly stiffer material. 

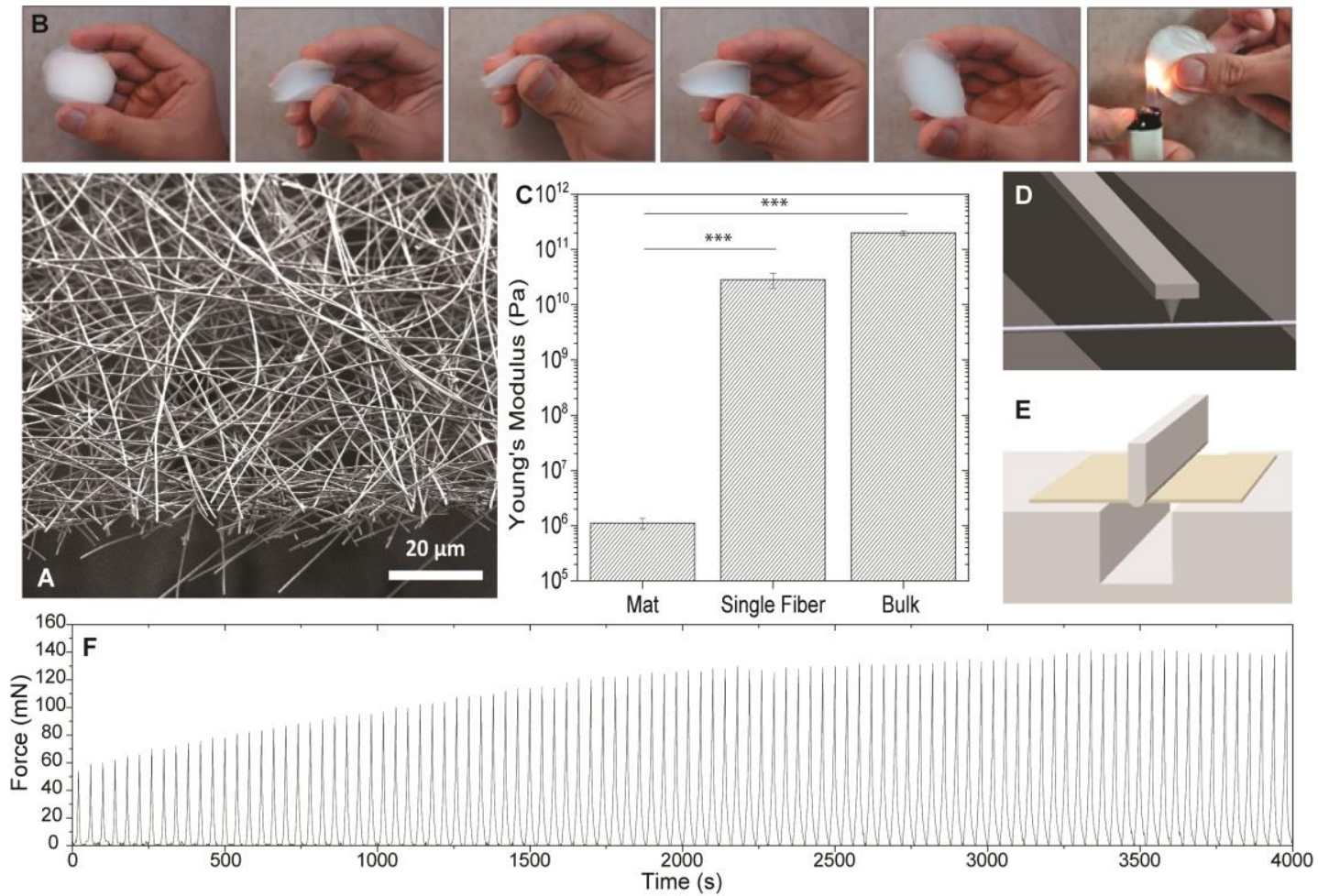

Figure 1. The flexibility of YSZ nanofiber mats. (A) SEM images of a YSZ nanofiber mat, Scale bar is $20 \mu \mathrm{m}$. (B) Pictures of the YSZ mat showing its flexibility and fire resistance ceramic nature. (C) Mechanical properties of a nanofiber mat by microindentation (at macroscopic scale), a single nanofiber by 3 point bending using AFM (at microscopic scale), and bulk YSZ (taken from literature ${ }^{27-29}$ ). (D) Schematic overview of a 3 point bending test on a single nanofiber using AFM. (E) Schematic illustration of a 3 point bending fatigue test on the nanofiber mat. (F) Representative histogram of 200 cycles of the 3 point bending fatigue test on a YSZ nanofiber mat in which the force was monitored as a function of time. Error bars represent standard deviation of the mean. The statistical significance was determined using a one way ANOVA test, where $*^{* *} p<$ 0.001 .

Moreover, the nanofibers mat offered a very low density, in the range of 0.06 $0.09 \mathrm{~g} / \mathrm{cm}^{3}$. Based on SEM observations, i.e. the absence of pores and grain boundaries, each individual fiber can be considered as nearly dense. The Young's modulus of the nanofiber mats and individual fibers were plotted vs. the density as shown in Figure 2. For comparison, the values of other traditional materials 
were also plotted. It can be seen that the ceramic nanofibers mat behaved like a foam in terms of stiffness and density. This means that it is lighter and more flexible than other polymers, ceramics and porous ceramics. Nevertheless, individual nanofibers offer stiffness values close to ceramics and typical of porous ceramics or metals, 5 orders of magnitude stiffer than the mat.

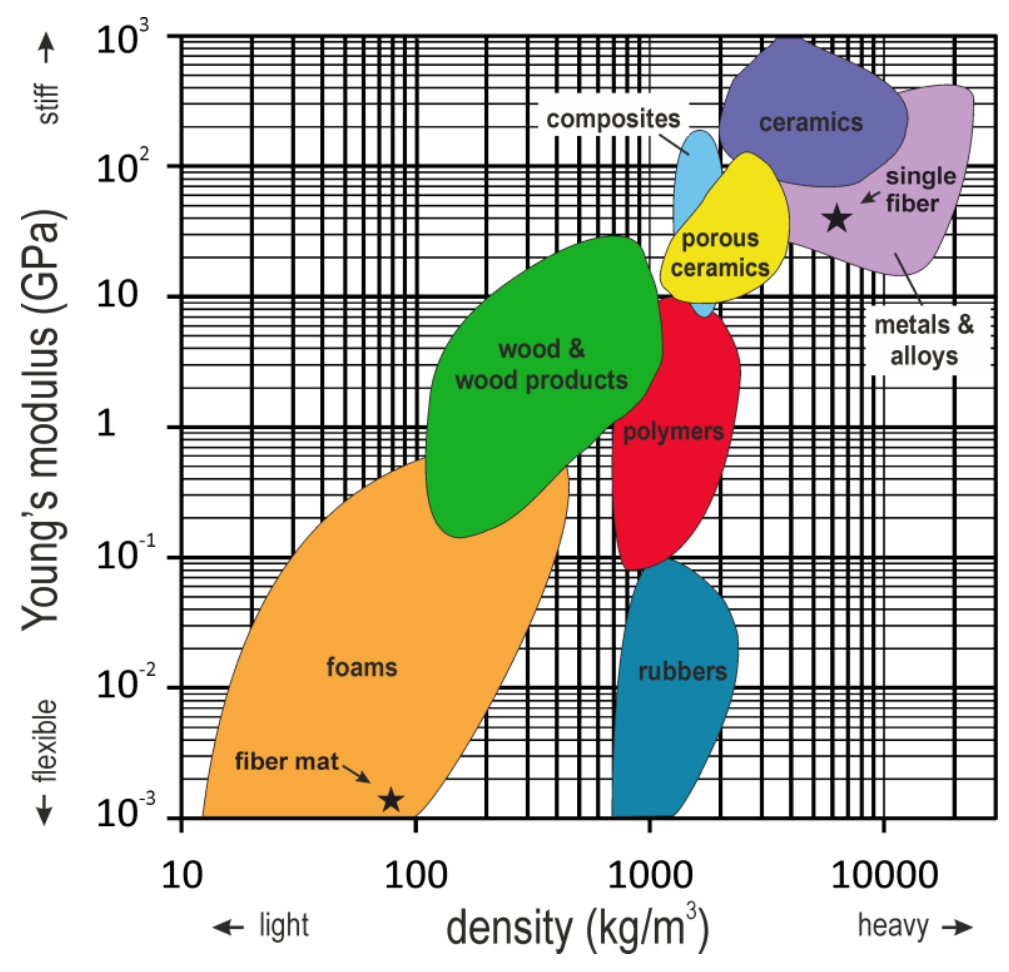

Figure 2. Plot of Young's Modulus vs density. The nanofiber mat and single nanofiber are compared with other traditional materials.

The surface morphology and grain size of the fibers were modified by annealing the samples under different conditions. The green fiber mats were annealed in a conventional oven (CO) at 1 or $5{ }^{\circ} \mathrm{C} / \mathrm{min}$ heating rate and, in a microwave oven (MW) at $5^{\circ} \mathrm{C} / \mathrm{min}$ or by rapid thermal annealing (i.e. thermal shock). Rapid thermal annealing (RTA) is a method in which the $\mathrm{MW}$ is preheated to $850^{\circ} \mathrm{C}$ prior to placing the sample in the oven. The samples annealed in the $\mathrm{CO}$ showed rougher 
surface morphologies than the samples annealed in the MW even at the same heating rate, as shown by SEM (Figure $3 \mathrm{~A}$ ) and AFM (Figure 3B) analysis. This was further confirmed by the statistical analysis performed on the roughness data (Figure 3C). However, varying the heating rate did not result in significant differences among samples that were annealed by the same heating mechanism. The samples annealed in the $\mathrm{CO}$ at $1{ }^{\circ} \mathrm{C} / \mathrm{min}$ and $5{ }^{\circ} \mathrm{C} / \mathrm{min}$ had roughnesses of 0.8 $\pm 0.3 \mathrm{~nm}$ and $0.7 \pm 0.2 \mathrm{~nm}$, respectively. Both samples annealed in the MW had a roughness of $0.4 \pm 0.1 \mathrm{~nm}$. The heating mechanism had a significant influence on surface roughness. Samples annealed at $5{ }^{\circ} \mathrm{C} / \mathrm{min}$ in a $\mathrm{CO}$ had a rougher surface than the samples annealed in a MW at the same heating rate $(0.7 \pm 0.2 \mathrm{~nm}$ vs 0.4 $\pm 0.1 \mathrm{~nm}$ ). These results were consistent with the surface morphologies in SEM images (Figure 3A). However, it is noted that the roughness with AFM is measured in the out-of-plane direction, whereas the SEM images shows in-plane features of surface morphology. Different grain sizes were observed for each heating rate (Figure 3D) as calculated from the full width at half maximum (FWHM) in the XRD patterns (Figure S2). Samples annealed in the $\mathrm{CO}$ at $1{ }^{\circ} \mathrm{C} / \mathrm{min}$ had the largest grain size $(24 \pm 1 \mathrm{~nm})$. Samples annealed in the $\mathrm{CO}$ and $\mathrm{MW}$ at $5{ }^{\circ} \mathrm{C} / \mathrm{min}$ had intermediate grain sizes of $22 \pm 1 \mathrm{~nm}$ and $18 \pm 1 \mathrm{~nm}$, respectively. The sample annealed in the MW using RTA had a much smaller grain size of $9.5 \pm 0.1 \mathrm{~nm}$. The samples annealed using lower heating rates showed larger grain sizes, irrespective of the heating mechanism. This can be attributed to the longer effective sintering times at high temperature under such conditions. The heating mechanism also affected the grain size. The samples annealed at $5^{\circ} \mathrm{C} / \mathrm{min}$ in a $\mathrm{CO}$ had a larger grain size than the samples annealed in a MW at the same heating rate. YSZ has previously been reported to exhibit enhanced sintering behavior under microwave radiation heating [32,33]. Xie et al. reported smaller and more uniform grain sizes when YSZ was sintered in a MW, which is in accordance with my data (Figure 3D) [33]. The YSZ nanofibers presented here have a different 
surface nanostructure than any previously reported YSZ topography. They are nearly 1-dimensional objects in a porous matrix, which affects the heat transport and crystallization rates. Nevertheless, the smoothening of the surface, when annealed using a MW, can be attributed to a step-bunching effect (i.e. aggregation of the ceramic steps) [34], sudden shrinkage and densification [32], and/or improved oxygen transport [35].

To elucidate the performance of my nanofibrillar YSZ in bone tissue regeneration, hMSCs were grown on samples annealed at $5{ }^{\circ} \mathrm{C} / \mathrm{min}$ using both $\mathrm{CO}$ and $\mathrm{MW}$ heating. I chose these samples as they have the same temperature profile during annealing, grain sizes in the same order of magnitude and similar fiber diameter distributions, yet different surface roughness. Recent studies revealed the importance of nanoscale and submicron-sized topographic cues to modulate fundamental cell behavior, including proliferation, migration, and differentiation [36-38]. I hypothesized, therefore, that the nanofibrous structure and the different surface roughness of YSZ scaffolds may confer bioactive cues to an otherwise biologically inert ceramic material. Such topographical cues would ultimately result in varying cell morphology and, consequently, in different cell activities. The bioactivity of hMSCs on ceramic scaffolds was investigated by monitoring the cell viability, cellular morphology, cellular metabolism, alkaline phosphatase (ALP) activity, and gene expression in basic culture medium (BM) and osteogenic culture medium (OM) at days 7 and 14. Polystyrene tissue culture plates were used as a positive control (data not shown). The production of mineralized matrix was investigated after culturing cells-scaffold constructs in mineralization culture medium (MM) up to 28 days. Here, different culture media were chosen to investigate the influence of YSZ scaffolds on the osteogenesis potential of hMSCs in the presence or absence of osteoinducting soluble factors. $\mathrm{BM}$ is a maintenance medium that does not trigger the osteogenic differentiation 
of hMSCs [39-40]. On the other hand, OM induces osteogenic differentiation of hMSCs, whereas MM supports osteogenic differentiation and promotes the formation of mineralized matrix $[39,41]$.

A
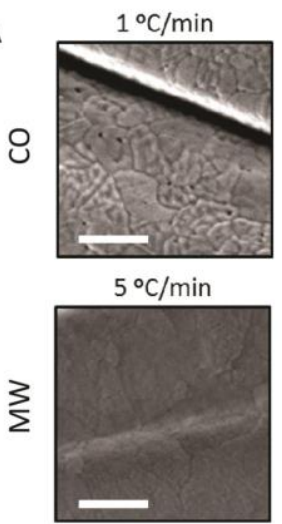

C

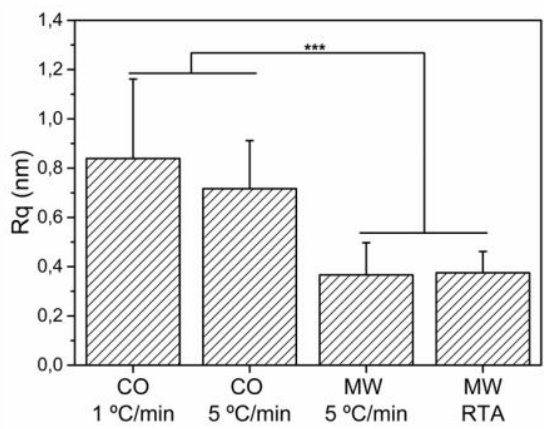

RTA

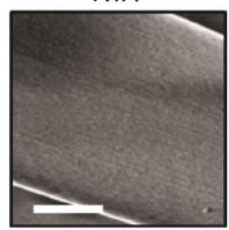

Scale bar $=200 \mathrm{~nm}$
B

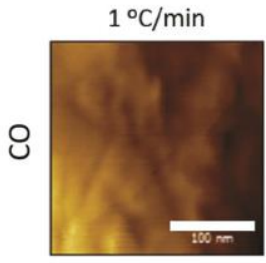

D
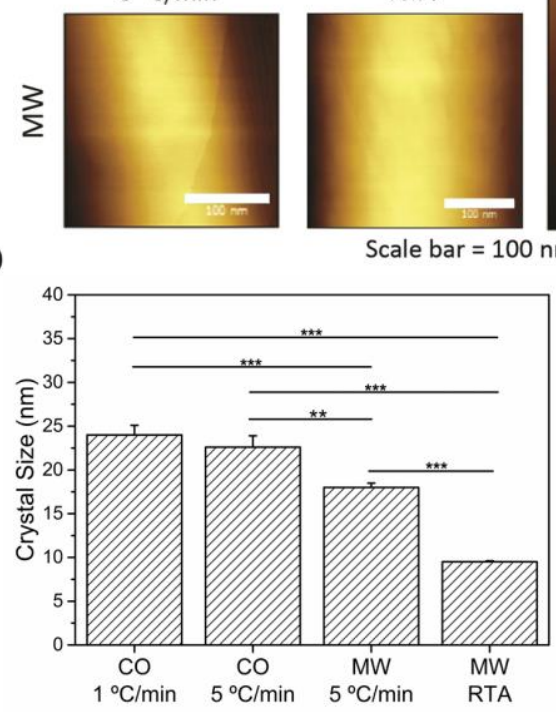

Figure 3. The effect of annealing procedure on the fiber surface morphology. (A) SEM images of YSZ nanofibers, the scale bar is $200 \mathrm{~nm}$. (B) AFM images of YSZ nanofibers, the scale bar is $100 \mathrm{~nm}$. (C) Roughness data extracted from AFM scans. (D) Grain sizes of the fibers calculated from the FWHM of the (111) peak of the XRD patterns. Error bars represent standard deviation of the mean. The statistical significance was determined using a one way ANOVA test, where ${ }^{* *} p<0.01, * * * p<$ 0.001 .

To evaluate the viability of hMSCs on YSZ nanofibrillar scaffolds, a Live/Dead assay ${ }^{\bullet}$ was performed after 3 days of culture. The fluorescence microscopy images of both live and dead cells on scaffold are shown in Figure S4. All investigated 
scaffolds showed a high rate of viable cells, which indicates no cytotoxic effects derived from the developed YSZ nanofibrillar scaffolds.

The cell morphology on YSZ scaffolds was investigated by SEM (Figure 4A-C). The cell cytoskeleton was further assessed by staining with phalloidin for actin filaments (Figure 4D-F), and the average surface area for single cell was quantified (Figure 4G). Cells on CO annealed scaffolds had a higher cell area compared to MW annealed scaffolds, which could be correlated to the difference in surface roughness of the nanofibers. When comparing to that on bulk YSZ (having a roughness of $19 \pm 0.94 \mathrm{~nm}$ which is typical for a smooth bulk ceramic surface), no significant difference in the average cell area was found possibly due to the combined effect of surface roughness and nanoscale fibrous structure. It is worthy to mention that the difference in surface roughness between YSZ nanofibers and bulk YSZ illustrates that the outer dimensions of fibers and bulk material differ, but also their surface morphologies.

Cell metabolic activity, which is indirectly related to cell viability, was determined using a PrestoBlue assay. The metabolic activity of hMSCs showed a similar profile in BM and OM (Figure S5). Bulk YSZ scaffolds had a significantly higher metabolic activity than all nanofibrous scaffolds, which could be due to the higher differentiation of hMSCs on nanofibrous scaffolds compared to bulk YSZ. Similar phenomena have been reported previously by Yuejun and his colleagues [42]. They found that hMSCs had higher metabolic activity on stiffer PDMS than the soft one while a higher differentiation activity of hMSCs were observed on soft PDMS compared to stiffer one. In addition, no significantly different metabolic activity was observed between $\mathrm{CO}$ and MW annealed scaffolds. This indicates that the surface roughness differences did not affect the cellular metabolism in the present work. 

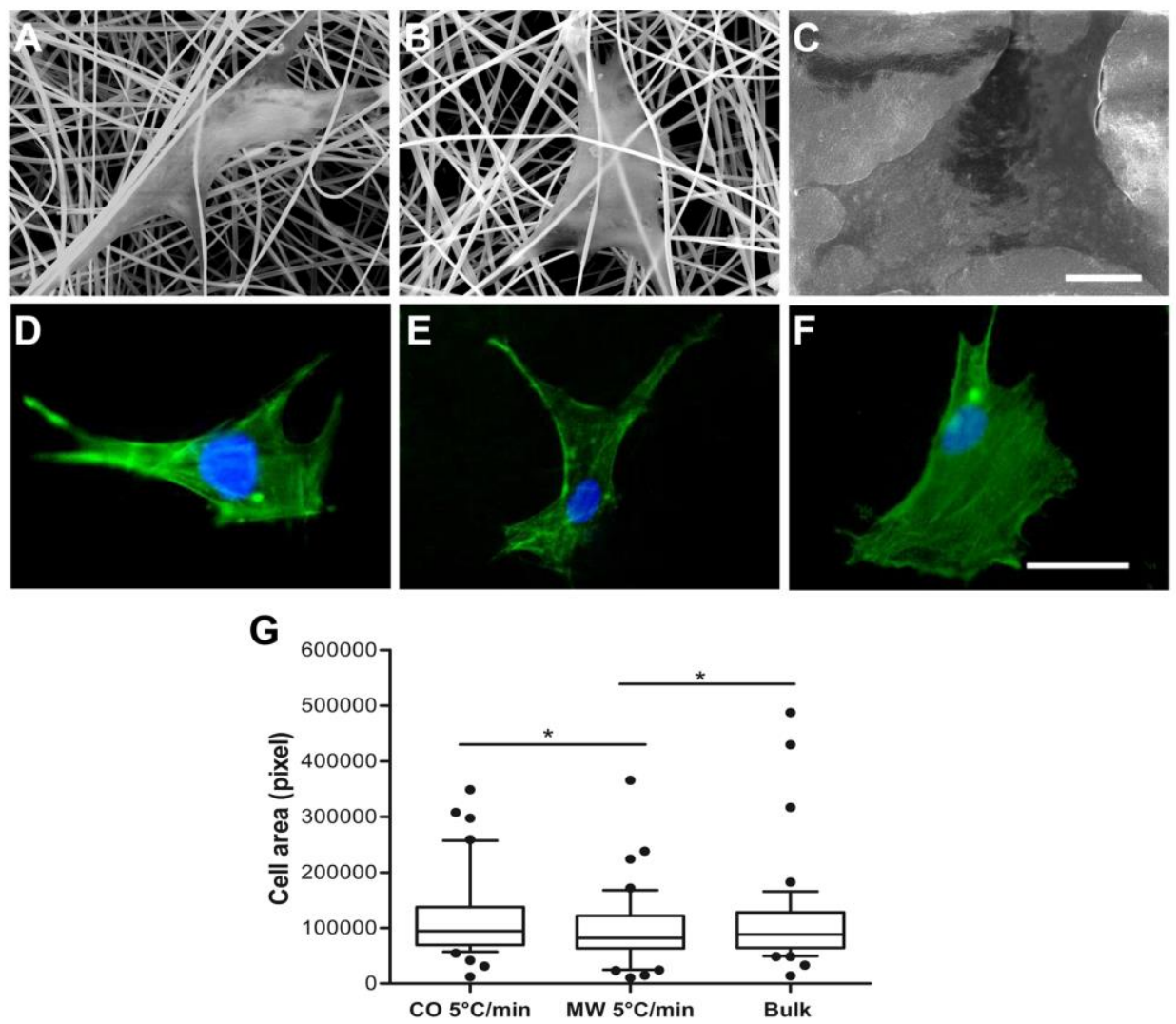

Figure 4. Characterization of cell morphology and actin cytoskeleton organization of hMSCs after 3 days of culture in BM. Representative SEM images of hMSCS on (A) microwave annealed (MW $5^{\circ} \mathrm{C} / \mathrm{min}$ ) nanofiber scaffolds, (B) conventional oven annealed (CO $5^{\circ} \mathrm{C} / \mathrm{min}$ ) nanofiber scaffolds, and (C) YSZ bulk disk scaffolds. Representative images of actin cytoskeleton organization of hMSCs on (D) microwave annealed ( $\mathrm{MW} 5^{\circ} \mathrm{C} / \mathrm{min}$ ) scaffolds, (E) conventional oven annealed $\left(\mathrm{CO} 5^{\circ} \mathrm{C} / \mathrm{min}\right.$ ) scaffolds, and (F) YSZ bulk disk scaffolds (actin filaments labeled with phalloidin are shown in green and nuclei labeled with DAPI are shown in blue). (G) Quantification of projected cell area on the investigated scaffolds. The statistical analysis was determined using two-tailed Student's t-test and ${ }^{*} p<0.05$ was considered as significant difference. The scale bar in $(A-C)$ and $(D-F)$ are10 $\mu \mathrm{m}$ and $50 \mu \mathrm{m}$ respectively.

Polymerase chain reaction (PCR) was used to quantify the expression of a panel of osteogenic genes (Figure 5C and Figure S6). Gene expression of Runt-related transcription factor 2 (RUNX2) was significantly higher for CO and MW annealed 
scaffolds compared to bulk YSZ in both culture media. RUNX2 is considered as a focal point for integration of a variety of signals affecting the differentiation process of hMSCs into osteogenesis in the early stage, because it stimulates other downstream osteo-related genes such as osteopontin (OPN), osteocalcin (OCN ), bone sialoprotein (BSP), and type I collagen [43]. Collagen type I alpha 1 (COL1A1) is fundamental for the development of the bone cell phenotype. It is actively expressed in the first proliferation period and then gradually down-regulated during subsequent osteoblast differentiation [44]. hMSCs cultured on annealed nanofibrous scaffolds showed higher expression of COL1A1 compared to bulk YSZ disks in both culture media. This is also associated with a significantly higher upregulation of OCN and BSP on the nanofibrillar scaffolds in BM compared with bulk YSZ disks (Figure S6). Furthermore, the rougher meshes supported further enhanced OCN and BSP gene expression than the smoother ones, thus suggesting the possible role of surface topography on inducing hMSCs osteogenic differentiation, as also supported by other studies [45, 46]. OPN was only significantly up-regulated on nanofibrillar scaffolds at day 7 in OM, while no beneficial effect of surface topography or nanofibrillar cues were observed for OCN or BSP in OM both at day 7 and at day 14. Since one of the route for hMSCS differentiation towards the osteogenic lineage passes through endochondral development $[47,48]$, the down-regulation of OCN and BSP on nanofibrous scaffolds in comparison with their bulk disks could be also attributed to a higher hypertrophic differentiation of hMSCs, and therefore a less mature osteogenic differentiation, on bulk disks due to their smooth surface properties in combination of medium stimuli. The smooth surface properties will affect the cellto-material adhesion force and could have consequently influenced cell fate as well [49]. I also observed the expression of bone morphogenetic protein 2 (BMP2), which is known to be stimulating hMSCs along the osteogenic lineage [50]. BMP2 had a higher expression in all tested media on both nanofibrillar 
scaffolds compared to bulk YSZ except for day 7 in OM. Altogether, the ceramic nanofibrous scaffolds supported the osteogenic gene expression in both BM and $\mathrm{OM}$, thus suggesting a possible role of the nanofibrillar cues inherently built in the YSZ meshes in contributing to hMSCs osteogenic differentiation. In addition, the varying surface roughness resulting from the different fiber annealing processes showed a net beneficial effect in case of OCN and BSP genes, which occurred only for the rougher nanofibrous scaffolds obtained by employing conventional heating. It is also worth to mention that there were different trends in $\mathrm{OM}$ compared to BM, which might suggest that the differentiation of hMSCs in the present study was not only due to surface roughness but also other physical stimuli cues such as surface topography, porosity and physical cues associated to the nanoscale nature of the fibrillar meshes. Further studies should aim at clarifying the specific role of each of these material properties on cell differentiation, and the consequent osteoinductive nature of such nanofibrillar scaffolds, in addition to the osteoconductive properties here observed, both in vitro and in vivo. 
A

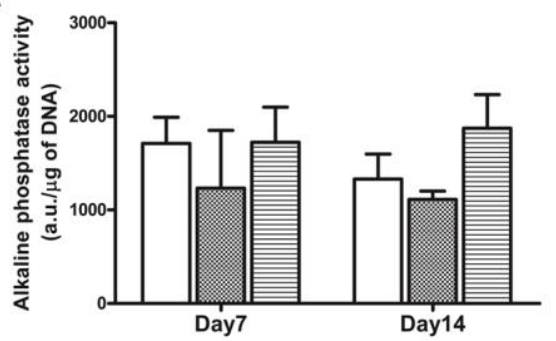

B

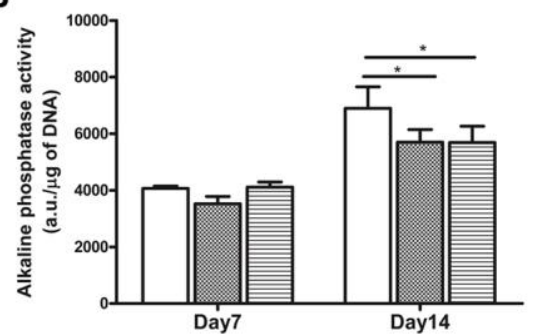

C
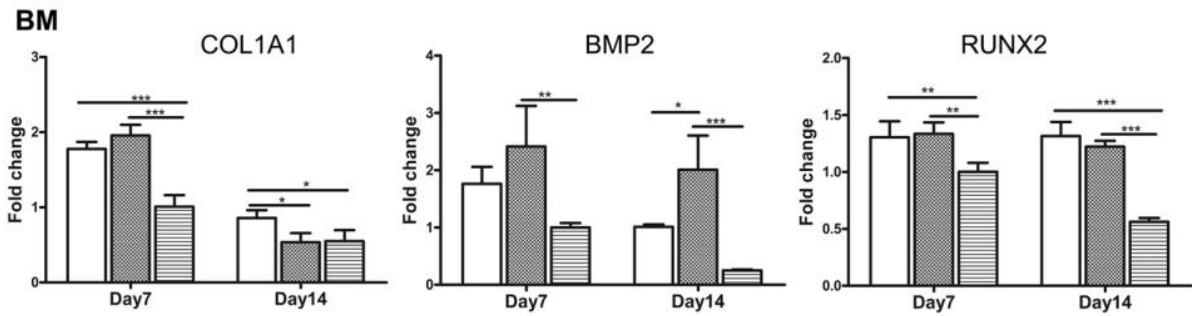

RUNX2
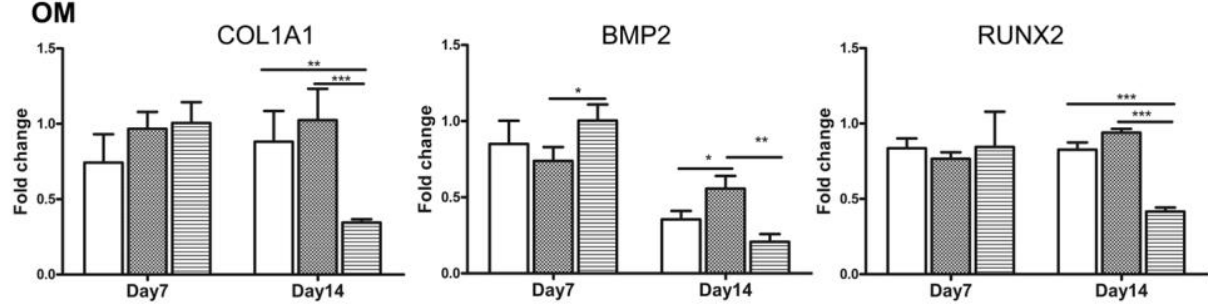

$\mathrm{CO} 5^{\circ} \mathrm{C} / \mathrm{min}$

的 $5^{\circ} \mathrm{C} / \mathrm{min}$

目 Bulk

Figure 5. ALP activity (A and B) and gene expression analysis (C) from hMSCs cultured on conventional oven annealed $\left(\mathrm{CO}^{\circ} \mathrm{C} / \mathrm{min}\right)$, microwave annealed $\left(\mathrm{MW} 5^{\circ} \mathrm{C} / \mathrm{min}\right)$ and $\mathrm{YSZ}$ bulk disk scaffolds. ALP activity of hMSCs cultured in BM (A) and OM (B) at day 7 and 14 (the final ALP activity level was shown after normalizing to DNA amount). (C) Gene expression analysis for COL1A1, BMP2, and RUNX2 at day 7 and 14 in BM and OM (the final gene expression level was presented after normalizing to day 7 bulk YSZ). Error bars represent standard deviation of the mean. ${ }^{*} p<0.05,{ }^{* *} p<0.01,{ }^{* * *} p<0.001$.

At a protein level, the up-regulation of ALP activity is a common marker considered for the early stage of osteogenesis [43]. In BM, no significant differences were observed in ALP activity (Figure 5A). On the other hand, the ALP activity increased between day 7 and day 14 in OM and was significantly enhanced for $\mathrm{CO}$ annealed scaffolds compared to both MW annealed scaffolds and bulk YSZ (Figure 5B). These results indicate that the topographical properties 
of nanofibers, including larger surface area, higher porosity, and fibrous morphology, play a positive role in the osteogenic process of hMSCs. Similar results to my findings have been reported by Hae-Won Kim and colleagues [51] who used bone marrow-derived stem cells to investigate the osteogenic potential of newly developed glass fibers. Their results demonstrated that bioactive glass nanofibers showed a stronger ALP staining with respect to glass disks.

The ultimate deposition of a calcium-containing bone-like mineralized matrix is an index of extended osteogenic maturation of hMSCs. Mineralization was stained with alizarin red S after 28 days of culture (Figure 6 and Figure S7). When hMSCs were cultured on scaffolds in BM, little mineralized ECM was produced and no apparent differences were observed among the different scaffolds. In MM, a robust mineralized matrix was observed on bulk YSZ discs, while a strikingly significant increase in the amount of mineralized matrix was observed on the nanofibrous scaffolds. Quantification of the calcium deposits corroborated the staining results, showing significantly enhanced calcium deposition on both fiber scaffolds compared to the bulk YSZ discs (Figure 6B). Here, the rougher CO annealed scaffolds resulted in a further enhancement of calcium content compared to the MW annealed scaffolds. This result is similar to the finding by Takeuchi and his co-workers, who demonstrated that osteoblast show a higher rate of matrix mineralization on rougher titanium surfaces [52]. A recent study showed that hMSCs cultured on calcium phosphate cements had a mineral concentration of about $1 \mathrm{mM}$ per scaffold at day 21 [53]. Compared to this study, my nanofibers annealed in a conventional oven had almost 3 folds higher mineral concentration. However, when comparing to other studies, care should be taken as different cell donors, time point and cell seeding density have been used. 

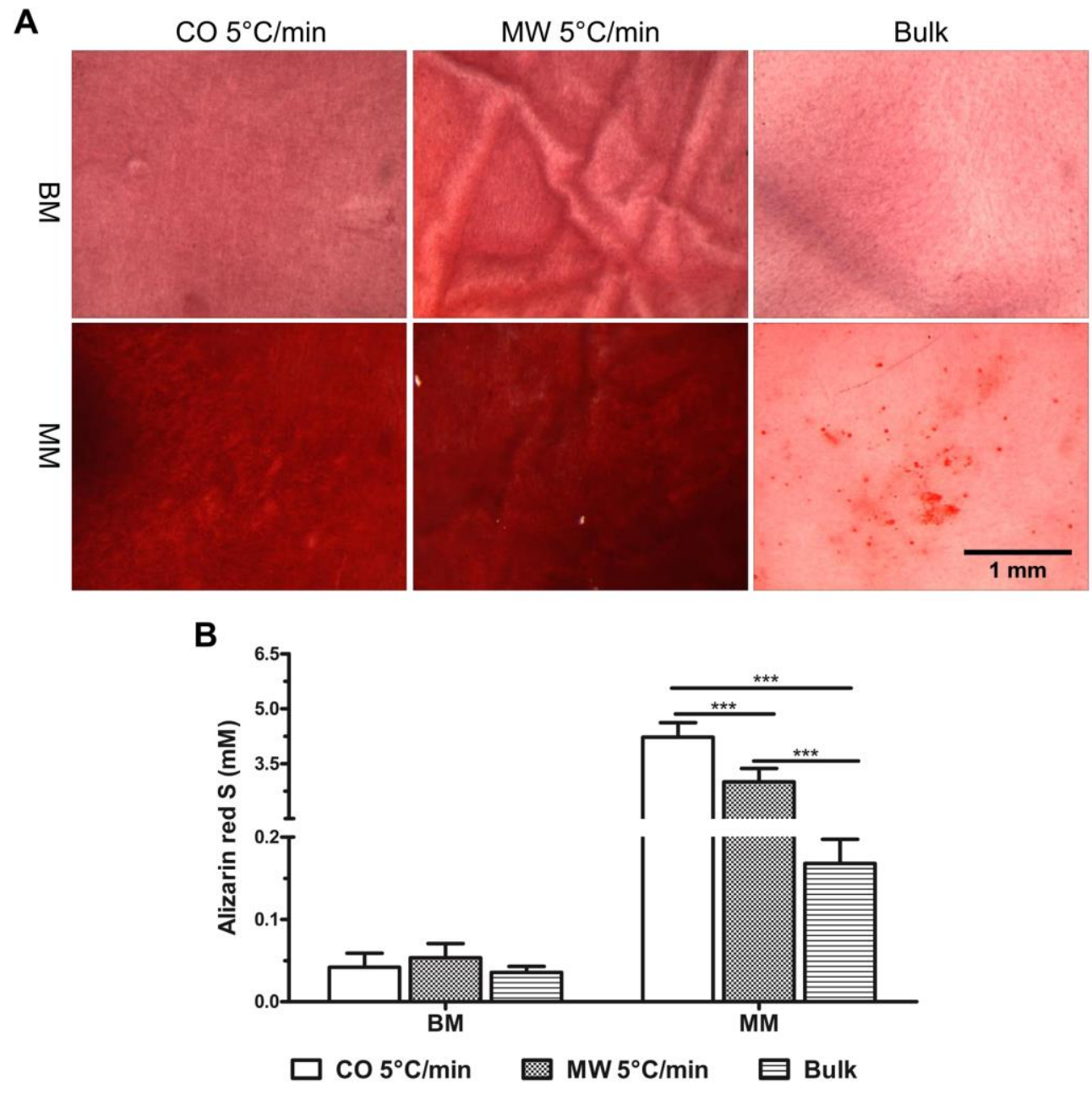

Figure 6. Calcium mineralization on conventional oven annealed (CO $\left.5^{\circ} \mathrm{C} / \mathrm{min}\right)$, microwave annealed (MW $5^{\circ} \mathrm{C} / \mathrm{min}$ ), and YSZ bulk disk scaffolds. (A) The formation of mineralized extracellular matrix was stained with alizarin red $S$ after 28 days of culture in BM and MM (the scale bar represents $1 \mathrm{~mm}$ ). (B) Quantitative analysis of the mineral deposits per scaffold by alizarin red S staining at day 28. Error bars represent standard deviation of the mean. ${ }^{* * *} p<0.001$. 


\section{Conclusions}

In summary, new flexible nanofibrous YSZ scaffolds that display multiscale mechanical properties were successfully fabricated by electrospinning. Their surface roughness can be tailored by employing different heat treatment method. My results demonstrate the osteogenic differentiation and mineralization of seeded human mesenchymal stromal cells was supported by the nanofibrous structure of YSZ scaffolds, in contrast to the well-known bioinert behavior of bulk YSZ. These flexible scaffolds can therefore present an appealing alternative to current brittle ceramics for bone regeneration applications.

\section{Experimental procedures}

Electrospinning. Zirconium n-propoxide (Alfa Aesar, Lancashire, U.K.) and yttrium acetate hexahydrate (Alfa Aesar, Lancashire, U.K.) were dissolved in n-propanol (Sigma Aldrich) in a 97 to $3 \%$ molar ratio. Then, polyvinyl pyrrolidone (PVP) (M.W. $1.3 \mathrm{~kg} / \mathrm{mol}$, Sigma Aldrich, St. Louis, U.S.A.) was added to the solution and left stirring overnight to ensure complete dissolution of the PVP. The solution preparation was performed in a nitrogen atmosphere. Subsequently, the solution was placed into a syringe, which was later connected to Teflon tubing with a 0.8 $\mathrm{mm}$ needle and place in the needle holder of the electrospinning setup. The flow rate was set at $1 \mathrm{~mL} / \mathrm{h}$, the air gap was $20 \mathrm{~cm}$, the voltage was $15 \mathrm{kV}$ and the temperature and the relative humidity were set to $25^{\circ} \mathrm{C}$ and $30 \%$, respectively.

Thermal annealing. The YSZ-PVP fibers were annealed to burn out the organic phase and to obtain a pure YSZ crystalline phase. The samples were annealed either in a CO (Carbolite, Hope Valley, U.K.) or a MW (Milestone MicroSynth, Shelton, U.S.A.) at different heating rates. The samples annealed in the CO were 
heated at 1 and $5{ }^{\circ} \mathrm{C} / \mathrm{min}$ to $850^{\circ} \mathrm{C}$. The samples annealed in the $\mathrm{MW}$ were either heated at $5^{\circ} \mathrm{C} / \mathrm{min}$ to $850^{\circ} \mathrm{C}$, or by RTA. RTA consisted on placing the sample directly in a pre-heated $\mathrm{MW}$ at $850{ }^{\circ} \mathrm{C}$, and after annealing the samples were cooled to room temperature without a temperature program.

Fiber morphology and diameter. The fiber morphology was studied by a Zeiss Merlin (Oberkochen, Germany) scanning electron microscope at high magnification. The fiber diameter was determined from low magnification SEM pictures. The imaging was done at $1.4 \mathrm{keV}$. Fifty measurements were performed per micrograph and per sample, respectively.

Phase identification and crystallite size. The crystallinity and phase purity of the samples were studied by $X$-ray diffraction in the $2 \theta$ range between $10^{\circ}$ and $100^{\circ}$ using a PANAlytical X'Pert (Almelo, The Netherlands), with a step size of $0.015 \circ$ and 8 s per step. The crystallite size was estimated using the Scherrer equation from the full width at half maximum of the (111) peak. The Scherrer equation is presented in equation 1.

$\tau=\frac{K \lambda}{\beta \cos \theta}$

Where $\tau$ is the crystallite size, $K$ is the dimensionless shape factor, $\lambda$ is the $X$-ray wavelength, $\theta$ the full width at half maximum of the peak and, $\vartheta$ is the Bragg's diffraction angle.

Macroscopic mechanical properties. The mechanical properties of the ceramic nanofiber mats $(n=3)$ were measured by means of microindentation. A Piuma microindenter (Optics 11, Amsterdam, The Netherlands) equipped with a tip with 
a radius of $37.5 \mu \mathrm{m}$ and a spring constant of $199 \mathrm{~N} / \mathrm{m}$ was utilized. 25 points were scanned throughout $2500 \mu \mathrm{m}^{2}$ for each sample.

Microscopic mechanical properties and surface morphology. The microscopic mechanical properties were measured by a 3-point bending test on a single nanofiber, as previously reported elsewhere [30,31]. A polycarbonate substrate with $90 \mu \mathrm{m}$ wide channels was used as a substrate to suspend the fibers. An atomic force microscope (Bruker Icon, Massachusetts, U.S.A.) equipped with a ScanAsyst-AIR (Bruker, Massachusetts, U.S.A.) tip was used to measure the force/displacement curves of the fiber. The tip had a spring constant of $0.4 \mathrm{~N} / \mathrm{m}$ and a resonance frequency of $70 \mathrm{kHz}$. The apparent Young's modulus was calculated with the Euler/Bernulli beam theory (Equation 2) using the average fiber diameter obtained from SEM.

$E^{*}=\left(\frac{F}{d}\right) \frac{L^{3}}{12 \pi r D^{4}}$

Here $E^{*}$ is the apparent Young's modulus, $F / d$ is the slope of the force displacement curve, $L$ is the gap distance and $D$ is the fiber diameter. The fiber surfaces were also scanned using the same tip in tapping mode to obtain surface morphology data. The roughness values $(\mathrm{Rq})$ were obtained by analyzing the AFM topography data using the Gwyddion software package (version 2.31).

Fatigue test. A three-point bending fatigue test was performed on the ceramic nanofiber mats $(n=3)$. This version of the 3 -point bending test was especially designed for the experiment, and consisted of (1) a plate with a well-defined gap, on which the sample was suspended on, and (2) a knife, which applied a force to deflect the sample. The gap was $2 \mathrm{~mm}$ wide and the knife was $0.5 \mathrm{~mm}$ thick. The width of the sample was kept constant at $10 \mathrm{~mm}$. The plate and knife were set on a rheometer (Anton 
Paar Physica MCR 501, Graz, Austria), which measured the force displacement curves at a speed of $50 \mu \mathrm{m} / \mathrm{s}$.

The sample's deflection was calculated assuming a trigonometric system between the sample, knife, and gap. Thus, if the displacement of the knife is known, the deflection angle may be calculated using half of the gap distance. This is exemplified in Supplementary Figure 8.

Cell Culture. Pre-selected hMSCs (donor No. 8001L) were isolated from human bone marrow by the Institute of Regenerative Medicine (Texas A\&M University, Temple, Texas) which provide standardized preparations of MSCs for research use under the auspices of an NIH/NCRR grant (P40 RR 17447-06) [54, 55]. Briefly, mononuclear cells were separated using density centrifugation and plated to obtain adherent hMSCs. The cells were harvested around a confluence of $60-80 \%$, after which they were considered as passage zero (PO). The P0 cells were further expanded and harvested to obtain passage 1 (P1). To obtain sufficient cells for scaffold-based experiments, hMSCs (P1) were expanded in T-300 flasks at a density of $3 \times 10^{5}$ cells $/ \mathrm{cm}^{2}$ and cultured in BM, consisting of $\alpha$-MEM (Gibco), 10\% fetal bovine serum (Lonza), $2 \mathrm{mM}$ L-glutamine (Gibco), $0.2 \mathrm{mM}$ ascorbic acid (Sigma), $100 \mathrm{U} / \mathrm{mL}$ penicillin and $100 \mathrm{mg} / \mathrm{mL}$ streptomycin (Gibco). The cells were harvested at approximately $80-90 \%$ confluence for further sub-cultures.

Cell seeding on scaffolds. The ceramic scaffolds were cleaned using oxygen plasma cleaning (Harrick Plasma PDC-002) for $10 \mathrm{~min}$ at high power and subsequently punched out into discs to fit inside a 24-well plate. Rubber O-rings (Eriks B.V., The Netherlands) were inserted into the well to hold the substrate on the bottom. The ceramic scaffolds were sterilized in 70\% (v/v) ethanol for $15 \mathrm{~min}$ each time for 3 times. After sterilization, the scaffolds were rinsed with PBS for 5 min each time for 3 times and immersed in $\mathrm{BM}$ at $37^{\circ} \mathrm{C}$ overnight before cell 
seeding. Ceramic scaffolds were seeded at a density of $2 \times 10^{4}$ cells $/ \mathrm{cm}^{2}$ and cultured in an incubator with $5 \% \mathrm{CO}_{2}$ humid atmosphere at $37^{\circ} \mathrm{C}$ for up to 14 days in $\mathrm{OM}$, and in both $\mathrm{BM}$ and $\mathrm{MM}$ for 28 days. BM is considered a normal growth medium, which cannot induce osteogenic differentiation nor form a mineralized matrix [39, 41]. OM consisted of BM supplemented with $10^{-8} \mathrm{M}$ dexamethasone to induce osteogenic differentiation of hMSCs and the MM consisted of OM supplemented with 6 -glycerophosphate to support the osteogenic differentiation $[39,41]$, and promote the formation of a mineralized matrix. The culture medium was refreshed every 2 days.

Live and dead assay. To determine the cell viability on scaffolds, a LIVE/DEAD assay (Life Technologies) was performed. After washing the scaffolds twice with PBS, they were incubated for 30 min with a mixture of PBS solution containing ethidium homodimer (red fluorescent)/calcein AM (green fluorescent) at room temperature following the manufacturer's protocol. Scaffolds were washed three times with PBS before imaging with a fluorescence microscope (Nikon, Eclipse E600 and Nikon DS-Filc camera).

SEM analysis. After 3 days of culture, the medium was removed, and the scaffolds were washed two times with PBS and fixed in a $10 \%$ formalin solution for $2 \mathrm{~h}$. After rinsing with PBS, the scaffolds were dehydrated with ethanol series (50\%, 70\%, 80\%, 90\%, 96\%, and 100\%). The scaffolds were further processed by critical point drying (Balzers CPD-030). The dried samples were sputtered with gold (Cressington) and imaged with a XL-30 ESEM-FEG SEM (FEI, Eindhoven, Netherlands).

Fluorescence microscopy. At day 3, samples for phalloidin staining were fixed with $4 \%(\mathrm{v} / \mathrm{v})$ paraformaldehyde (Sigma-Aldrich) in PBS, left overnight at $4{ }^{\circ} \mathrm{C}$ and subsequently washed 3 times with PBS. Afterwards, the cell membrane was 
permeabilized with $0.5 \%$ TritonX-100 solution for $10 \mathrm{~min}$ at $4{ }^{\circ} \mathrm{C}$ and washed with PBS again. A 1\% BSA in PBS solution was added for $2 \mathrm{~h}$ to block non-specific binding. After withdrawing the BSA solution, phalloidin (Alexa Fluor 488, Invitrogen; Excitation/Emission:495/518 nm) with a dilution (1:40) in 1\% BSA/PBS was applied for $25 \mathrm{~min}$ at room temperature (RT) in the dark and rinsed 3 times with PBS. For nuclei staining, DAPI (1:100) in PBS was applied for 15 min at RT in the dark. The samples were observed under a fluorescence microscope (Eclipse E600, Nikon) equipped with proper filter. The average surface area for single cells was determined by the software CellProfiler 2.1.1(Broad Institute, Cambridge, USA). The surface area in each of at least 10 cells within an image for a total of six images per sample were quantified. All these images were from the same experiment.

Cell metabolic activity. The cell metabolic activity on day 7 and day 14 was assessed using a PrestoBlue ${ }^{\mathrm{TM}}$ Cell Viability Reagent (Life technologies), according to the manufacturer's protocol. Briefly, a cultured medium was replaced with a medium containing 10\% (v/v) PrestoBlue reagent and cells were incubated for $1 \mathrm{~h}$ at $37{ }^{\circ} \mathrm{C}$. Fluorescence was measured at $590 \mathrm{~nm}$ on a Perkin Elmer Victor3 1420 multiple plate reader. Three separate experiments $(n=3)$ and two replicates each were performed.

ALP activity. The relative ALP activity was measured by chemoluminescence with a CDP-star ${ }^{\circledR}$ kit (Roche Life Science), according to the manufacturer's instruction. Briefly, cell-scaffold constructs were washed 3 times with PBS and stored at $-80^{\circ} \mathrm{C}$ for at least $24 \mathrm{~h}$. After thawing, the constructs were treated with $250 \mu \mathrm{L}$ cell lysis buffer at a pH of 7.8 (composed of $0.1 \mathrm{M} \mathrm{KH}_{2} \mathrm{OPO}_{4}, 0.1 \mathrm{M} \mathrm{K}_{2} \mathrm{HPO}_{4}$ and $0.1 \%$ Triton $\mathrm{X}-100)$ for $1 \mathrm{~h}$ at RT. Cell lysates $(50 \mu \mathrm{L})$ were transferred to Eppendorf tubes and stored at $-20^{\circ} \mathrm{C}$ for ALP assay. The remainder of the remaining cell lysates $(200 \mu \mathrm{L})$ was used for DNA assay. For ALP analysis, $10 \mu \mathrm{L}$ of cell lysates were transferred in 
duplicate to a well of a 96-well plate. Subsequently, $40 \mu \mathrm{L}$ of CDP-star were added and plates were incubated for $15 \mathrm{~min}$ at room temperature. The plates were measured on a Multilabel reader (Victor3 1420, Perkin Elmer, USA). Three separate experiments $(n=3)$ and two replicates each were performed. The results of ALP activity were presented after normalization to DNA quantity per scaffold.

Quantification of DNA amount. Cell lysates remainder, $(200 \mu \mathrm{L})$ as mentioned above, were further digested for $16 \mathrm{~h}$ at $56^{\circ} \mathrm{C}$ with $200 \mu \mathrm{L}$ Tris/EDTA buffer containing $1 \mathrm{mg} / \mathrm{mL}$ proteinase $\mathrm{K}$ (Sigma Aldrich), $185 \mu \mathrm{g} / \mathrm{mL}$ iodoacetamine (Sigma Aldrich) and $10 \mu \mathrm{g} / \mathrm{mL}$ Pepstatin A (Sigma Aldrich). The quantification of the total amount of DNA was performed using the CyQuant DNA assay (Molecular Probes, Eugene, USA) according to the manufacturer's manual. Briefly, a $40 \mu \mathrm{L}$ aliquot of lysate was pipetted in duplicate into a black 96-well plate, followed by addition of $40 \mu \mathrm{L}$ RNase (1000x) in NaCl-EDTA buffer composed of component B (20x) and incubated for $1 \mathrm{~h}$ at RT. Subsequently, $80 \mu \mathrm{L}$ of Gr-dye solution was added and incubated for $15 \mathrm{~min}$. The measurements were performed on a spectrophotometer reader (Victor3 1420, Perkin Elmer, USA) at an excitation wavelength of $480 \mathrm{~nm}$ and an emission wavelength of $520 \mathrm{~nm}$. Three separate experiments $(n=3)$ and two replicates each were performed.

Gene expression analysis. Total RNA was isolated from samples at day 7 and 14 using a combination of TRIzol with a NucleoSpinRNAll isolation kit (Bioke). Briefly, the scaffolds were washed three times with PBS and $1 \mathrm{~mL}$ of TRIzol was added for each sample. Then, $0.2 \mathrm{~mL}$ of chloroform was added and the samples were centrifuged for $15 \mathrm{~min}$ at $12,000 \mathrm{RCF}$ (relative centrifugal force). The aqueous phase containing RNA was transferred to a new Eppendorf ${ }^{\oplus}$ tube, mixed with an equal volume of $75 \%(\mathrm{v} / \mathrm{v})$ ethanol and loaded onto an RNA binding column of the isolation kit. After a series of purification procedures, the RNA was re-suspended in RNASE-free water. RNA concentration and purity were measured by using an 
ND 1000 spectrophotometer (Nanodrop Technologies, USA). Finally, cDNA was synthesized using iScript (Bio-Rad, Veenendaal, The Netherlands) according to the manufacturer's protocol. Quantitative PCR was performed using SYBR-green supermix (Bio-Rad). Four separate experiments $(n=4)$ were performed. Cycling parameters for quantitative PCR were as follows: initial denaturation for $10 \mathrm{~min}$ at $95^{\circ} \mathrm{C}$, followed by 40 cycles of $15 \mathrm{~s}$ at $95^{\circ} \mathrm{C}$ and $1 \mathrm{~min}$ at $60{ }^{\circ} \mathrm{C}$. The sequences of the analyzed primers are shown in Table 1. Cycle threshold (CT) values were normalized to the housekeeping gene $B_{2} M$, and the results of gene expression were calculated as $2^{-\Delta C T}$.

Table 1. Sequences of primers used in PCR.

\begin{tabular}{lll}
\hline Gene & Forward primer 5' to 3' & Reverse primer 5' to 3' \\
& & \\
\hline BMP-2 & ACTACCAGAAACGAGTGGGAA & GCATCTGTTCTCGGAAAACCT \\
BSP & CCCCACCTTTTGGGAAAACCA & TCCCCGTTCTCACTTTCATAGAT \\
OPN & CTCCATTGACTCGAACGACTC & CAGGTCTGCGAAACTTCTTAGAT \\
OCN & TGAGAGCCCTCACACTCCTC & CGCCTGGGTCTCTTCACTAC \\
ALP & ACAAGCACTCCCACTTCATC & TTCAGCTCGTACTGCATGTC \\
RUNX2 & TGGTTACTGTCATGGCGGGTA & TCTCAGATCGTTGAACCTTGCTA \\
COL1A1 & GAGGGCCAAGACGAAGACATC & CAGATCACGTCATCGCACAAC \\
B2M & ACAAAGTCACATGGTTCACA & GACTTGTCTTTCAGCAAGGA \\
\hline
\end{tabular}

Alizarin red S staining and quantification. After 28 days of culture, samples were washed with PBS, fixed with $10 \%$ formalin for $1 \mathrm{~h}$, and then subsequently with PBS and twice with distilled water. A freshly filtered $2 \%$ aqueous alizarin red $\mathrm{S}$ solution (pH 4.2) was applied to scaffolds for 2 min, after which the excess solution was washed off with distilled water. The quantification of orange-red coloration of alizarin red $S$ was carried out as in previous studies $[43,56]$. Briefly, constructs were transferred to a microcentrifuge tube before removing excess water. A $10 \%$ acetic acid solution was added and samples were shaken for 30 min at RT. After vortexing for $30 \mathrm{~s}$, the solution was centrifuged for $15 \mathrm{~min}$ at 20,000 RCF and the 
supernatant phase was removed to a new $1.5 \mathrm{~mL}$ microcentrifuge tube. A $10 \%$ ammonium hydroxide solution was added drop-wise to neutralize the acid. Aliquots of the supernatant phase were measured in triplicate at $405 \mathrm{~nm}$ in a 96well plate using opaque-walled, transparent-bottomed plates (Fisher Lifesciences). Three separate experiments $(n=3)$ and two replicates each were performed. Scaffolds without seeding cells were used as control.

Statistics. All data points are expressed as mean values \pm standard deviation (S.D.). Statistical analysis was carried out using GraphPad Prism 5.01 (GraphPad software, San Diego, USA) for windows. The statistical differences were determined by using two-way analysis of variance (ANOVA), followed by a Tukey's post-hoc test unless otherwise mentioned. A value of $p<0.05$ was considered statistically significant. 


\section{Supplementary Information}

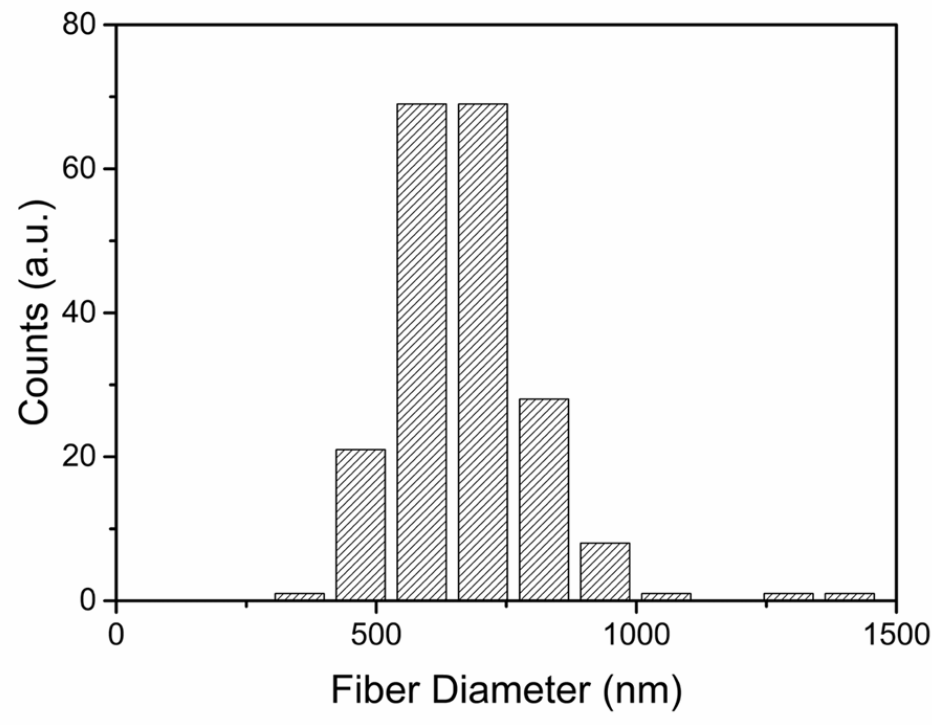

Figure S1. Fiber diameter distribution. Frequency distribution of fiber diameter as calculated from the SEM pictures. 


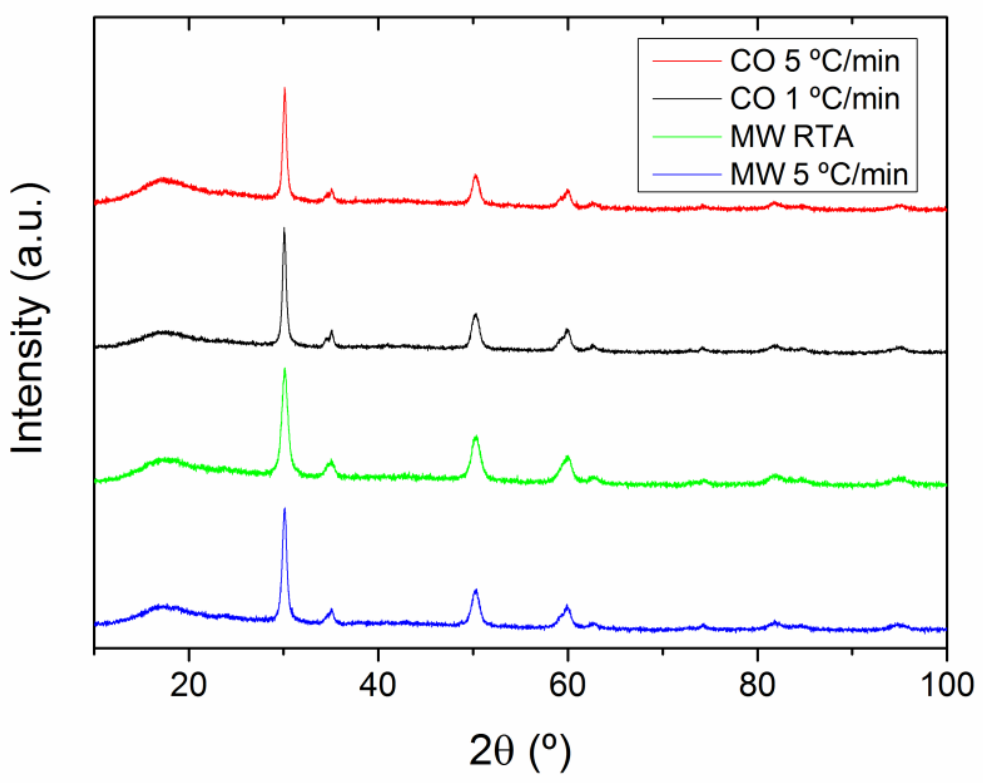

Figure S2. XRD patterns of the nanofiber mats for different annealing conditions. The XRD patterns match the crystal structure of 3\% mol yttria stabilized zirconia (ICDB 01-070-4426). CO = conventional oven; $M W=$ microwave oven; $M W R T A=$ rapid thermal annealing in microwave oven. It can be seen that the crystallinity of the samples is the same within experimental error. 

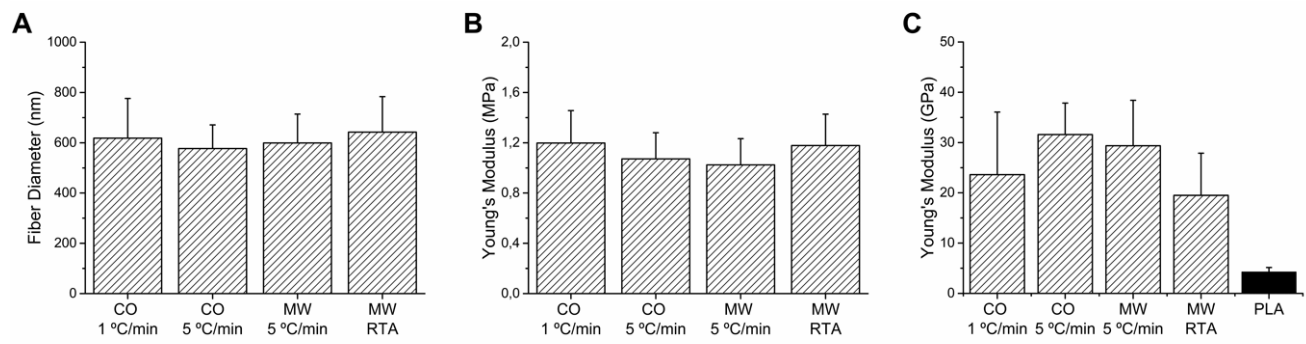

Figure S3. The average fiber diameter and Young's moduli. (A) Fiber diameter calculated from SEM images as a function of annealing procedure; (B) Young's moduli measured by microindentation; (C) Young's moduli measured by a 3 point bending test of single fibers using an AFM. Polylactic acid (PLA) nanofibers were used as reference to verify the accuracy of the measurement. Error bars represent standard deviation of the mean. The statistical significance was determined using a one way ANOVA test, where ${ }^{*} p<0.05$. CO = conventional oven; $M W=$ microwave oven; $M W R T A=$ rapid thermal annealing in microwave oven.
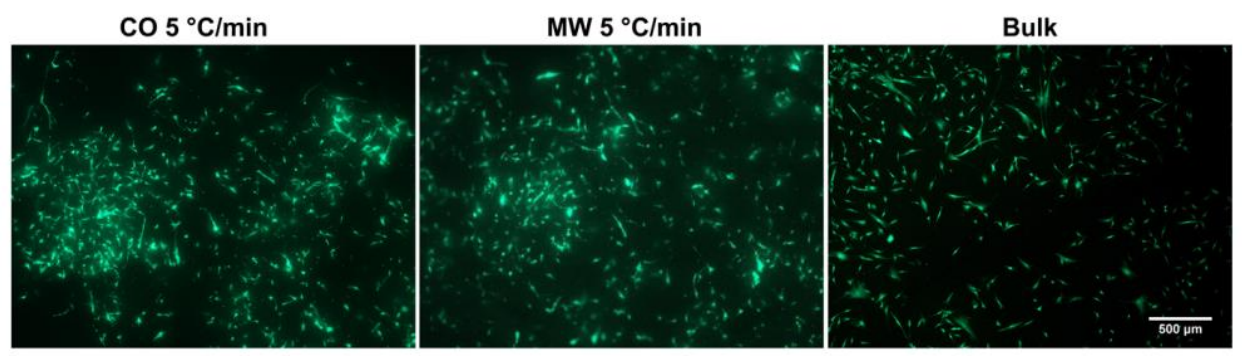

Figure S4. Live and dead assay. hMSCs viability on ceramic scaffolds at day 3 in BM. Live and dead cells are represented by green and red fluorescence, respectively. The scale bar is $500 \mu \mathrm{m}$. CO = conventional oven; $M W=$ microwave oven; $B M=$ basic medium. 
A

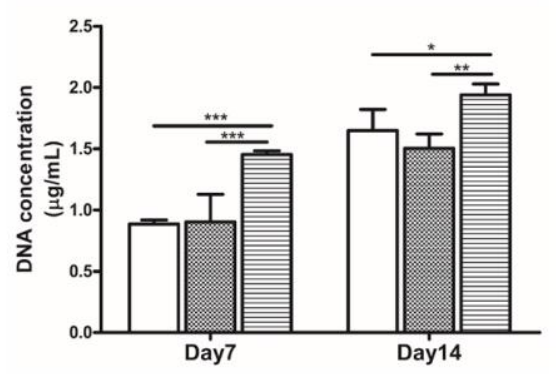

C

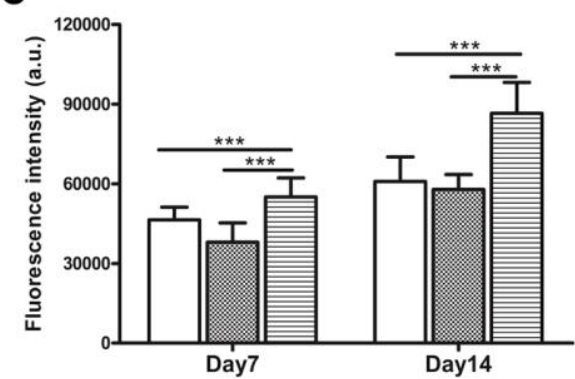

B

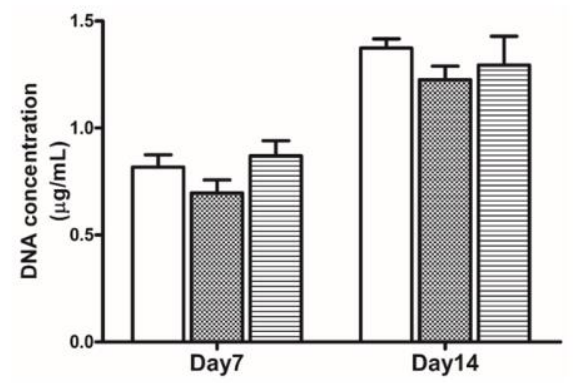

D

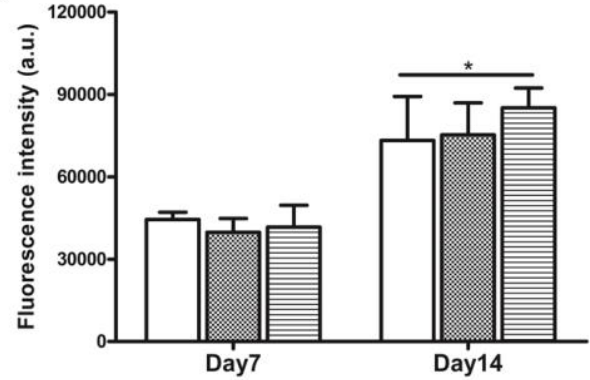

$\square \operatorname{Co~} 5^{\circ} \mathrm{C} / \mathrm{min} \quad \mathrm{MW} 5^{\circ} \mathrm{C} / \mathrm{min}$ 目 Bulk

Figure S5. DNA amounts (A-B) and metabolic activity (C-D) of hMSCs cultured on ceramic scaffolds in ( $A$ and $C) B M$ and $(B$ and $D) O M$ at day 7 and 14. Error bars represent standard deviation of the mean . ${ }^{*} p<0.05,{ }^{* * *} p<0.001$. CO = conventional oven; $M W=$ microwave oven; $B M=$ basic medium; OM=osteogenic medium. 

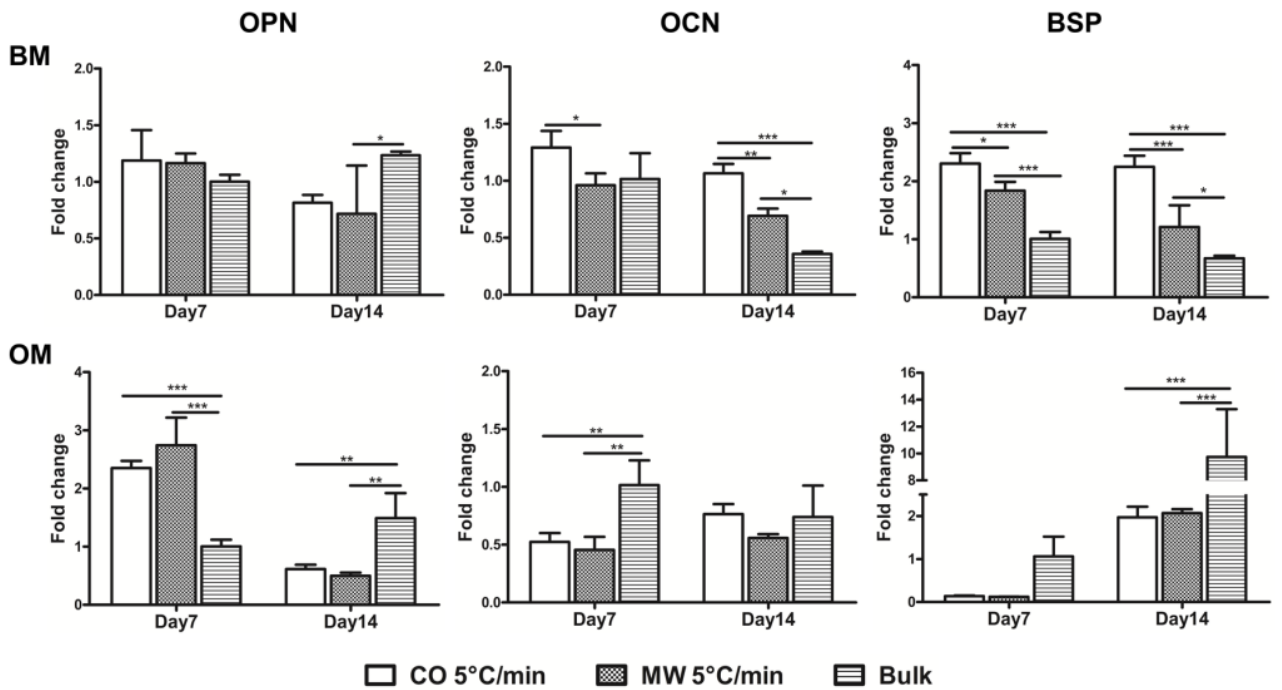

Figure S6. The expression of osteopontin (OPN), osteocalcin (OCN), and bone sialoprotein (BSP) in hMSCs cultured on scaffolds in BM and OM. The results were presented after normalizing to day 7 bulk YSZ discs in BM and OM, respectively. Error bars represent standard deviation of the mean. ${ }^{*} p<0.05,{ }^{* *} p<0.01,{ }^{* * *} p<0.001$. CO = conventional oven; $M W=$ microwave oven; $B M=$ basic medium; $O M=$ osteogenic medium. 
A
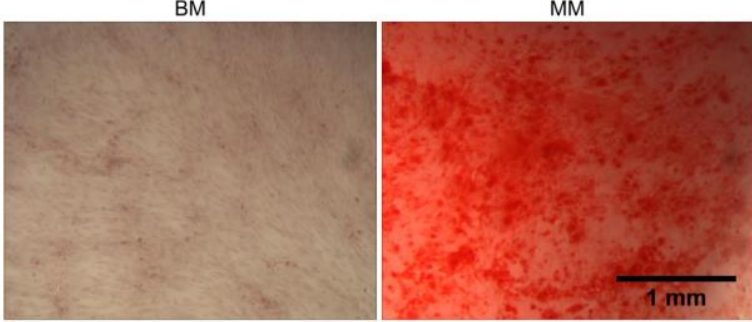

B

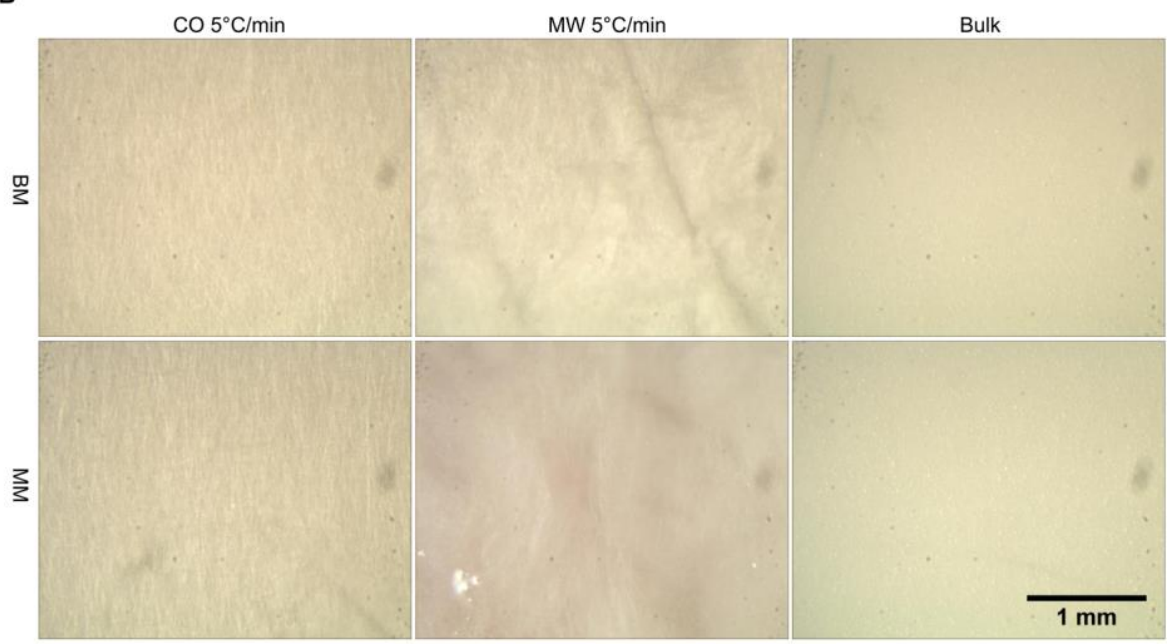

Figure S7. Calcium deposition test. (A) hMSCs cultured in a tissue culture plate as a control experiment, and stained for alizarin red $S$ after 28 days culture in $B M$ and $M M$; (B) The ceramic scaffolds without seeding $h M S C$ s incubated in BM and MM for 28 days do not stain for alizarin red $S$. $C O=$ conventional oven; $M W=$ microwave oven; $B M=$ basic medium; $M M=$ mineralization medium. 


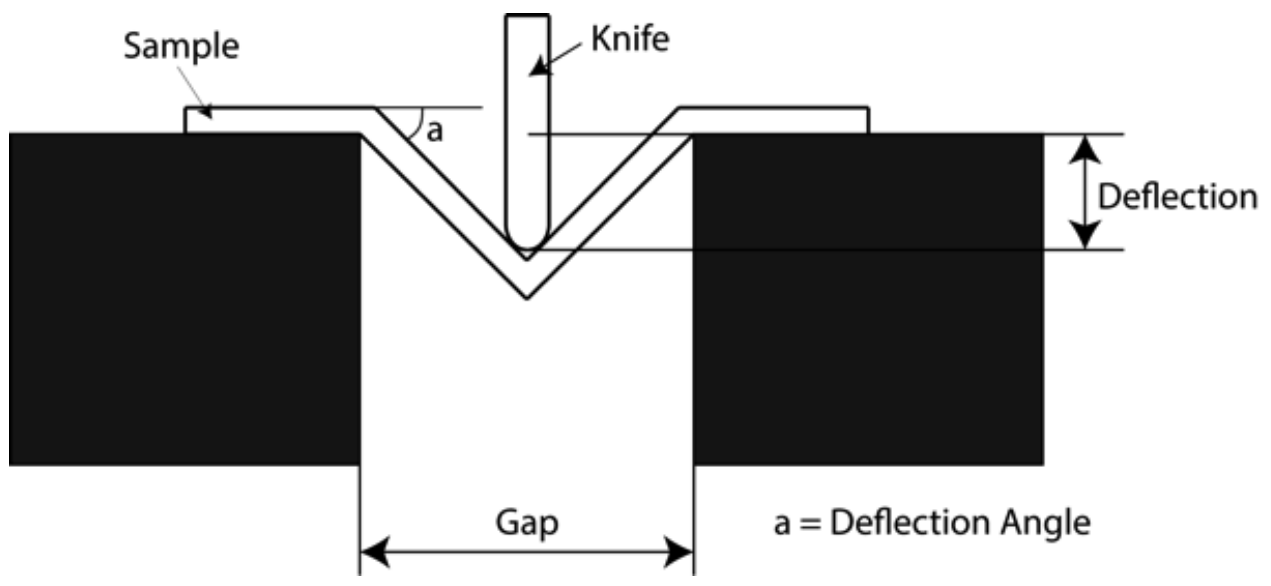

Figure S8. Schematic representation of the 3 point bending fatigue test. 


\section{REFERENCES}

1. Champion, E. Sintering of Calcium Phosphate Bioceramics. Acta Biomater. 2013, 9, 5855-5875.

2. Best, S. M.; Porter, A. E.; Thian, E. S.; Huang, J. Bioceramics: Past, Present and for the Future. J. Eur. Ceram. Soc. 2008, 28, 1319-1327.

3. Zakaria, S. M.; Sharif Zein, S. H.; Othman, M. R.; Yang, F.; Jansen, J. A. Nanophase Hydroxyapatite as a Biomaterial in Advanced Hard Tissue Engineering: A Review. Tissue Eng. Part B Rev. 2013, 19, 431-441.

4. Woodard, J. R.; Hilldore, A. J.; Lan, S. K.; Park, C. J.; Morgan, A. W.; Eurell, J. A. C.; Clark, S. G.; Wheeler, M. B.; Jamison, R. D.; Wagoner Johnson, A. J. The Mechanical Properties and Osteoconductivity of Hydroxyapatite Bone Scaffolds with Multi-Scale Porosity. Biomaterials 2007, 28, 45-54.

5. Hutmacher, D. W. Scaffolds in Tissue Engineering Bone and Cartilage. Biomaterials 2000, 21, 2529-2543.

6. Kim, H.-W.; Knowles, J. C.; Kim, H.-E. Hydroxyapatite/Poly( $\varepsilon$-caprolactone) Composite Coatings on Hydroxyapatite Porous Bone Scaffold for Drug Delivery. Biomaterials 2004, 25, 1279-1287.

7. Karageorgiou, V.; Kaplan, D. Porosity of 3D Biomaterial Scaffolds and Osteogenesis. Biomaterials 2005, 26, 5474-5491.

8. Hutmacher, D. W.; Schantz, T.; Zein, I.; Ng, K. W.; Teoh, S. H.; Tan, K. C. Mechanical Properties and Cell Cultural Response of Polycaprolactone Scaffolds Designed and Fabricated via Fused Deposition Modeling. J. Biomed. Mater. Res. 2001, 55, 203-216.

9. Piconi, C.; Maccauro, G. Zirconia As A Ceramic Biomaterial. Biomaterials $1999,20,1-25$.

10. Denry, I.; Kelly, J. R. State of The Art of Zirconia for Dental Applications. Dent. Mater. 2008, 24, 299-307.

11. Hing, K. A.; Annaz, B.; Saeed, S.; Revell, P. A.; Buckland, T. Microporosity Enhances Bioactivity of Synthetic Bone Graft Substitutes. J. Mater. Sci. Mater. Med. 2005, 16, 467-475.

12. Meza, L. R.; Das, S.; Greer, J. R. Strong, Lightweight, and Recoverable ThreeDimensional Ceramic Nanolattices. Science 2014, 345, 1322-1326. 
13. Park, S.-J.; Chase, G.; Jeong, K.-U.; Kim, H. Mechanical Properties of Titania Nanofiber Mats Fabricated by Electrospinning of Sol-Gel Precursor. J. Sol-Gel Sci. Technol. 2010, 54, 188-194.

14. Guo, M.; Ding, B.; Li, X.; Wang, X.; Yu, J.; Wang, M. Amphiphobic Nanofibrous Silica Mats with Flexible and High-Heat-Resistant Properties. J. Phys. Chem. $C$ 2009, 114, 916-921.

15. Biswas, A.; Park, H.; Sigmund, W. M. Flexible Ceramic Nanofibermat Electrospun from TiO2-SiO2 Aqueous Sol. Ceram. Int. 2012, 38, 883-886.

16. Xi, M.; Wang, X.; Zhao, Y.; Feng, Q.; Zheng, F.; Zhu, Z.; Fong, H. Mechanically Flexible Hybrid Mat Consisting of $\mathrm{TiO} 2$ and $\mathrm{SiO} 2$ Nanofibers Electrospun via Dual Spinnerets for Photo-Detector. Mater. Lett. 2014, 120, 219-223.

17. Si, Y.; Tang, X.; Ge, J.; Yang, S.; El-Newehy, M.; Al-Deyab, S. S.; Yu, J.; Ding, B. In situ Synthesis of Flexible Magnetic $\gamma$-Fe2O3@SiO2 Nanofibrous Membranes. Nanoscale 2014, 6, 2102-2105.

18. Chen, Y.; Mao, X.; Shan, H.; Yang, J.; Wang, H.; Chen, S.; Tian, F.; Yu, J.; Ding, B. Free-Standing Zirconia Nanofibrous Membranes with Robust Flexibility for Corrosive Liquid Filtration. RSC Adv. 2014, 4, 2756-2763.

19. Mao, X.; Si, Y.; Chen, Y.; Yang, L.; Zhao, F.; Ding, B.; Yu, J. Silica Nanofibrous Membranes with Robust Flexibility and Thermal Stability for High-Efficiency Fine Particulate Filtration. RSC Adv. 2012, 2, 12216-12223.

20. Wang, R.; Guo, J.; Chen, D.; Miao, Y.-E.; Pan, J.; Tjiu, W. W.; Liu, T. "Tube Brush" like $\mathrm{ZnO} / \mathrm{SiO} 2$ Hybrid to Construct a Flexible Membrane with Enhanced Photocatalytic Properties and Recycling Ability. J. Mater. Chem. 2011, 21, 19375-19380.

21. Chen, H.; Huang, J.; Yu, J.; Liu, S.; Gu, P. Electrospun Chitosan-Graft-Poly ( $\varepsilon-$ caprolactone)/Poly ( $\varepsilon$-caprolactone) Cationic Nanofibrous Mats As Potential Scaffolds for Skin Tissue Engineering. Int. J. Biol. Macromol. 2011, 48, 13-19.

22. Chen, H.; Fan, X.; Xia, J.; Chen, P.; Zhou, X.; Huang, J.; Yu, J.; Gu, P. Electrospun Chitosan-Graft-Poly ( $(\varepsilon$-caprolactone)/Poly ( $\varepsilon$-caprolactone) Nanofibrous Scaffolds for Retinal Tissue Engineering. Int. J. Nanomedicine. 2011, 6, 453-461.

23. Wade, R. J.; Bassin, E. J.; Rodell, C. B.; Burdick, J. A. Protease-Degradable Electrospun Fibrous Hydrogels. Nat. Commun. 2015, 6. 
24. Zamani, F.; Amani-Tehran, M.; Latifi, M.; Shokrgozar, M. A. The Influence of Surface Nanoroughness of Electrospun PLGA Nanofibrous Scaffold on Nerve Cell Adhesion and Proliferation. J. Mater. Sci. Mater. Med. 2013, 24, 15511560.

25. Azad, A.-M. Fabrication of Yttria-Stabilized Zirconia Nanofibers by Electrospinning. Mater. Lett. 2006, 60, 67-72.

26. Biswas, A.; Park, H.; Sigmund, W. M. Flexible Ceramic Nanofibermat Electrospun from TiO2-SiO 2 Aqueous sol. Ceram. Int. 2012, 38, 883-886.

27. Adams, J. W.; Ruh, R.; Mazdiyasni, K. Young's Modulus, Flexural Strength, and Fracture of Yttria-Stabilized Zirconia versus Temperature. J. Am. Ceram. Soc. $1997,80,903-908$.

28. Chaim, R.; Hefetz, M. Effect of Grain Size on Elastic Modulus and Hardness of Nanocrystalline $\mathrm{ZrO}_{2}-3$ wt\% $\mathrm{Y}_{2} \mathrm{O}_{3}$ Ceramic. J. Mater. Sci. 2004, 39, 3057-3061.

29. Ramesh, S.; Meenaloshini, S.; Tan, C.; Chew, W. K.; Teng, W. Effect of Manganese Oxide on The Sintered Properties and Low Temperature Degradation of Y-TZP Ceramics. Ceram. Int. 2008, 34, 1603-1608.

30. Xu, S.; Shi, Y.; Kim, S. G. Fabrication and Mechanical Property of Nano Piezoelectric Fibers. Nanotechnology 2006, 17, 4497-4501.

31. Lee, S.-H.; Tekmen, C.; Sigmund, W. M. Three-Point Bending of Electrospun TiO2 Nanofibers. Mater. Sci. Eng. A 2005, 398, 77-81.

32. Wang, J.; Binner, J.; Vaidhyanathan, B.; Joomun, N.; Kilner, J.; Dimitrakis, G.; Cross, T. E. Evidence for The Microwave Effect During Hybrid Sintering. J. Am. Ceram. Soc. 2006, 89, 1977-1984.

33. Xie, Z.; Yang, J.; Huang, X.; Huang, Y. Microwave Processing and Properties of Ceramics with Different Dielectric Loss. J. Eur. Ceram. Soc. 1999, 19, 381-387.

34. Sundaresan, S. G.; Rao, M. V.; Tian, Y.-I.; Ridgway, M. C.; Schreifels, J. A.; Kopanski, J. J. Ultrahigh-Temperature Microwave Annealing of $\mathrm{Al}^{+}-$and $\mathrm{P}^{+}$implanted 4H-SiC. J. Appl. Phys. 2007, 101.

35. Goldstein, A.; Travitzky, N.; Singurindy, A.; Kravchik, M. Direct Microwave Sintering of Yttria-Stabilized Zirconia at $2.45 \mathrm{GHz}$. J. Eur. Ceram. Soc. 1999, 19, 2067-2072.

36. Watari, S.; Hayashi, K.; Wood, J. A.; Russell, P.; Nealey, P. F.; Murphy, C. J.; Genetos, D. C. Modulation of Ssteogenic Differentiation in hMSCs Cells by 
Submicron Topographically-Patterned Ridges and Grooves. Biomaterials 2012, 33, 128-136.

37. Pot, S. A.; Liliensiek, S. J.; Myrna, K. E.; Bentley, E.; Jester, J. V.; Nealey, P. F.; Murphy, C. J. Nanoscale Topography-Induced Modulation of Fundamental Cell Behaviors of Rabbit Corneal Keratocytes, Fibroblasts, and Myofibroblasts. Invest. Ophthalmol. Vis. Sci. 2010, 51, 1373-1381.

38. McNamara, L. E.; McMurray, R. J.; Biggs, M. J.; Kantawong, F.; Oreffo, R. O.; Dalby, M. J. Nanotopographical Control of Stem Cell Differentiation. J. Tissue Eng. 2010, 1, 120623.

39. Nandakumar, A.; Yang, L.; Habibovic, P.; van Blitterswijk, C. Calcium Phosphate Coated Electrospun Fiber Matrices as Scaffolds for Bone Tissue Engineering. Langmuir 2009, 26, 7380-7387.

40. Fernandes, H.; Mentink, A.; Bank, R.; Stoop, R.; van Blitterswijk, C.; de Boer, J. Endogenous Collagen Influences Differentiation of human Multipotent Mesenchymal Stromal Cells. Tissue Eng. Part A. 2010, 16, 1693-1702.

41. Nandakumar, A.; Barradas, A.; de Boer, J.; Moroni, L.; van Blitterswijk, C.; Habibovic, P. Combining Technologies to Create Bioactive Hybrid Scaffolds for bone tissue engineering. Biomatter 2013, 3, e23705.

42. Chuah, Y. J.; Zhang, Y.; Wu, Y.; Menon, N. V.; Goh, G. H.; Lee, A. C.; Chan, V.; Zhang, Y.; Kang, Y. Combinatorial Effect of Substratum Properties on Mesenchymal Stem Cell Sheet Engineering and Subsequent Multi-Lineage Differentiation. Acta Biomater. 2015, 23,52-62.

43. Gaharwar, A. K.; Mihaila, S. M.; Swami, A.; Patel, A.; Sant, S.; Reis, R. L.; Marques, A. P.; Gomes, M. E.; Khademhosseini, A. Bioactive Silicate Nanoplatelets for Osteogenic Differentiation of human Mesenchymal Stem Cells. Adv. Mater. 2013, 25, 3329-3336.

44. Polini, A.; Pisignano, D.; Parodi, M.; Quarto, R.; Scaglione, S. Osteoinduction of Human Mesenchymal Stem Cells by Bioactive Composite Scaffolds Without Supplemental Osteogenic Growth Factors. PLoS One 2011, 6, e26211.

45. Yuan, H.; Fernandes, H.; Habibovic, P.; de Boer, J.; Barradas, A. M.; de Ruiter, A.; Walsh, W. R.; van Blitterswijk, C. A.; de Bruijn, J. D. Osteoinductive Ceramics As a Synthetic Alternative to Autologous Bone Grafting. Proc. Natl. Acad. Sci. U S A. 2010, 107, 13614-13619. 
46. Gauthier, O.; Bouler, J.-M.; Aguado, E.; Pilet, P.; Daculsi, G. Macroporous Biphasic Calcium Phosphate Ceramics:Influence of Macropore Diameter and Macroporosity Percentage on Bone Ingrowth. Biomaterials 1998, 19, 133139.

47. Scotti, C.; Tonnarelli, B.; Papadimitropoulos, A.; Scherberich, A.; Schaeren, S.; Schauerte, A.; Lopez-Rios, J.; Zeller, R.; Barbero, A.; Martin, I. Recapitulation of Endochondral Bone Formation Using Human Adult Mesenchymal Stem Cells As a Paradigm for Developmental Engineering. Proc. Natl. Acad. Sci. U S A. 2010, 107, 7251-7256.

48. Gerstenfeld, L.; Shapiro, F. Expression of Bone-Specific Genes by Hypertrophic Chondrocytes: Implications of the Complex Functions of the Hypertrophic Chondrocyte during Endochondral Bone Development. J. Cell. Biochem. 1996, 62, 1-9.

49. Kitagawa, F.; Takei, S.; Imaizumi, T.; Tabata, Y. Chondrogenic Differentiation of Immortalized Human Mesenchymal Stem Cells on Zirconia Microwell Substrata. Tissue Eng. Part C Methods 2012, 19, 438-448.

50. Curtin, C. M.; Cunniffe, G. M.; Lyons, F. G.; Bessho, K.; Dickson, G. R.; Duffy, G. P.; O'Brien, F. J. Innovative Collagen Nano-Hydroxyapatite Scaffolds Offer a Highly Efficient Non-Viral Gene Delivery Platform for Stem Cell-Mediated Bone Formation. Adv. Mater. 2012, 24, 749-754.

51. Kim, H. W.; Kim, H. E.; Knowles, J. C. Production and Potential of Bioactive Glass Nanofibers as a Next-Generation Biomaterial. Adv. Funct. Mater. 2006, $16,1529-1535$.

52. Takeuchi, K.; Saruwatari, L.; Nakamura, H. K.; Yang, J. M.; Ogawa, T. EnhancedIntrinsic Biomechanical Properties of Osteoblastic Mineralized Tissue on Roughened Titanium Surface. J. Biomed. Mater. Res. A 2005, 72, 296-305.

53. Tang, M.; Chen, W.; Liu, J.; Weir, M. D.; Cheng, L.; Xu, H. H. Human Induced Pluripotent Stem Cell-Derived Mesenchymal Stem Cell Seeding on Calcium Phosphate Scaffold for Bone Regeneration. Tissue Eng. Part A 2014, 20, 12951305.

54. DiGirolamo, C. M.; Stokes, D.; Colter, D.; Phinney, D. G.; Class, R.; Prockop, D. J. Propagation and Senescence of Human Marrow Stromal Cells in Culture: a Simple Colony-Forming Assay Identifies Samples with the Greatest Potential to Propagate and Differentiate. Br. J. Haematol. 1999, 107, 275-281. 
55. Phinney, D. G.; Kopen, G.; Righter, W.; Webster, S.; Tremain, N.; Prockop, D. J. Donor Variation in the Growth Properties and Osteogenic Potential of Human Marrow Stromal cells. J. Cell. Biochem. 1999, 75, 424-436.

56. Gregory, C. A.; Gunn, W. G.; Peister, A.; Prockop, D. J. An Alizarin Red-Based Assay of Mineralization by Adherent Cells in Culture: Comparison with Cetylpyridinium Chloride Extraction. Anal. Biochem. 2004, 329, 77-84. 


\section{Chapter 4}

\section{$\beta$-TCP Nanofiber scaffolds for bone regeneration with fine unidirectional grains}

\section{Abstract}

$\beta$-Tricalcium phosphate (TCP) nanofiber scaffolds were fabricated by electrospinning. TCP is an osteoinductive and resorbable material. Moreover, its nanofiber scaffolds are beneficial for bone tissue regeneration applications as the chemical composition resembles that of the mineral phase of natural extracellular matrix. TCP nanofibers were obtained after fine-tuning the precursor solution and thermal annealing procedure. The average fiber diameter was in the range of 100$125 \mathrm{~nm}$, which is the smallest grain size ever reported for TCP. According to previous studies, the combination of the nanofiber microstructure with small $\beta$ TCP might offer a superior biochemical activity for bone regeneration. 


\section{Introduction}

Nanofiber materials show enhanced structural and functional properties compared to bulk materials. Their aspect ratio combined with their thin fiber diameter offer a large surface area and enhanced catalytic and mechanical properties compared to other materials $[1,2]$. When a nanofibers matrix is formed, highly porous materials are obtained with a particular microstructural topography. Nanofiber materials have been successfully studied and applied in the fields of catalysis, energy, biomedicine, sensing and electronics [1-3]. In the field of biomedicine, nanofiber materials have been widely used as scaffolds for tissue regeneration.

A common technology used in regenerative medicine to fabricate nanofibers is electrospinning, which involves applying a high voltage between a hollow needle that releases a solution and a counter-electrode. The electrified solution is dragged into a fiber shape that dries during transport and is collected on the counter electrode. Electrospun ceramic nanofibers can be formed when a suitable precursor-containing solution is used.

Bioceramic materials have been extensively used to repair bone defects during the last decades [4-6]. These materials offer similar chemical and mechanical properties as bone [7-9]. Bioactive ceramics, such as calcium phosphates and bioactive glasses, have been successfully applied as they induce specific cell responses and are resorbable in some cases $[4,5,10,11]$. Among these bioactive ceramics, the $\beta$-phase of tricalcium phosphate ( $\beta$-TCP) has shown excellent performance as a scaffold for bone regeneration [10-13]. $\beta$-TCP offers osteogenic induction and is resorbable by the body itself $[10,13,14]$.

$\beta$-TCP is a much less reported phase than hydroxyapatite (HA) or calcium deficient HA ( $\mathrm{CaDHA})$. Although the mechanism of formation is not known, $\beta$-TCP results 
from decomposition of CaDHA at high temperature. Previous reports showed that TCP was formed together with secondary phases of CaDHA $[13,15]$. CaDHA started forming a secondary phase of $\beta$-TCP above $700{ }^{\circ} \mathrm{C}$. $\beta$-TCP is normally sintered above $1050{ }^{\circ} \mathrm{C}$. Such high temperatures during sintering of calcium phosphates induce grain growth, starting from small grain sizes of $250 \mathrm{~nm}$ [4].

The scaffold's microstructure plays a crucial role in its biological response. Porous $\beta$-TCP with small grain sizes has been reported to have superior properties in terms of osteogenic differentiation, inducing bone growth even in soft tissues [11, 13]. The presence of microporosity also improves osteogenesis compared to dense solid materials $[7,8]$. It is thought that microporosity provides a large surface area for cell adhesion, thereby improving the biological response [16]. Nanofiber materials fulfill such requirements and mimic the microstructure of the natural extracellular matrix, which is thought to improve cell adhesion, proliferation, and differentiation [17, 18].

Attempts to fabricate bioglass $[3,19,20]$ and $H A[21,22]$ nanofibers by electrospinning have been reported. Kim et al. reported rapid biomineralization after $6 \mathrm{~h}$ on bioglass nanofiber scaffolds when soaked in simulated body fluid, as well as excellent osteoblast response [3]. However, to the best of my knowledge no attempt to fabricate $\beta$-TCP nanofibers has been reported.

We demonstrate in this study the successful fabrication of $\beta$-TCP nanofibers by electrospinning. I assessed the critical steps to successfully prepare $\beta$-TCP and characterized the resulting scaffolds. 


\section{Materials and Methods}

TCP was synthetized using an acid-base method between calcium nitrate tetrahydrate (Sigma Aldrich) and phosphoric acid (Sigma Aldrich). A 1 M calcium nitrate tetrahydrate solution was prepared in 1:1 vol/vol ethanol:water. Separately, a $0.67 \mathrm{M}$ phosphoric acid solution was prepared in 4:1 vol/vol ethanol:water. Then, the solution was stabilized via two different strategies, namely by adding a third solution consisting of either (i) acetic acid (HAc) (Sigma Aldrich) acting as chelating agent, or (ii) 1:1 vol/vol ethanol:water to limit the concentration of free ions. The three solutions containing the calcium precursor, the phosphorus precursor and the stabilizer were mixed in a volume ratio 2:2:1.

The electrospinning process was performed in a homemade setup at $25{ }^{\circ} \mathrm{C}$ and a relative humidity of $30 \%$. The spinneret had an inner diameter of $0.8 \mathrm{~mm}$ and the distance from spinneret to collector plate was $17 \mathrm{~cm}$.

When acetic acid was used as stabilizer, the flow rate was set to $0.6 \mathrm{~mL} / \mathrm{h}$ and the voltage to $15 \mathrm{kV}$. When the solution without acetic acid was used, the flow rate and the voltage were reduced to $0.25 \mathrm{~mL} / \mathrm{h}$ and $12 \mathrm{kV}$, respectively.

The samples were annealed either in a convection oven (Eurotherm) or a microwave oven (Milestone MicroSynth). The annealing temperatures ranged from 950 to $1300{ }^{\circ} \mathrm{C}$, the dwell time was set to either 0 or $1.5 \mathrm{~h}$, and the heating and cooling rates were set to 5,10 or $20^{\circ} \mathrm{C} / \mathrm{min}$.

A Zeiss Merlin Scanning electron microscope (SEM) was used to investigate the microstructure of the prepared material. A PANalytical X'Pert X-Ray Diffraction (XRD) machine was used for phase analysis. The XRD data were analyzed with High Score Plus ${ }^{\circ}$. 


\section{Results and discussion}

Solutions for electrospinning are normally weak electrolytes (i.e. ion free solutions) with low conductivity, of the order of few tens of $\mu \mathrm{S} / \mathrm{cm}$ [23]. Addition of metal oxide precursor salts increases conductivity, which complicates the electrospinning process. The use of complexing agents to lower the effective ionic strength facilitates the spinning of highly conductive solutions.

In the first strategy, I used HAc as solvent and chelating agent. The flow rate was set to $0.6 \mathrm{~mL} / \mathrm{h}$ and the voltage to $15 \mathrm{kV}$. The spinning process improved considerably when HAc was used, compared to the solution without any chelating agent. However, a CaDHP phase was obtained instead of $\beta$-TCP, as shown in Figure $1 \mathrm{~A}$. It is known that the formation of $\beta$-TCP is complicated by the phase transition from CaDHA [15]. CaDHA is a stable phase, which transforms into $\alpha$-TCP when annealed at high temperatures, e.g. $1200{ }^{\circ} \mathrm{C}$ [15]. To obtain $\beta$-TCP, the CaDHA phase should degrade at $950{ }^{\circ} \mathrm{C}$. Therefore, the stabilization of ions by a chelation agent like HAc hinders $\beta$-TCP formation.

In the second strategy, in the absence of HAc, $\beta$-TCP was successfully formed (Figure 1A). However, the spinning process did not result in a uniform deposition process, so the voltage and the flow were reduced to $12 \mathrm{kV}$ and $0.25 \mathrm{~mL} / \mathrm{h}$ to keep the jet stable. The required reduction of voltage and flow rate to stabilize the jet was attributed to the fact that the non-stabilized ionic charges dissociated at lower voltages, which increased the electrostatic repulsion between segments of the fiber and resulted in unstable whipping of the jet [24]. The lower flow rate resulted in a lower amount of charged spices between the spinneret and the collector. This helped to stabilize the jet in the electric field.

It has been reported that the sintering of bulk $\beta$-TCP starts above $750{ }^{\circ} \mathrm{C}$, the maximum sintering rate is at $950-1050{ }^{\circ} \mathrm{C}$, and the highest densities are 
achieved above $1100{ }^{\circ} \mathrm{C}$ [4]. TCP is therefore usually sintered at temperatures higher than $1050^{\circ} \mathrm{C}$, without application of external pressure. However, when the electrospun green fibers were annealed at $1050{ }^{\circ} \mathrm{C}$, the resulting ceramic fibers were not continuous (Figure 1B) due to sintering (surface area reduction) effects. The phenomenon was even more pronounced for the fibers annealed at $1300{ }^{\circ} \mathrm{C}$. The fibers lost their elongated shape and became more rounded (figure $1 \mathrm{C}$ ). The microstructure resembled that of porous CaDHA [4, 25]. High surface area nanofibers could be formed only at temperatures lower than $1000^{\circ} \mathrm{C}$.

Short thermal treatments at high temperatures are expected to induce crystallization while avoiding surface area reduction and/or grain growth. I heated the nanofibers to $950{ }^{\circ} \mathrm{C}$ and then cooled them down immediately with heating/cooling rates of 5,10 or $20{ }^{\circ} \mathrm{C} / \mathrm{min}$. Both microwave and convection ovens were used. The convection oven was not able to heat or cool faster than 10 ${ }^{\circ} \mathrm{C} / \mathrm{min}$. The results are presented in Figure 2 . It is noted that samples annealed with a dwell time of $0 \mathrm{~h}$ did not show formation of $\beta$-TCP but only CaDHA. However, the purpose of these experiments was merely to investigate the influence of heating/cooling rate on fiber morphology. Fibers annealed with heating/cooling rates of $5{ }^{\circ} \mathrm{C} / \mathrm{min}$ were discontinuous, whereas the fibers annealed with heating/cooling rates of $20^{\circ} \mathrm{C} / \mathrm{min}$ were continuous but porous. Annealing with intermediate heating/cooling rates of $10{ }^{\circ} \mathrm{C} / \mathrm{min}$ were continuous and dense. The annealing method, i.e. heating via microwave radiation or convection, did not have a significant influence on the fiber morphology. It can be concluded that higher heating/cooling rates help to maintain the nanofiber structure. In the initial stage of sintering, interparticle necks are formed. However, the initial sintering stage is short compared to the other two stages in which densification and grain growth occur, respectively [4]. High heating and cooling rates guarantee a short exposure to high temperature, enough to densify the 
structure but to avoid grain growth and maintain the nanofibrous microstructure. The average fiber diameter was in the range of $100-125 \mathrm{~nm}$ for all the samples. The heating mechanism and heating/cooling rate did not have a significant effect on the final fiber diameter (Figure 2F).
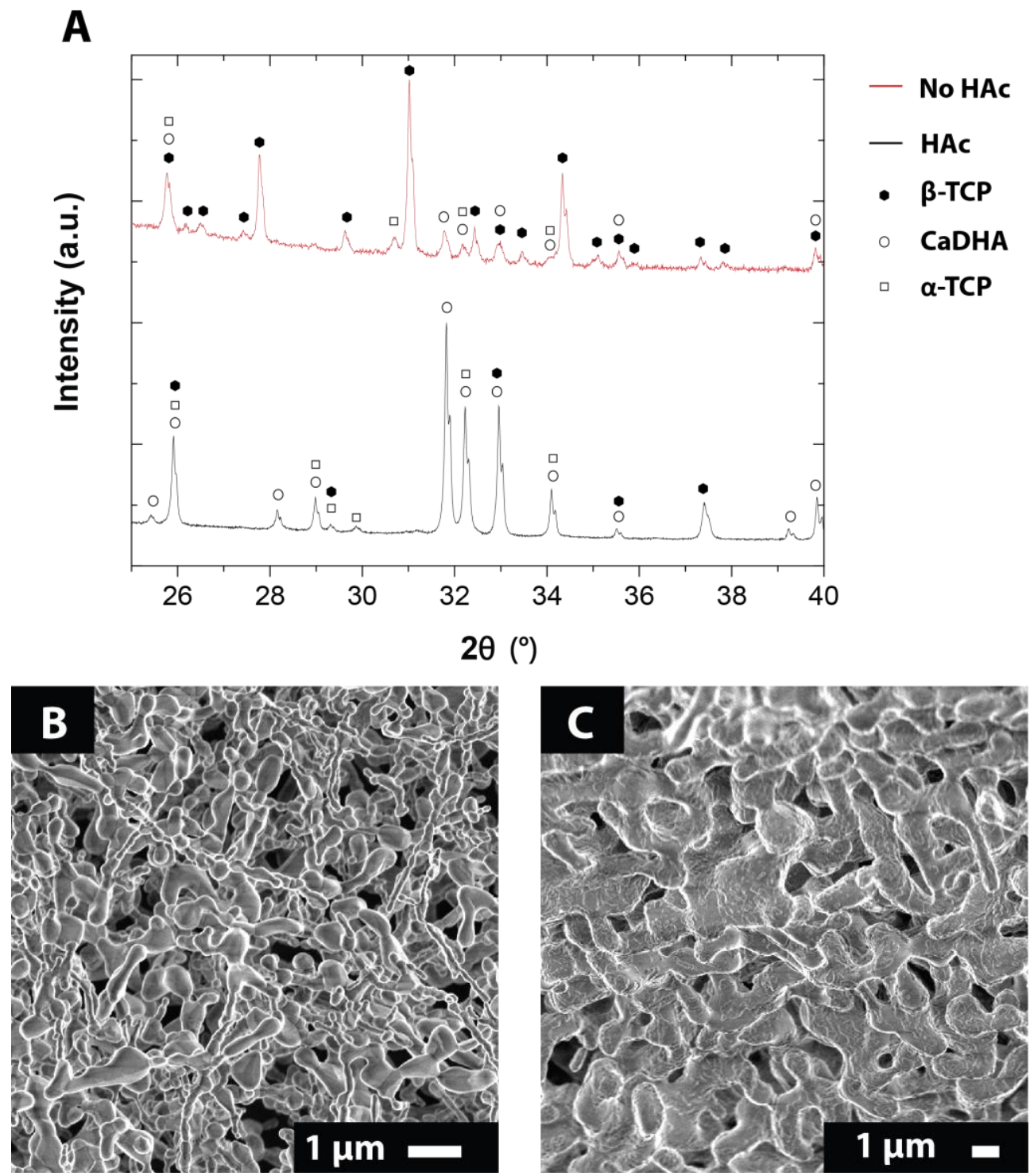

Figure 1. (A) XRD patterns of samples spun with and without acetic acid as stabilizer. The samples were annealed at $1050^{\circ} \mathrm{C}$. (B) SEM image of a sample spun without using acetic acid and annealed at $1050^{\circ} \mathrm{C}$. (C) SEM image of a sample spun without acetic acid and annealed at $1300{ }^{\circ} \mathrm{C}$. The scale bars are $1 \mu \mathrm{m}$. 

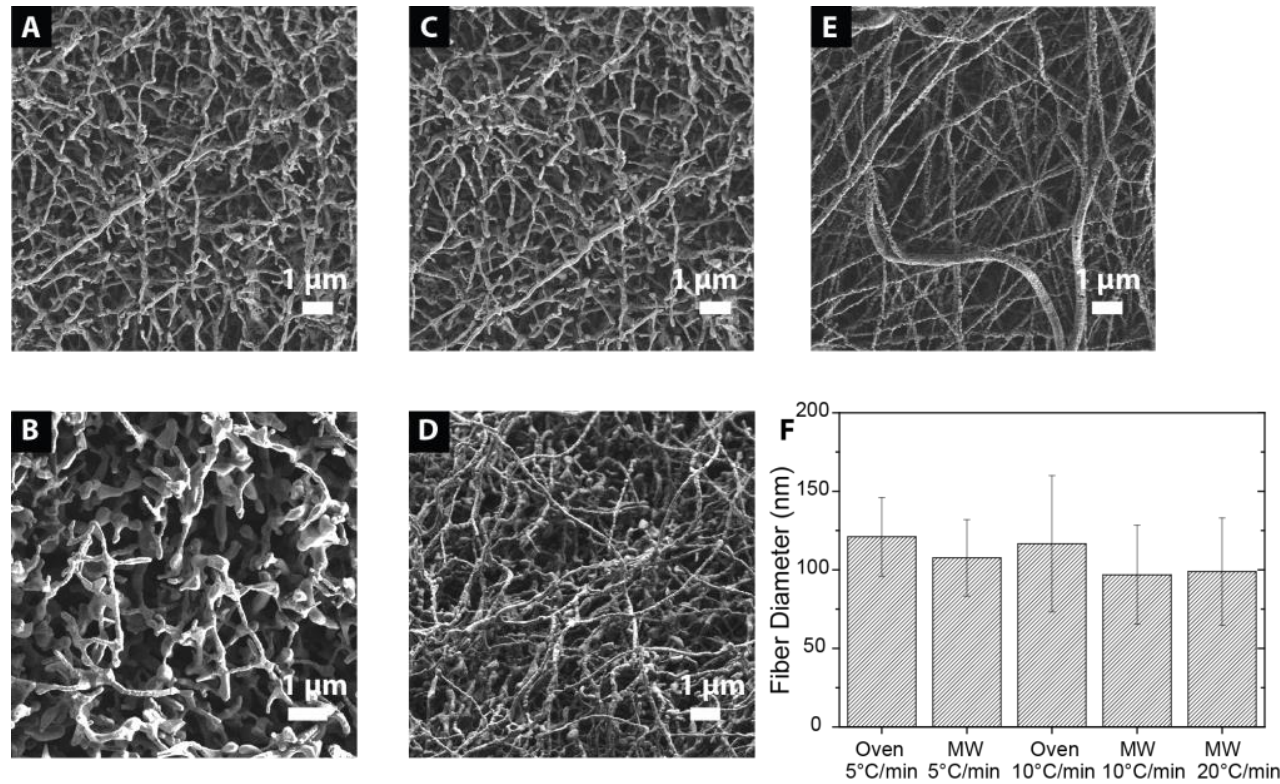

Figure 2. SEM images of calcium phosphate nanofibers annealed up to $950{ }^{\circ} \mathrm{C}$ at different

heating/cooling rates in a microwave (MW) or a convection oven (oven): (A) MW $5^{\circ} \mathrm{C} / \mathrm{min}$, (B) Oven $5{ }^{\circ} \mathrm{C} / \mathrm{min},(\mathrm{C}) \mathrm{MW} 10^{\circ} \mathrm{C} / \mathrm{min}$, (D) Oven $10^{\circ} \mathrm{C} / \mathrm{min}$, (E) MW $20^{\circ} \mathrm{C} / \mathrm{min}$. (F) Average fiber diameter of the samples shown in Figure 2 A-E.

The influence of dwell time on $\beta$-TCP phase formation was studied by varying the dwell time from 0 to $1.5 \mathrm{~h}$, while keeping the heating/cooling rates at their maximum values for both the convection oven $\left(10^{\circ} \mathrm{C} / \mathrm{min}\right)$ and the microwave oven $\left(20^{\circ} \mathrm{C} / \mathrm{min}\right)$.

$\beta$-TCP was obtained as the main phase when the samples were annealed for $1.5 \mathrm{~h}$ in either oven. The results are presented in Figure 3A. However, HA and $\alpha-$ TCP secondary phases were also formed, especially in the sample that had been annealed in a convection oven. The better phase purity of the microwave-treated material can be attributed to the effect of microwave radiation heating, also known as the microwave effect, that allows better crystallization [26-29]. Simular secondary phases have also been reported in previous studies on TCP formation $[13,15]$. It can thus be concluded that the $\beta$-TCP phase with similar purity as previously reported elsewhere can be shaped into nanofibers. 
The samples that were thermally annealed in the microwave oven (Figure 3B) kept its nanofiber morphology better than the ones annealed in a convection oven (Figure $3 \mathrm{C}$ ), which is attributed to the faster heating/cooling rate of the microwave oven as discussed above. The fiber diameter of the samples annealed for $1.5 \mathrm{~h}$ in a microwave and a convection oven had fiber diameters of $120 \pm 20$ and $120 \pm 30 \mathrm{~nm}$, respectively. Since the fiber diameter did not change significantly with annealing time, the degree of densification was the same in both cases.

The grains had a size of approximately $100 \mathrm{~nm}$ as presented in Figure 4, which is close to the fiber diameter. This value is much smaller than normally reported grain sizes for $\beta$-TCP, which are of the order of micrometers, and about 2.5 smaller than the smallest reported $\beta$-TCP grain sizes known to me [4].

The grains were clearly anisotropic, directionally oriented along the long axis of the fibers (Figure 4). This is probably caused by the lack of mass transport towards the grain from the surrounding matrix during sintering, which would not allow grain growth in other directions but the fiber direction itself [4], in combination with the relatively short sintering time and low sintering temperature. 


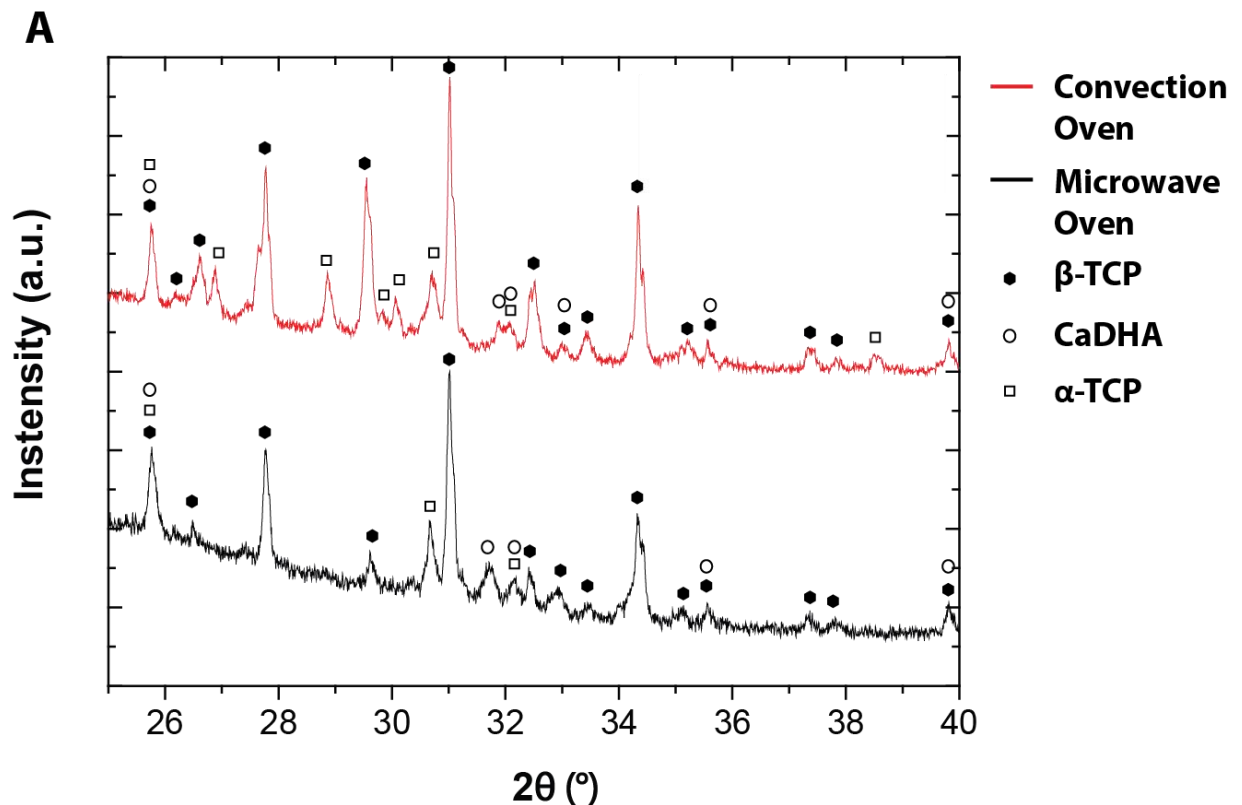

\section{Microwave Oven}

Convection Oven
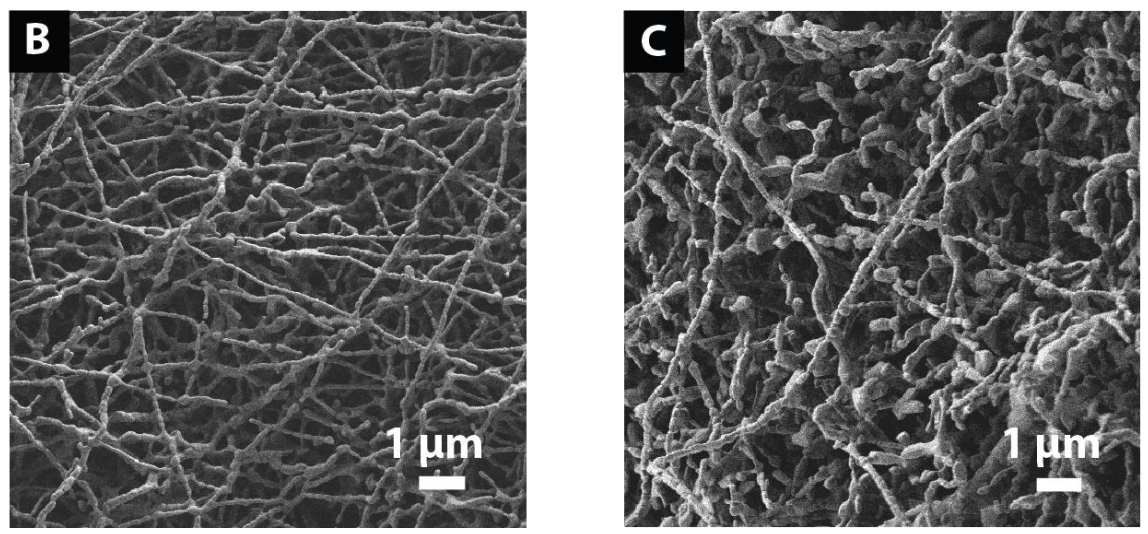

Figure 3. (A) XRD patterns of the samples shown in (B) and (C), respectively. (B) SEM image of a sample annealed in a microwave oven at $950{ }^{\circ} \mathrm{C}$ for $1.5 \mathrm{~h}$ and a heating/cooling rate of $20{ }^{\circ} \mathrm{C} / \mathrm{min}$. The scale bar is $1 \mu \mathrm{m}$. (C) SEM image of a sample annealed in a convection oven at $950{ }^{\circ} \mathrm{C}$ for $1.5 \mathrm{~h}$ and a heating/cooling rate of $10{ }^{\circ} \mathrm{C} / \mathrm{min}$. The scale bar is $1 \mu \mathrm{m}$. 


\section{Microwave Oven}

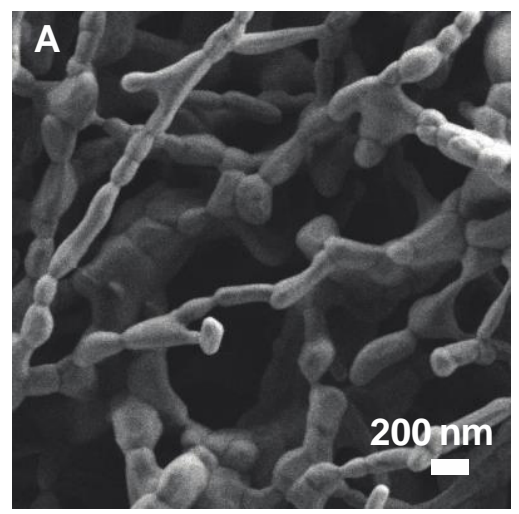

Convection Oven

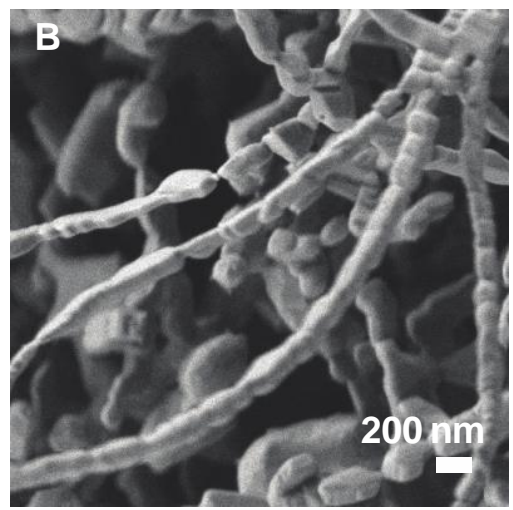

Figure 4. Formation of elongated grains. (A) Sample annealed in a microwave oven for $1.5 h$ at a heating/cooling rate of $20{ }^{\circ} \mathrm{C} / \mathrm{min}$. The scale bar is $200 \mathrm{~nm}$. (B) Sample annealed in convection oven for $1.5 \mathrm{~h}$ at a heating/cooling rate of $10^{\circ} \mathrm{C} / \mathrm{min}$. The scale bar is $200 \mathrm{~nm}$. 


\section{Conclusions}

Nearly phase-pure $\beta$-TCP nanofiber mats were successfully fabricated. To the best of my knowledge, this is the first time that $\beta$-TCP nanofibers have been reported. The grain sizes were of the order of $100 \mathrm{~nm}$, about 2.5 times smaller than the smallest $\beta$-TCP grain sizes reported till date. Rapid heating/cooling and short sintering times help to maintain small grain sizes. A sintering time of $1.5 \mathrm{~h}$ was required to obtain transform the fiber into dense $\beta$-TCP fibers without grain growth phenomena. The nanofiber morphology promoted unidirectional grain growth during the sintering stage due to lack of surrounding grains in the other two principal directions.

$\beta$-TCP nanofiber mats may offer an excellent platform for bone regeneration studies. Both microstructure and composition are optimized, which can be tested in future studies for osteogenic differentiation and rapid bone recovery. 


\section{References}

1. Wu, H., W. Pan, D. Lin, and H. Li, Electrospinning of ceramic nanofibers: Fabrication, assembly and applications. Journal of Advanced Ceramics, 2012. 1(1): p. 2-23.

2. Li, D., J.T. McCann, Y. Xia, and M. Marquez, Electrospinning: A Simple and Versatile Technique for Producing Ceramic Nanofibers and Nanotubes. Journal of the American Ceramic Society, 2006. 89(6): p. 1861-1869.

3. Kim, H.W., H.E. Kim, and J.C. Knowles, Production and Potential of Bioactive Glass Nanofibers as a Next-Generation Biomaterial. Advanced Functional Materials, 2006. 16(12): p. 1529-1535.

4. Champion, E., Sintering of calcium phosphate bioceramics. Acta Biomaterialia, 2013. 9(4): p. 5855-5875.

5. Best, S.M., A.E. Porter, E.S. Thian, and J. Huang, Bioceramics: Past, present and for the future. Journal of the European Ceramic Society, 2008. 28(7): p. 1319-1327.

6. Zakaria, S.M., S.H. Sharif Zein, M.R. Othman, F. Yang, and J.A. Jansen, Nanophase Hydroxyapatite as a biomaterial in advanced hard tissue engineering: A review. Tissue Engineering - Part B: Reviews, 2013. 19(5): p. 431-441.

7. Woodard, J.R., A.J. Hilldore, S.K. Lan, C.J. Park, A.W. Morgan, J.A.C. Eurell, S.G. Clark, M.B. Wheeler, R.D. Jamison, and A.J. Wagoner Johnson, The mechanical properties and osteoconductivity of hydroxyapatite bone scaffolds with multi-scale porosity. Biomaterials, 2007. 28(1): p. 45-54.

8. Karageorgiou, V. and D. Kaplan, Porosity of 3D biomaterial scaffolds and osteogenesis. Biomaterials, 2005. 26(27): p. 5474-5491.

9. Hutmacher, D.W., Scaffolds in tissue engineering bone and cartilage. Biomaterials, 2000. 21(24): p. 2529-2543.

10. Yuan, H., J.D. De Bruijn, Y. Li, J. Feng, Z. Yang, K. De Groot, and X. Zhang, Bone formation induced by calcium phosphate ceramics in soft tissue of dogs: a comparative study between porous $\alpha$-TCP and B-TCP. Journal of Materials Science: Materials in Medicine, 2001. 12(1): p. 7-13.

11. Yuan, H., K. Kurashina, J.D. de Bruijn, Y. Li, K. de Groot, and X. Zhang, A preliminary study on osteoinduction of two kinds of calcium phosphate ceramics. Biomaterials, 1999. 20(19): p. 1799-1806. 
12. Yuan, H., Z. Yang, J.D. de Bruijn, K. de Groot, and X. Zhang, Materialdependent bone induction by calcium phosphate ceramics: a 2.5-year study in dog. Biomaterials, 2001. 22(19): p. 2617-2623.

13. Yuan, H., H. Fernandes, P. Habibovic, J. de Boer, A.M.C. Barradas, A. de Ruiter, W.R. Walsh, C.A. van Blitterswijk, and J.D. de Bruijn, Osteoinductive ceramics as a synthetic alternative to autologous bone grafting. Proceedings of the National Academy of Sciences of the United States of America, 2010. 107(31): p. 13614-13619.

14. Lu, J., M. Descamps, J. Dejou, G. Koubi, P. Hardouin, J. Lemaitre, and J.-P. Proust, The biodegradation mechanism of calcium phosphate biomaterials in bone. Journal of Biomedical Materials Research, 2002. 63(4): p. 408412.

15. Cicek, G., E. Aksoy, C. Durucan, and N. Hasirci, Alpha-tricalcium phosphate $(\alpha-T C P)$ : solid state synthesis from different calcium precursors and the hydraulic reactivity. Journal of Materials Science: Materials in Medicine, 2011. 22(4): p. 809-817.

16. Kuboki, Y., H. Takita, D. Kobayashi, E. Tsuruga, M. Inoue, M. Murata, N. Nagai, Y. Dohi, and H. Ohgushi, BMP-induced osteogenesis on the surface of hydroxyapatite with geometrically feasible and nonfeasible structures: Topology of osteogenesis. Journal of Biomedical Materials Research, 1998. 39(2): p. 190-199.

17. Ma, Z., M. Kotaki, R. Inai, and S. Ramakrishna, Potential of nanofiber matrix as tissue-engineering scaffolds. Tissue Engineering, 2005. 11(1-2): p. 101-109.

18. Vasita, R. and D.S. Katti, Nanofibers and their applications in tissue engineering. International Journal of Nanomedicine, 2006. 1(1): p. 15-30.

19. Lu, H., T. Zhang, X.P. Wang, and Q.F. Fang, Electrospun submicron bioactive glass fibers for bone tissue scaffold. Journal of Materials Science: Materials in Medicine, 2009. 20(3): p. 793-798.

20. Xia, W., D. Zhang, and J. Chang, Fabrication and in vitro biomineralization of bioactive glass (BG) nanofibres. Nanotechnology, 2007. 18(13): 135601.

21. Dai, X. and S. Shivkumar, Electrospinning of PVA-Calcium Phosphate Sol Precursors for the Production of Fibrous Hydroxyapatite. Journal of the American Ceramic Society, 2007. 90(5): p. 1412-1419.

22. Mouthuy, P.-A., A. Crossley, and H. Ye, Fabrication of calcium phosphate fibres through electrospinning and sintering of hydroxyapatite nanoparticles. Materials Letters, 2013. 106(0): p. 145-150. 
23. Collins, G., J. Federici, Y. Imura, and L.H. Catalani, Charge generation, charge transport, and residual charge in the electrospinning of polymers: A review of issues and complications. Journal of Applied Physics, 2012. 111(4): 044701.

24. Collins, G., J. Federici, Y. Imura, and L.H. Catalani, Charge generation, charge transport, and residual charge in the electrospinning of polymers: A review of issues and complications. Journal of Applied Physics, 2012. 111(4): p. 044701.

25. Habibovic, P., H. Yuan, C.M. van der Valk, G. Meijer, C.A. van Blitterswijk, and $\mathrm{K}$. de Groot, 3D microenvironment as essential element for osteoinduction by biomaterials. Biomaterials, 2005. 26(17): p. 3565-3575.

26. Wang, J., J. Binner, B. Vaidhyanathan, N. Joomun, J. Kilner, G. Dimitrakis, and T.E. Cross, Evidence for the microwave effect during hybrid sintering. Journal of the American Ceramic Society, 2006. 89(6): p. 1977-1984.

27. Wroe, R. and A.T. Rowley, Evidence for a non-thermal microwave effect in the sintering of partially stabilized zirconia. Journal of Materials Science, 1996. 31(8): p. 2019-2026.

28. Xie, Z., J. Yang, X. Huang, and Y. Huang, Microwave processing and properties of ceramics with different dielectric loss. Journal of the European Ceramic Society, 1999. 19(3): p. 381-387.

29. Bhaskar, A., T.H. Chang, H.Y. Chang, and S.Y. Cheng, Low-temperature crystallization of sol-gel-derived lead zirconate titanate thin films using 2.45 GHz microwaves. Thin Solid Films, 2007. 515(5): p. 2891-2896. 


\section{Chapter 5}

\section{Low-cost, large-area, \\ facile and rapid \\ fabrication of aligned \\ ZnO nanowire device \\ arrays}

\section{Abstract}

Well aligned nanowires of $\mathrm{ZnO}$ have been made with an electrospinning technique using zinc acetate precursor solutions. Employment of two connected parallel collector plates with a separating gap of $4 \mathrm{~cm}$ resulted in a very high degree of nanowire alignment. By adjusting the process parameters, the deposition density of the wires could be controlled. Field effect transistors were prepared by depositing wires between two gold electrodes on top of a heavily doped $\mathrm{Si}$ substrate covered with a $300 \mathrm{~nm}$ oxide layer. These devices showed good FET characteristics and photo-sensitivity under UV-illumination. The method provides a fast and scalable fabrication route for functional nanowire arrays with a high degree of alignment and control over nanowire spacing. 


\section{Introduction}

Nanowires of inorganic materials have attracted huge interest over the last decade because of their wide range of applications in the areas of electronics, optoelectronics and sensing. Among other synthesis methods, electrospinning has evolved as an excellent tool for large scale, low cost and relatively fast manufacturing of nanofibers and nanowires over the past decade. Nanowires of a wide range of functional materials, both polymeric and ceramic, have been fabricated by electrospinning and have found application in human tissue engineering [1], energy storage devices [2] and sensing [3]. Usually these nanowires are formed as mats of non-oriented ultra-long fibers/wires. For the aforementioned applications, alignment is not relevant. However, for the manufacture of low cost electronic, optoelectronic and sensing device arrays, unidirectional alignment of nanowires is essential. Aligned wires allow easy connection of external electrodes at both ends of all nanowires, facilitating the ease and reproducibility of the fabrication process considerably. The first reports of ceramic nanofibers prepared by electrospinning date back to 2002 [4]. One year later, Li et al. reported the fabrication of ceramic arrays of aligned nanowires by electrospinning [5]. Since then, there have been reports of devices based on arrays of electrospun nanowires, such as field effect transistors [6], mechanical energy harvesters [7], supercapacitors [8], photosensors [9], etc. However, these devices were limited to surface areas of a few square micrometers $\left(\sim 10 \mu \mathrm{m}^{2}\right)$. Although the mechanism of fiber alignment in an electrospinning process has been modeled [10], only experimental studies without control over nanowire spacing have been reported [11].

The electrospinning process has certain advantages over common nanowire fabrication methods including vapor-liquid solid (VLS) growth, hydrothermal synthesis, and template assisted electrodeposition, since the latter methods 
cannot produce nanowires with planar alignment on a device substrate. The VLS growth and hydrothermal synthesis methods yield nanowires grown vertically on a substrate. In the case of template assisted electrodeposition, nanowires are electrodeposited in an anodic alumina or polymeric template, followed by template removal. In order to make planar electronic or sensing devices, one then has to disperse the nanowires in a solution and drop cast on a device substrate to obtain horizontally oriented nanowires. Such device fabrication approaches are time consuming and only prototype scale devices can be obtained.

In this contribution I demonstrate that electrospinning can be used as a fast fabrication tool to form large area device arrays of highly aligned ceramic nanowires with a high degree of control over density, shape, and spacing. The process of fabrication of the nanowire devices is shown in Figure 1. Briefly, electrospinning consists of a spinneret connected to a collector counter electrode via a high voltage power supply. When a viscous and moderately conductive precursor solution is pumped into the spinneret, it becomes electrified under the influence of the high voltage and stretches into a nanofiber, due to both the electrostatic attraction by the counter electrode and the mutually repulsive electrostatic interactions at the nanofiber surface. The nanofiber dries on its way to the counter electrode where it is collected [12]. A thermal treatment is subsequently applied on these preceramic hybrid wires to convert them into the final metal oxide phase. The method can be used for fast synthesis of aligned oxide nanowire arrays over wafer scale surface areas, and can thus have many potential applications in electronics, optoelectronics and sensing. The technique can be employed for a wide range of functional oxides. Here, I chose $\mathrm{ZnO}$ as a model system to demonstrate the ability of the technique to fabricate large area aligned arrays in a very short period of time, and illustrate their applicability by making field effect transistors and photo-detector arrays. Aligned electrospun 
oxide nanowire arrays for photosensors have been demonstrated before $[13,14]$, but the main advance of my method is that it provides a very fast route with which wafer scale nanowire arrays $\left(4 \times 4 \mathrm{~cm}^{2}\right.$, see supporting information figure S1) with a high degree of alignment and control over nanowire spacing were obtained, and that is further scalable to industrial scale manufacturing of devices.

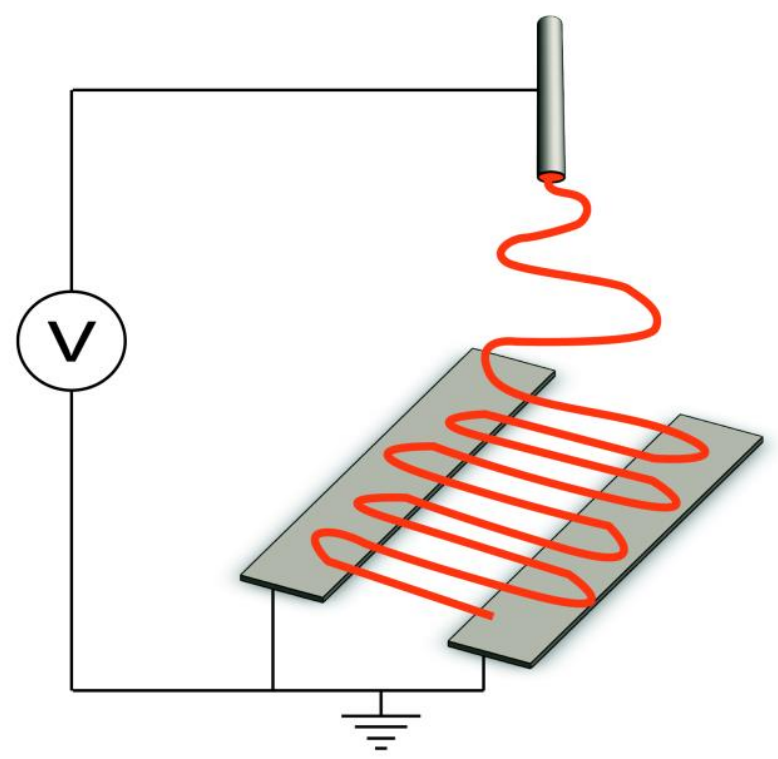

Figure 1. Schematic diagram of the electrospinning process.

\section{Experimental section}

Preparation of the precursor: I prepared a solution of $0.8 \mathrm{M}$ zinc acetate dihydrate (Merck EMSURE ${ }^{\circledR}$ ACS) in a $1: 1 \mathrm{vol} / \mathrm{vol}$ ethanol:water mixture. The solution was heated to $70^{\circ} \mathrm{C}$ for complete dissolution. Then, I added $150 \mathrm{mg} / \mathrm{ml}$ of polyvinyl pyrrolidone (K90, Sigma Aldrich) (PVP) to the solution and left it stirring for $2 \mathrm{~h}$. 
Electrospinning process: Electrospinning was performed on a homemade setup consisting of a spinneret grounded through a high voltage supply (15 kV) to two metallic collector electrodes forming a gap. A schematic representation of the setup is shown in supporting information figure S2. The distance from the spinneret to the ground electrode was $15 \mathrm{~cm}$. The spinneret had an inner diameter of $0.8 \mathrm{~mm}$. The relative humidity and temperature were kept constant at $30 \%$ and $25^{\circ} \mathrm{C}$, respectively. I studied the influence of gap width and deposition time on the alignment and packing of the lines while the flow rate was kept at $0.25 \mathrm{ml} / \mathrm{h}$. I varied the gap between 2.0 and $7.5 \mathrm{~cm}$ and the deposition time between single pulse spinning (spinning time $<0.5 \mathrm{~s}$, termed " $0 \mathrm{~s}$ " in the text) and $15 \mathrm{~s}$. To form flat ribbons, the solution was spun at $70^{\circ} \mathrm{C}$, whereas rounded wires were obtained at room temperature. The samples were annealed in a convection oven at $500^{\circ} \mathrm{C}$ in air for $5 \mathrm{~h}$, using a heating/cooling rate of $5^{\circ} \mathrm{C} / \mathrm{min}$.

Characterization: Scanning Electron Microscopy (SEM) imaging was performed with a Zeiss Merlin HR. Atomic Force Microscopy (AFM) scans were done on a Bruker Icon equipped with a ScanAsyst-Air tip. The imaging of large area arrays of lines was done with a Nikon Eclipse ME600 optical microscope.

Statistical Analysis: The Analysis of variance or ANOVA test is a method used to calculate the difference between more than 2 independent groups of data with normal distribution. The test provides information on the extent to which the data groups have statistically independent results, expressed in terms of the parameter $p$. Smaller values, i.e., $p<0.001$, indicate statistical differences with a large degree of certainty. The ANOVA test was performed using GraphPad.

Device Fabrication: Electrical contacts were defined on a Si substrate with a thermally grown $\mathrm{SiO}_{2}$ layer of $285 \mathrm{~nm}$ thickness by standard photolithography 
using a mask aligner (EVG 620 Mask Aligner), followed by metallization by e-beam evaporation ( $5 \mathrm{~nm} \mathrm{Ti} / 65 \mathrm{~nm}$ Au at a rate of $0.01 \mathrm{~nm} / \mathrm{s}$ ) and lift-off.

Electrical transport and optical measurements: Electrical measurements were conducted in a home built probe station under vacuum conditions $\left(<10^{-5}\right.$ Torr). A Keithley 2643B source meter was used for applying source-drain bias and drain current measurement. A Keithely 2400 source meter was used to apply the voltage. The measurements were performed with an applied gate voltage ranging from -60 to $+60 \mathrm{~V}$. To estimate the field effect mobility I used the formula $\mu=$ $\left[d I_{\mathrm{ds}} / d V_{\mathrm{g}}\right] \cdot\left[\left(L / W C_{\mathrm{i}} V_{\mathrm{ds}}\right)\right.$, where $L$ is the nanowire length and $W$ is the channel width calculated by summing up all nanowire diameters. The capacitance/unit area $C_{\mathrm{i}}$ of the thermally grown $\mathrm{SiO}_{2}$ gate oxide was estimated to be $1.15 \cdot 10^{-4} \mathrm{~F} / \mathrm{m}^{2}$, from $C_{\mathrm{i}}=$ $\varepsilon_{0} \varepsilon_{\mathrm{r}} / d$, where $\varepsilon_{0}$ is the permittivity of vacuum, $\varepsilon_{\mathrm{r}}=3.9$ the relative dielectric constant of $\mathrm{SiO}_{2}$, and $d=300 \mathrm{~nm}$. The optical measurement was performed in the same system by illuminating the device with a UV laser $(405 \mathrm{~nm})$ with a power density of $2.5 \mathrm{~mW} / \mathrm{cm}^{2}$.

\section{Results and discussion}

Our method yielded highly aligned nanowires with a length of $4 \mathrm{~cm}$ over a large area of $4 \times 4 \mathrm{~cm}^{2}$ within a processing of time of $15 \mathrm{~s}$. I used two parallel conductive plates with an insulating gap in between as collector in my experiments, so that the wires became aligned by the imposed electric field and electrostatic wire-wire repulsion [1-4]. I placed the substrates in the electrode gap so that the nanowire arrays would be deposited on them. My study demonstrates that the spacing between metal oxide nanowires can be controlled by varying the spinning time and the gap length between the two collector electrodes. I used $n$-type semiconducting zinc oxide $(\mathrm{ZnO})$ as a model system. However, the method can be extended to almost any metal oxide composition that can be made via an 
electrospinning process. I fabricated a large area field effect transistor (FET) and a UV detector array by aligned electrospinning on top of device substrates with prefabricated electrode arrays. Alternatively, the use of top electrodes which could be fabricated easily on top of nanowire arrays via photolithographic processing is demonstrated.

The electrospinning process described above resulted in large area deposition of highly aligned $\mathrm{ZnO}$ nanowires. The dark field optical microscope image in Figure $2 \mathrm{a}$ exemplifies this. The reflection of a highly aligned array of wires can be seen. I formed arrays of wires of up to $4 \mathrm{~cm}$ in length and width on top of silicon substrates with thermally grown oxide layer of a thickness of $300 \mathrm{~nm}$. To the best of my knowledge, this is the first time that such long fibers and large areas of highly aligned inorganic nanowire arrays are reported.

In general, electrospinning yields nanowires with a circular cross section. When the precursor dried slowly at room temperature after electrospinning, nanowires with a rounded cross section were indeed formed (Figure 2B). After evaporation of the solvents from the fibers, the substrates were annealed at $500{ }^{\circ} \mathrm{C}$ to remove the polymeric matrix and crystallize the $\mathrm{ZnO}$ phase. The $\mathrm{ZnO}$ nanowires formed by this process had an average width of $200 \pm 32 \mathrm{~nm}$ and a height of about $90 \mathrm{~nm}$ (Figure 2D, E and F). Although the cross-section was not completely circular, it can be seen that the solution at room temperature yielded more rounded fibers than the use of a warm solution (Figure 2C). The flattened fibers were formed by spinning the solution at $70{ }^{\circ} \mathrm{C}$. They had a width of $870 \pm 16 \mathrm{~nm}$ and a height of 18 $\pm 3 \mathrm{~nm}$ (Figure $2 \mathrm{D}, \mathrm{E}$ and $\mathrm{F}$ ). The formation of polymeric ribbons by electrospinning has been reported as a consequence of fast drying [16], which may also be the case here. It was explained by the rapid formation of a solid skin around the wet core of the fibers/wires. Eventually the wires collapse into a ribbon shape upon further drying and shrinkage of their inner parts [17-19]. 
Flattened wires may actually be beneficial for certain applications, such as sensing, because of the increased surface to volume ratio.

In order to investigate the control over the nanowire packing density, a matrix of experiments was designed in which the gap width and deposition time were varied. The results are shown in Figure 3. The gap width has a larger effect on nanowire spacing than the deposition time. When different depositions times between 0 (single pulse with duration $<0.5 \mathrm{~s}$ ) and $15 \mathrm{~s}$ were compared, the nanowire packings in samples made with gap widths of 2 and $4 \mathrm{~cm}$ were the same within experimental error (Figure 3a). The average nanowire spacing was $4.5 \mu \mathrm{m}$. In these short experiments, the deposition time had a significant influence on nanowire packing density only at an electrode gap width of $7.5 \mathrm{~cm}$; in that case the wire spacing varied from $239 \mu \mathrm{m}$ at $0 \mathrm{~s}$ to $71 \mu \mathrm{m}$ after $15 \mathrm{~s}$ of deposition.

When samples spun for $15 \mathrm{~s}$ with different gap spacings are compared, the samples made using a $2.0 \mathrm{~cm}$ gap width did not show optimal alignment (Figure $3 \mathrm{~b}$, left). When the gap width was increased to $4.0 \mathrm{~cm}$, alignment was improved (Figure 3b, middle). An average spacing of $71 \mu \mathrm{m}$, while good alignment was kept (Figure $3 b$, right). The result suggests a tipping point beyond which the gap width influences wire packing density. The effect may be associated with the non-linear decrease of the potential at the gap center with increasing gap width, as calculated by Chaurey et al. [15]. Their reported model indicates a potential drop between the center of the gap and the edge of the electrode, which causes the wire alignment. 

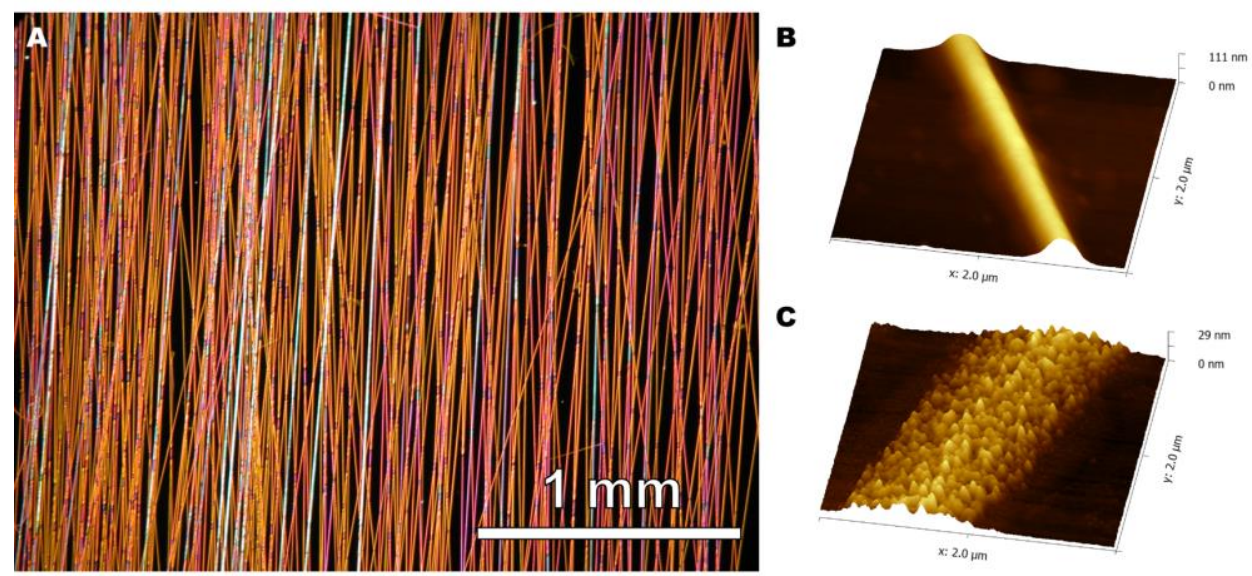

C

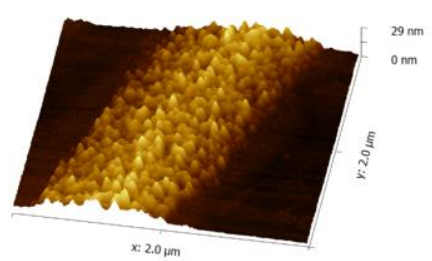

ROUND FIBER

D

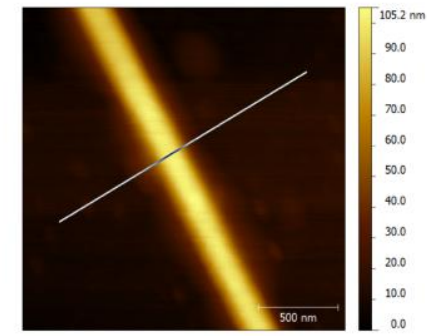

E

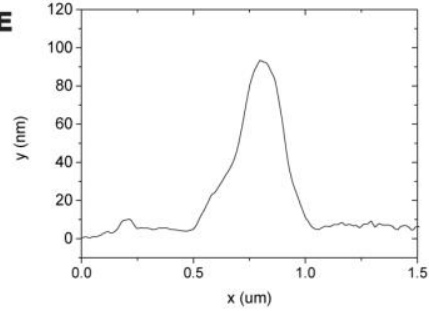

F

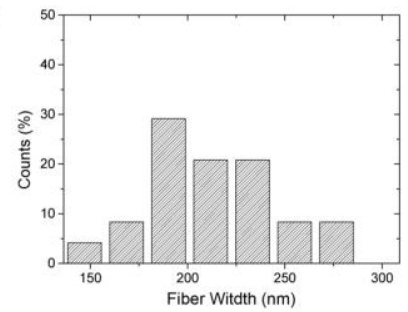

FLAT RIBBON
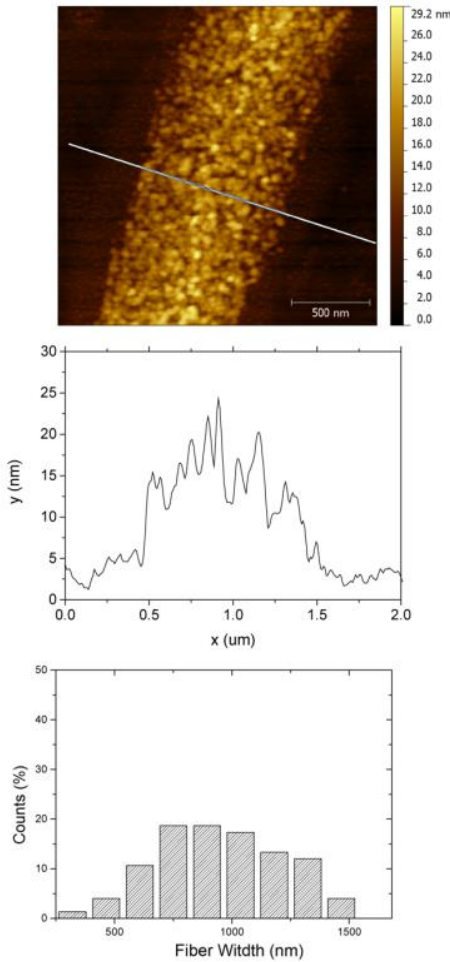

Figure 2. Array of aligned ZnO nanowires. (A) Optical microscope dark field image, in which the reflection of the wires can be seen; (B) 3D AFM topography image of a rounded nanowire; (C) 3D AFM topography of a ribbon-like nanowire; (D) AFM topography scan; (E) Height profile extracted from the AFM scan along the line in (D); (F) Frequency distribution of wire diameter as calculated from SEM images. 
The above results can be understood by considering that wire alignment is electrostatically driven [12]. The electric field lines point towards the metallic electrodes rather than vertically downwards $[10,15]$, i.e. a horizontal electrostatic force component is present, especially in and just above the gap. The magnitude of the horizontal component increases with gap width, resulting in improved alignment for wider gaps. The horizontal electrostatic force drags fibers to the electrodes, thus reducing the chance of depositing fibers in the gap. The packing density is therefore a direct measure of the nanowire deposition rate over the gap. The deposition rate is much higher at short gap widths, since the horizontal component of the electric field towards the electrode is much higher and allows the fibers to be deposited on the gap. The influence of long deposition times can only be appreciated at large gap distances since the deposition rate is much smaller than at short gap distances.

To demonstrate the practical applicability of ordered electrospun $\mathrm{ZnO}$ nanowires I fabricated field effect transistor (FET) arrays and ultraviolet (UV) detector arrays. Aligned nanowires were electrospun on top of heavily doped Si substrates with a thermally grown oxide layer of $300 \mathrm{~nm}$. I used both top and bottom contact source and drain electrode arrays. For bottom contact devices, I fabricated an array of Ti $(2 \mathrm{~nm}) / \mathrm{Au}(60 \mathrm{~nm})$ source-drain electrodes by a photolithography and lift-off process. Then nanowires were electrospun aligned across the electrode arrays, followed by annealing at $500{ }^{\circ} \mathrm{C}$ for $2 \mathrm{~h}$ in air to crystallize $\mathrm{ZnO}$ and obtain device arrays. I have used the rounded nanowires for the fabrication of device arrays. The rounded nanowires had closer packed grains compared to the flat ribbons, which yielded semiconducting nanowires. The flat ribbons did not show 


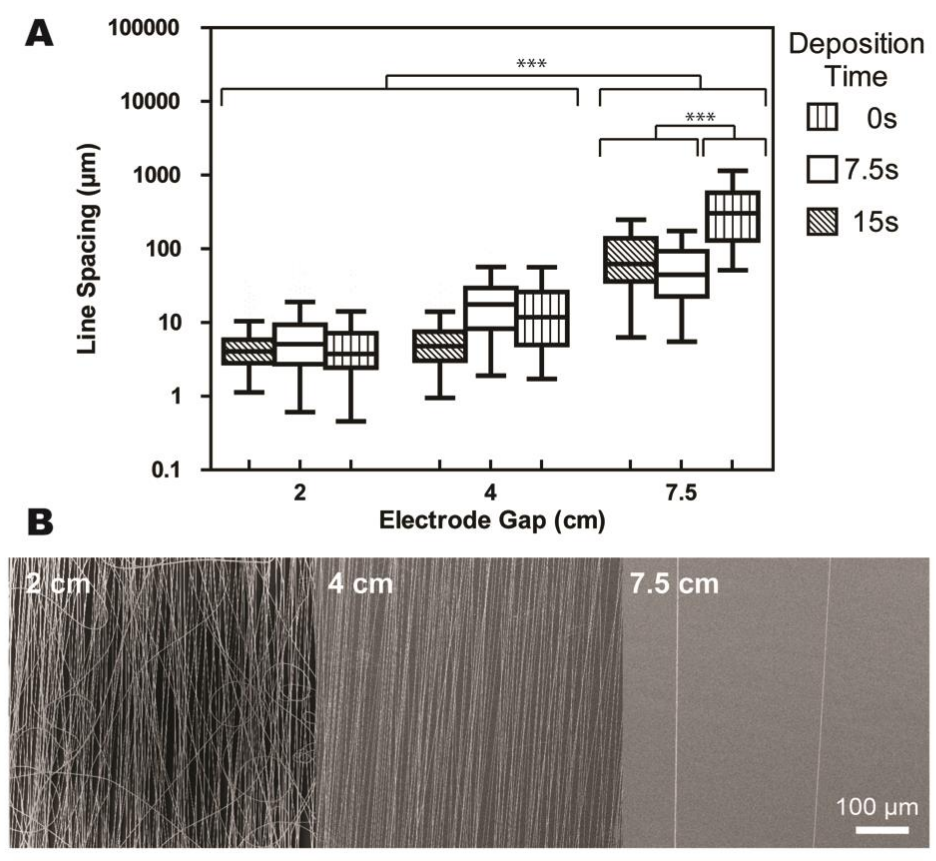

Figure 3. Nanowire spacing. (A) Statistical analysis of samples prepared with different electrode gaps and deposition times. The star signs shows the statistical significance of the difference between samples as calculated by ANOVA tests. The number of stars is a measure of the statistical difference: ${ }^{* *}=p<0.001$. When no stars are shown the samples are statistically equal. (B) SEM images of samples prepared with gap widths of $2.0,4.0$ and $7.5 \mathrm{~cm}$ and pulsed ( $<0.5 \mathrm{~s}$ ) deposition.

any field effect in my electrical measurements and behaved as insulating materials, possibly due to the large spacings between the grains which may have resulted in poor charge transfer. For top contact devices I first electrospun the nanowires on top of a $\mathrm{SiO}_{2} / \mathrm{Si}$ substrate, annealed the wires at $500{ }^{\circ} \mathrm{C}$ in air for $2 \mathrm{~h}$ and then fabricated the source-drain electrodes on top by a photolithography process. In both cases, the electrodes had dimensions of 100×100 $\mu^{2}$ and each electrode was separated by $100 \mu \mathrm{m}$ from the next one. An array of FETs was thus obtained in which the metal contacts functioned as source-drain electrodes. The thermal $\mathrm{SiO}_{2}$ layer functioned as the gate dielectric and the $\mathrm{Si}$ substrate functioned as back gate electrode (schematically shown in Figure 4a). An optical 
microscopy image of the array is shown in Figure 4b. A large area optical micrograph of the device is shown in supporting information figure S3. The same device configuration was used for UV detection. These devices had an average of 16-20 nanowires between the electrodes. Figure 4c shows a typical transfer curve, i.e. drain current plotted against gate voltage $\left(I_{d}-V_{g}\right)$ and in Figure $4 d$, I show the output drain current - drain voltage $\left(I_{\mathrm{ds}}-V_{\mathrm{ds}}\right)$ characteristics of a $\mathrm{ZnO}$ nanowire FET with approximately 16-20 nanowires between the source-drain electrodes. The drain current, $l_{\mathrm{d}}$ increased with positive gate voltage showing the $n$-type behavior of the electrospun $\mathrm{ZnO}$ nanowires. The $I_{\mathrm{ds}}-V_{\mathrm{ds}}$ characteristics showed clear modulation with applied gate voltage. The nanowire FETs had an average mobility of $0.018 \mathrm{~cm}^{2} \mathrm{~V}^{-1} \mathrm{~s}^{-1}$ which is comparable to sol-gel processed $\mathrm{ZnO}$ based thin film transistors [21]. Choi et al. [22] have shown that randomly aligned composite $\left(\mathrm{In}_{2} \mathrm{O}_{3}-\mathrm{ZnO}-\mathrm{ZnGa}_{2} \mathrm{O}_{4}\right)$ nanowires can yield high performance FETs with higher field effect mobility $\left(7.04 \mathrm{~cm}^{2}\right)$. I have used a pure phase of $\mathrm{ZnO}$ without any doping, which has intrinsically lower mobility values similar to sol-gel processed films. However, any engineered materials which can be processed by electrospinning can be prepared in the form large area highly aligned nanowires by my technique. 
A
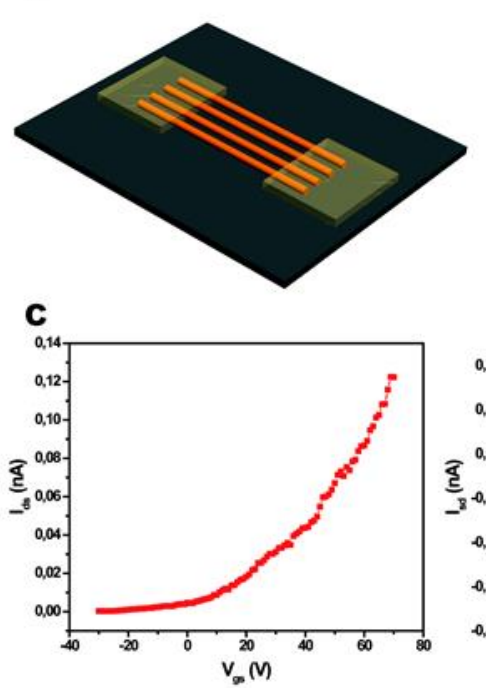
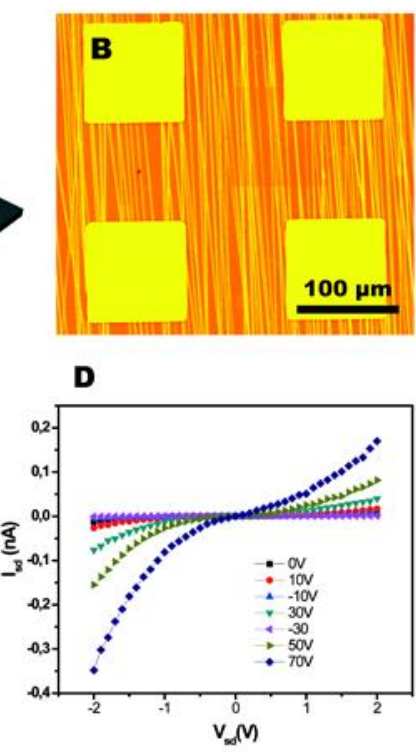

Figure 4. (A) Schematic of electrospun nanowire device. (B) Optical microscopy mage of an electrospun device array. (C) Transfer characteristics of a typical FET (drain current $I_{d s}$ plotted against gate voltage $\mathrm{V}_{G}$ ). The drain voltage is $2 \mathrm{~V}$. (D) I-V characteristics of an electrospun ZnO nanowire transistor. The drain current $I_{d s}$ is plotted against the source-drain bias $V_{d s}$ for different gate voltages.

A photoconductivity study was also performed on this device. As the nanowires were illuminated with a $2.5 \mathrm{~mW} / \mathrm{cm}^{2} 405 \mathrm{~nm}$ laser, it showed a significant photocurrent (Figure 5, red curve) compared with the dark current (black curve). During the measurement, about 20 nanowires with $200 \mathrm{~nm}$ diameter were exposed to the laser, so the effective exposure area is $20 \times 200 \mathrm{~nm} \times 100 \mu \mathrm{m}=$ $400 \mu \mathrm{m}^{2}$. The external quantum efficiency of this device is calculated using the equation $\eta=\frac{I_{p h}}{e} \times \frac{E_{p h}}{p o} \times 100 \%$, where $I_{p h}$ is the difference between current under illumination and dark current, $e$ is the electronic charge, $E_{p h}$ is the photon energy and $p o$ is the incident laser power. The external quantum efficiency was found to be as high as $60 \%$. These device prototypes illustrate the potential 
applicability of aligned electro-spun nanowire devices for low cost electronic and UV-sensing applications.

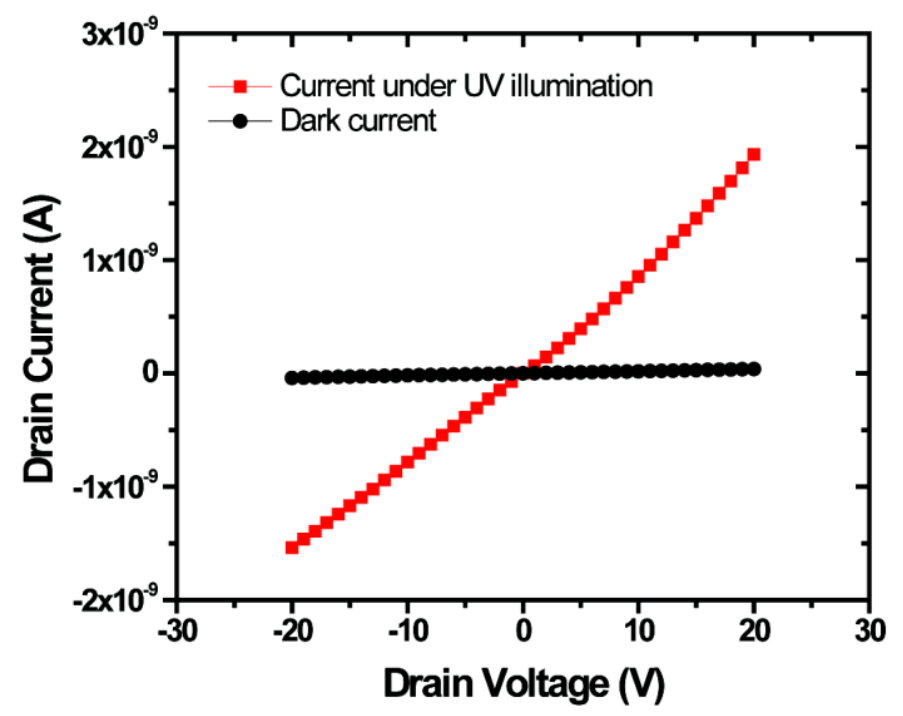

Figure 5. I-V curve of a ZnO nanowire device with and without UV illumination.

\section{Conclusions}

Electrospinning was used to fabricate aligned semiconducting oxide nanowire arrays with $4 \mathrm{~cm}$ long wires and a total surface area of $16 \mathrm{~cm}^{2}$. The nanowire spacing can be controlled by controlling the size of the gap between the electrodes, and to a lesser extent by the deposition time. Aligned nanowire arrays by electrospinning can be used to fabricate low cost large area devices, with control over the nanowire density. My technique can be extended to many other materials that can be electrospun, such as polymers, organic semiconductors, piezoelectric ceramic nanofibers $\left(\mathrm{Pb}(\mathrm{Zr}, \mathrm{Ti}) \mathrm{O}_{3}, \mathrm{BaTiO}_{3}\right.$, etc.) and other functional oxides (e.g. $\mathrm{SnO}_{2}, \mathrm{CuO}, \mathrm{Fe}_{2} \mathrm{O}_{3}, \mathrm{ZnO}, \mathrm{ZrO}_{2}, \mathrm{MgO}$ ), thus providing a generally applicable platform for nanowire array fabrication for low cost electronic, optoelectronic, sensing and energy devices. 


\section{Supporting information}

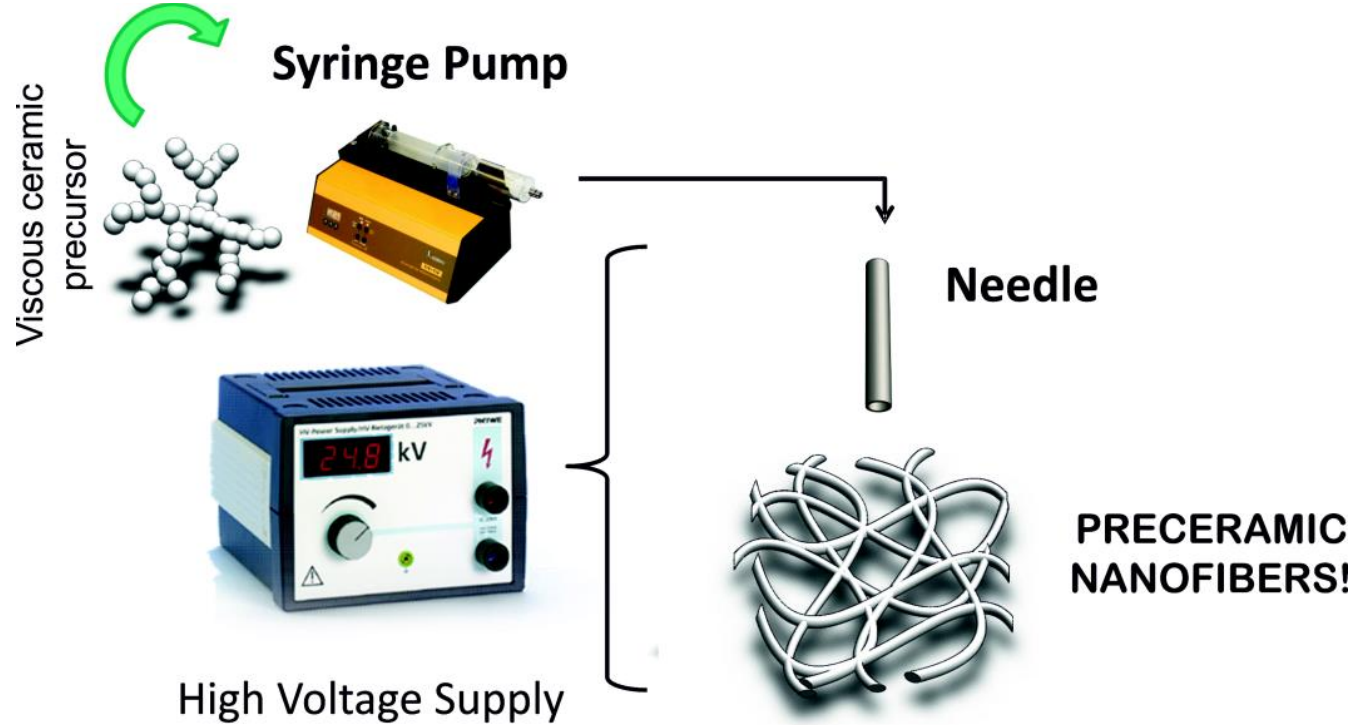

\section{Collector Plate}

Figure S1. Schematic of the electrospinning process.

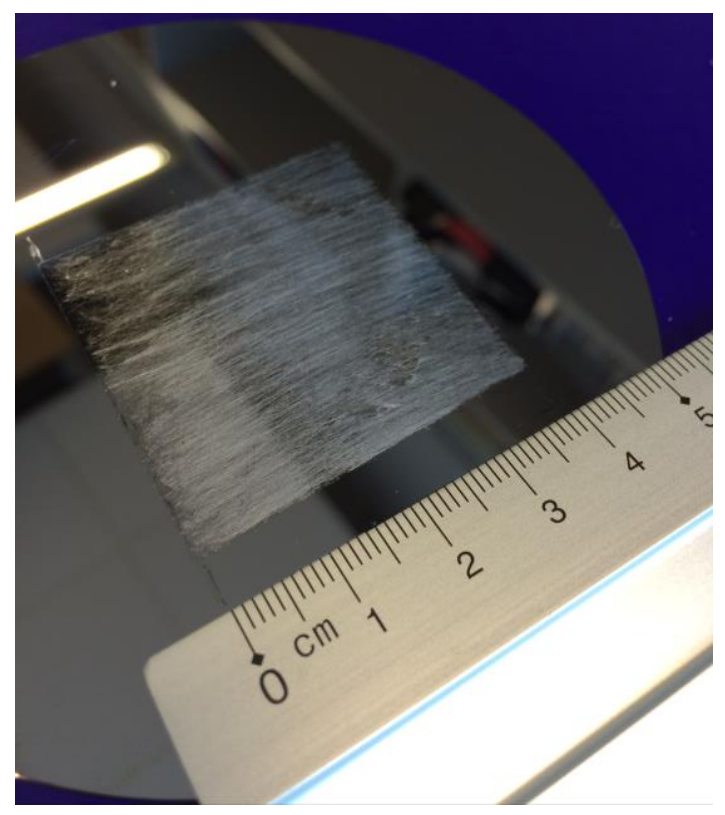

Figure S2. Photograph of aligned ZnO nanofibers. 


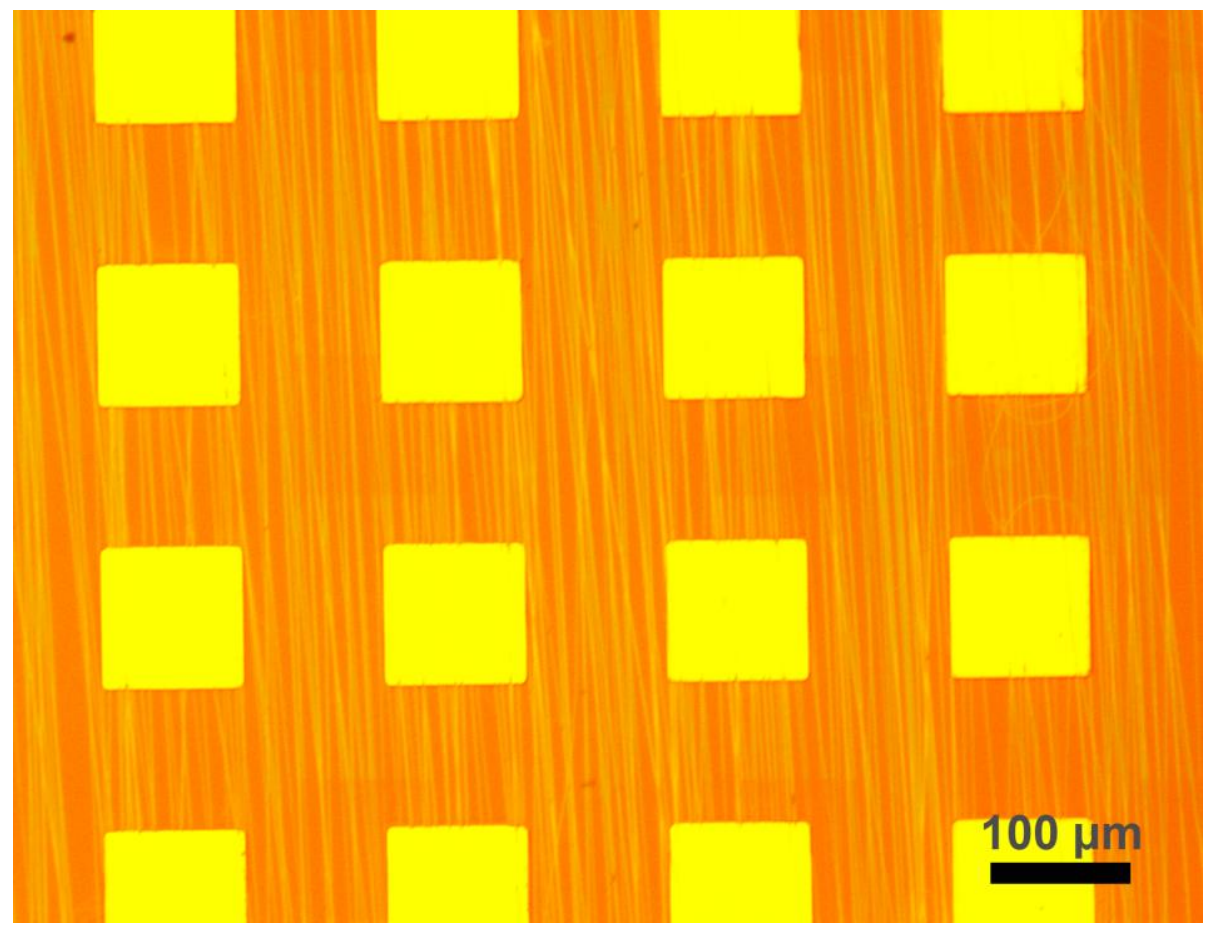

Figure S3. Optical micrograph of ZnO nanowire device array. 


\section{References}

1. Li, W.-J.; Laurencin, C. T.; Caterson, E. J.; Tuan, R. S.; Ko, F. K., Electrospun Nanofibrous Structure: A Novel Scaffold for Tissue Engineering. J. Biomed. Mater. Res. 2002, 60 (4), 613-621.

2. Mai, L.; Xu, L.; Han, C.; Xu, X.; Luo, Y.; Zhao, S.; Zhao, Y., Electrospun Ultralong Hierarchical Vanadium Oxide Nanowires with High Performance for Lithium Ion Batteries. Nano Lett. 2010, 10 (11), 4750-4755.

3. Ding, B.; Wang, M.; Wang, X.; Yu, J.; Sun, G., Electrospun Nanomaterials for Ultrasensitive Sensors. Mater. Today 2010, 13 (11), 16-27.

4. Hongqin, D.; Jian, G.; Hakyong, K.; Doukrae, L., A Novel Method for Preparing Ultra-fine Alumina-borate Oxide Fibres via an Electrospinning Technique. Nanotechnology 2002, 13 (5), 674.

5. Li, D.; Wang, Y.; Xia, Y., Electrospinning of Polymeric and Ceramic Nanofibers as Uniaxially Aligned Arrays. Nano Lett. 2003, 3 (8), 1167-1171.

6. Wu, H.; Lin, D.; Zhang, R.; Pan, W., ZnO Nanofiber Field-Effect Transistor Assembled by Electrospinning. J. Am. Ceram. Soc. 2008, 91 (2), 656-659.

7. Chen, X.; Xu, S.; Yao, N.; Shi, Y., 1.6 V Nanogenerator for Mechanical Energy Harvesting Using PZT Nanofibers. Nano Lett. 2010, 10 (6), 2133-2137.

8. Li, X.; Wang, G.; Wang, X.; Li, X.; Ji, J., Flexible Supercapacitor Based on $\mathrm{MnO} 2$ Nanoparticles via Electrospinning. J. Mater. Chem. A 2013, 1 (35), 1010310106.

9. Zhu, Z.; Zhang, L.; Howe, J. Y.; Liao, Y.; Speidel, J. T.; Smith, S.; Fong, H., Aligned Electrospun ZnO Nanofibers for Simple and Sensitive Ultraviolet Nanosensors. Chem. Commun. 2009, (18), 2568-2570.

10. Lihua, L.; Yuris, A. D., Analysis of the Effects of the Residual Charge and Gap Size on Electrospun Nanofiber Alignment in a Gap Method. Nanotechnology 2008, 19 (35), 355307.

11. Li, D.; Xia, Y., Electrospinning of Nanofibers: Reinventing the Wheel? Adv. Mater. 2004, 16 (14), 1151-1170.

12. Teo, W. E.; Ramakrishna, S., A Review on Electrospinning Design and Nanofibre Assemblies. Nanotechnology 2006, 17 (14), R89.

13. Liu, X.; Gu, L.; Zhang, Q.; Wu, J.; Long, Y.; Fan, Z., All-Printable Band-Edge Modulated ZnO Nanowire Photodetectors with Ultra-High Detectivity. Nat. Commun. 2014, 5, 4007. 
14. Zheng, Z.; Gan, L.; Li, H.; Ma, Y.; Bando, Y.; Golberg, D.; Zhai, T., A Fully Transparent and Flexible Ultraviolet-Visible Photodetector Based on Controlled Electrospun ZnO-CdO Heterojunction Nanofiber Arrays. Adv. Funct. Mater. 2015, 25 (37), 5885-5894.

15. Chaurey, V.; Chiang, P.-C.; Polanco, C.; Su, Y.-H.; Chou, C.-F.; Swami, N. S., Interplay of Electrical Forces for Alignment of Sub-100 nm Electrospun Nanofibers on Insulator Gap Collectors. Langmuir 2010, 26 (24), 1902219026.

16. Fong, H.; Liu, W.; Wang, C.-S.; Vaia, R. A., Generation of Electrospun Fibers of Nylon 6 and Nylon 6-montmorillonite Nanocomposite. Polymer 2002, 43 (3), 775-780.

17. Torres-Giner, S.; Gimenez, E.; Lagaron, J. M., Characterization of the Morphology and Thermal Properties of Zein Prolamine Nanostructures Obtained by Electrospinning. Food Hydrocolloids 2008, 22 (4), 601-614.

18. Koombhongse, S.; Liu, W.; Reneker, D. H., Flat Polymer Ribbons and Other Shapes by Electrospinning. J. Polym. Sci., Part B: Polym. Phys. 2001, 39 (21), 2598-2606.

19. Selling, G. W.; Biswas, A.; Patel, A.; Walls, D. J.; Dunlap, C.; Wei, Y., Impact of Solvent on Electrospinning of Zein and Analysis of Resulting Fibers. Macromol. Chem. Phys. 2007, 208 (9), 1002-1010.

20. Huang, L.; McMillan, R. A.; Apkarian, R. P.; Pourdeyhimi, B.; Conticello, V. P.; Chaikof, E. L., Generation of Synthetic Elastin-Mimetic Small Diameter Fibers and Fiber Networks. Macromolecules 2000, 33 (8), 2989-2997.

21. Fleischhaker, F.; Wloka, V.; Hennig, I., ZnO Based Field-Effect Transistors (FETs): Solution-Processable at Low Temperatures on Flexible Substrates. J. Mater. Chem. 2010, 20 (32), 6622-6625.

22. Choi, S.-H.; Jang, B.-H.; Park, J.-S.; Demadrille, R.; Tuller, H. L.; Kim, I.-D., Low Voltage Operating Field Effect Transistors with Composite In2O3-ZnOZnGa2O4 Nanofiber Network as Active Channel Layer. ACS Nano 2014, 8 (3), 2318-2327. 


\section{Chapter 6}

\section{Electrospun fibers as sacrificial templates to form submicron channel porosity in 3YSZ with control over pore shape, size and NiO catalyst deposition}

\section{Abstract}

Electrospun polymeric fibers were used as sacrificial templates to form nanochannel porosity in yttrium stabilized zirconia solid electrolytes for solids oxide fuel cells (SOFC). The pore diameter was varied from 100 to $160 \mathrm{~nm}$, and the directionality of the nanochannels could be controlled. Moreover, when these fibers were loaded with a nickel precursor, nickel oxide was locally deposited on the inner walls of the pores in a controlled manner. The catalytic activity of the nickel oxide containing ceramic with structured porosity was superior to the commercial reference material due to the more optimal distribution of the catalyst. This technique provides a simple yet elegant method to form functionalized porous ceramics. 


\section{Introduction}

Porous ceramics have gained interest due to their enhanced properties and applicability in the fields of energy, chemical engineering, electronics and biomedicine [1-3]. Porous materials are typically divided into 3 categories, depending on the pore diameter $d$ : microporous $(d<2 n m)$, mesoporous $(2 \mathrm{~nm}<$ $\mathrm{d}<50 \mathrm{~nm}$ ) and macroporous ( $\mathrm{d}>50 \mathrm{~nm}$ ) [2]. Macroporous ceramics have been useful as Solid Oxide Fuel Cell (SOFC) electrodes, membranes for water and gas purification, bone regeneration scaffolds, gas sensing devices and photonics [1-4].

The desired microstructure of these materials has interconnected porosity, narrow size distribution of the pore diameters and a high relative porosity [5]. Classic preparation methods are the replica technique, the sacrificial template technique and direct foaming, all leading to a minimum pore size of $1 \mu \mathrm{m}$ and/or a maximum porosity of $96 \%$ [2].

Little work has been reported that focuses on narrowing the distribution of the sub-micron pore sizes. The use of polystyrene beads has been reported to lead to sub-micron porosity in titania and zirconia [6]. Also, the self-assembly using emulsions shows promising results in forming well organized sub-micron porosity in silica and titania [7]. However, both techniques are technically complicated and not available on industrial scale. Moreover, high porosities are typically achieved by adding large relative amounts of pore formers, which also results in larger pore sizes [8].

Certain applications require a second active phase dispersed homogeneously in a porous matrix, for instance, heterogeneous metal or metal oxide catalysts for SOFC and reactive membranes. The classical methods do not control the catalyst deposition process or the precise location well. The catalyst may end up in the 
bulk of the material, unable to catalyze the reaction with a gas, leading to an inactive catalyst [9], and in some cases even to damage of the integrity of the material [10].

An example is the SOFC anode, which consists of a cermet between nickel oxide and yttria-stabilized zirconia (YSZ) [11]. During operation, the reduced nickel phase acts as a catalyst for the oxidation of fuels with ionic oxygen transported through the electrolyte, leading to formation of electrons [1].

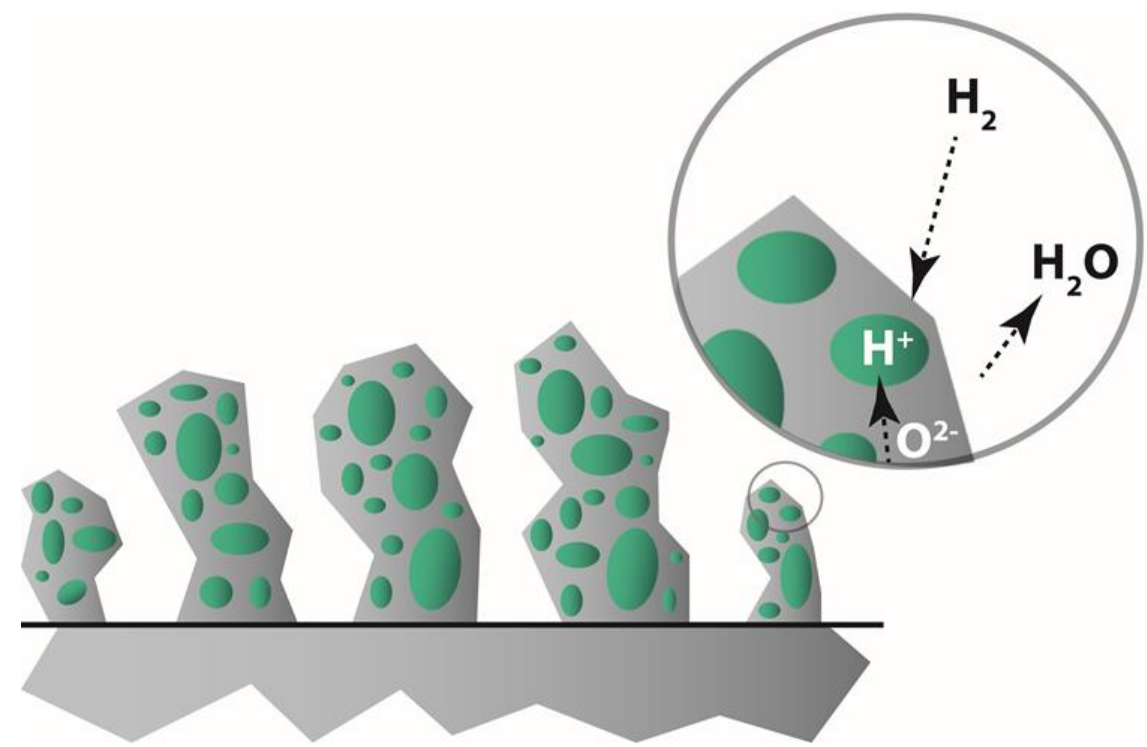

Figure 1. Schematic of the working principle of a SOFC anode. The YSZ phase (grey) transports ionic oxygen towards the surface, where it reaches the nickel phase (green). The nickel phase adsorbs hydrogen from the gas phase, transforms it into $\mathrm{H}^{+}$and catalyzes the reaction between the proton and ionic oxygen into water.

Figure 1 shows this process schematically. The nickel oxide phase undergoes cycles of reduction and oxidation during operation, resulting in a volumetric contraction and expansion. This phenomenon results in internal stresses in the material, which may cause fracture [10]. Moreover, when hydrocarbons are used then carbon is deposited on the nickel phase and may deactivate it $[10,11]$. 
If the catalyst would be located only on the surface of the pore, the efficiency would be higher. In that case, only a low amount of nickel would be required. I may also expect that the volumetric expansion and reduction would not affect the composite's integrity as much, because the nickel oxide phase would be able to expand freely into the voids of the pores.

To fabricate a porous active ceramic material with monodisperse pores of low tortuosity, I used electrospun polymer fibers as sacrificial templates. Electrospinning is a simple technique to fabricate nanofibers, and can be scaled up to industrial scale [12]. Briefly, the technique consists of pumping a polymeric solution through a spinneret. This spinneret is connected to a grounded collector plate via a high voltage supply. As a result, the solution is electrified and forms a jet that is drawn towards the counter electrode and results in micro- and nanofibers that are dried and collected on the ground plate $[13,14]$. A schematic of the electrospinning process is presented in Figure 2.

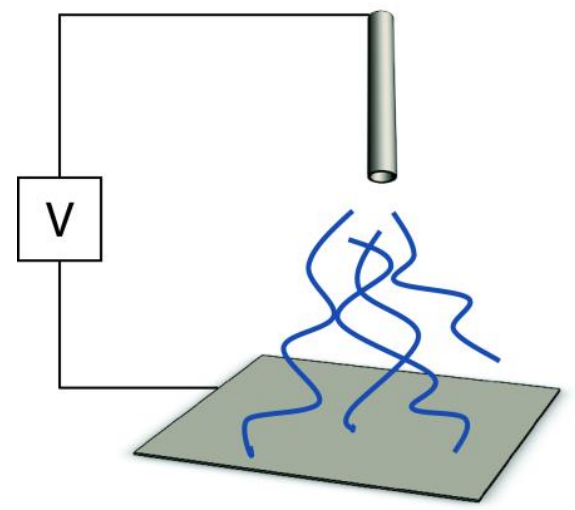

Figure 2. Schematic of electrospinning setup. A spinneret needle carrying a precursor solution and a grounded collector plate are connected via a high voltage supply.

In the present study, electrospun fibers were employed as sacrificial templates to form submicron-channel porosity in an otherwise dense YSZ ceramic material. The 
finely dispersed deposition of nickel oxide was locally controlled on the inner surface of the pores. As Figure 3 illustrates schematically, electrospun polymeric fibers loaded with a nickel precursor were used as templates. After spinning and drying, a sol-gel YSZ ceramic precursor solution was infiltrated as a matrix surrounding the nanofibers. When the sample was then annealed to form a crystalline YSZ phase, the polymeric phase of the nanofibers was oxidized completely, thus acting as pore formers. Therefore, YSZ with high porosity in the shape of straight nanochannels of the same diameter was accomplished. The nickel phase present in the polymeric nanofiber was deposited as nickel oxide phase on the inner wall of the submicron channel pores. This preparation method is a much simpler alternative to form sub-micron porosity than the previously reported self-assembly methods $[6,7]$.

The goal of this study was to prove the principle of formation of nanochannel porosity in ceramics and control over catalyst deposition. The proposed process leads to a highly efficient catalytic material with control over the final microstructure. Ni-YSZ cermets for SOFC anodes were used as model systems to illustrate the method. 
A

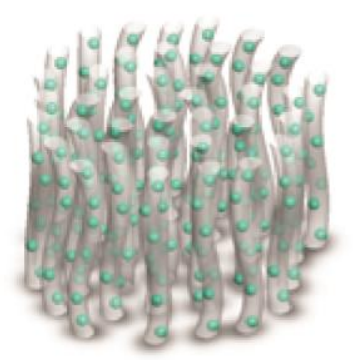

C

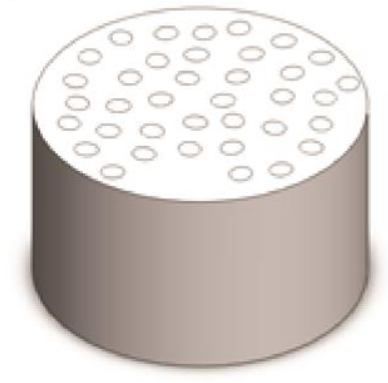

B

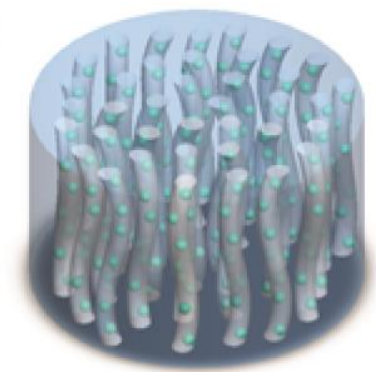

D

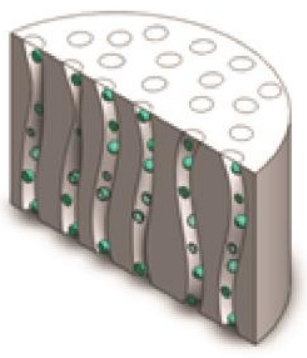

Figure 3. Schematic of the templating method. (A) electrospun polymeric fibers loaded with nickel; (B) infiltration of sol-gel precursor into the fiber matrix; (C) thermal annealing of the material to form a crystalline ceramic phase, leading to (D) formation of a film with submicron straightchannel porosity and local nickel deposition on the internal pore walls after burn-out during thermal annealing.

\section{Materials}

PolyActive (PolyVation BV) is a copolymer formed from $45 \mathrm{wt} \%$ polyethylene oxide terephthalate (MW $300 \mathrm{~g} / \mathrm{mol}$ ) and $55 \mathrm{wt} \%$ polybutylene terephthalate. Chloroform was purchased from Sigma Aldrich and 1,1,1,3,3,3-hexafluoro-2propanol (HFIP) from Biosolve. Nickel stearate was obtained from Sigma Aldrich, 
nickel nitrate hexahydrate from Merck and nickel oxide nanoparticles $(<50 \mathrm{~nm}$ particle size, $99.8 \%$ purity) from Sigma Aldrich.

\section{Methods}

The polymer solution for electrospinning consisted of $200 \mathrm{mg} / \mathrm{ml}$ PolyActive in a $30: 70 \mathrm{vol} / \mathrm{vol}$ solution of chloroform : HFIP. To obtain nickel-free fibers, the solution was spun as such. For nickel containing fibers, three strategies were used: solution, dispersion and suspension. The solution route employed a solution consisting of $100 \mathrm{mg} / \mathrm{ml}$ nickel stearate dissolved in the polymer solution.

In the dispersion route, nickel nitrate was dissolved in ethanol at a concentration of $625 \mathrm{mg} / \mathrm{ml}$. Then octylamine was added to the solution, in a molar ratio of octylamine : nickel of $6: 1$, to avoid the phase separation of nickel nitrate in ethanol solution from the chloroform-rich phase. Then, the nickel and PolyActive containing solutions were mixed in a $30: 70 \mathrm{vol} / \mathrm{vol}$ ratio, respectively. For the suspension route, nickel oxide nanoparticles were mixed with the PolyActive solution to a concentration of $20 \mathrm{mg} / \mathrm{ml} \mathrm{NiO}$ and the solution was left stirring for $10 \mathrm{~min}$. The viscosity of the polymer solution itself allowed formation of a suspension that was stable enough for fiber fabrication.

All solutions were spun using a homemade electrospinning setup. The flow rate was set to $1 \mathrm{ml} / \mathrm{h}$, the voltage at $15 \mathrm{kV}$, and the distance from spinneret to collector plate was $20 \mathrm{~cm}$. To align the fibers, the flat collector plate was replaced by a rotating mandrel of $6 \mathrm{~cm}$ diameter rotating at $3000 \mathrm{rpm}$. After spinning, the fibers were removed from the collector and immersed into a YSZ precursor solution. 
The YSZ sol-gel precursor solution was prepared as reported by Veldhuis et al. [15]. Briefly, a $1 \mathrm{M}$ solution of zirconium isoproxoxide in 2-methoxyethanol was made. Separately, $1 \mathrm{M}$ of yttrium acetate was dissolved in acetic acid. Then the zirconium solution, acetic acid, yttrium solution and water were mixed in a volume ratio of 1000:229:69.1:90, resulting in the gross composition $\mathrm{Y}_{0.065} \mathrm{Zr}_{0.935} \mathrm{O}_{1.978}$.

After infiltration of the YSZ precursor into the nanofiber compacts, the fibers were left to dry at room temperature. Then, the material was annealed at $850^{\circ} \mathrm{C}$ in air for $2 \mathrm{~h}$ at heating/cooling rates of $5 \stackrel{\circ}{\circ} / \mathrm{min}$.

The prepared material was imaged with a Zeiss Merlin HR FEG scanning electron microscope (SEM). The nickel distribution was measured by a Noran Systems Six energy dispersion X-ray (EDX) analyzer coupled with a Philips CM300ST transmission electron microscope (TEM). Thermogravimetic analysis (TGA) coupled with differential scanning calorimetry (DSC) was performed using a Netzsch STA 449 F3 Jupiter system. The crystal phase of the prepared materials was studied by X-Ray Diffraction (XRD) in the $2 \theta$ range between 20 and $70^{\circ}$ using a PANAlytical $X^{\prime}$ Pert.

The pore diameter was measured by mercury intrusion and the porosity of the sample by an Archimedes method using a PG503 DeltaRange balance. The samples' weigths were measured in both air and water. The relative density was calculated based on a theoretical density of YSZ of $6.05 \mathrm{~g} / \mathrm{cm}^{3}$.

The active surface area of the nickel phase was determined by hydrogen chemisorption. Prior to chemisorption, the reduction temperature of nickel oxide was measured with in-house built temperature-programmed reduction equipment. The results showed that nickel oxide is fully reduced to nickel before the onset of zirconia reduction at $650{ }^{\circ} \mathrm{C}$. Chemisorption experiments were 
performed using a Micromeritics ChemiSorb 2750 (Pulse Chemisorption system). The sample was pretreated at $650^{\circ} \mathrm{C}$ in hydrogen gas for $1 \mathrm{~h}$. Then, the sample was exposed to $\mathrm{Ar}$ at $650^{\circ} \mathrm{C}$ for $45 \mathrm{~min}$, to remove adsorbed hydrogen, and then cooled down in Ar until room temperature was reached. The chemisorption measurements were performed at room temperature. They were done by pulsing $H_{2}: A r(1: 99 \mathrm{vol} / \mathrm{vol})$ until no further hydrogen was adsorbed by the sample.

The nickel content was measured with an Inductively Coupled Plasma Optical Emission Spectrometer (ICP-OES), Varian Liberty II model, a sequential ICO-OES with a radial plasma. Prior to ICP, the samples were dissolved in concentrated nitric acid $(65 \%)$ at $100^{\circ} \mathrm{C}$ for $24 \mathrm{~h}$ and then diluted 20 times by volume. To calibrate the ICP equipment, reference samples with varying nickel concentrations were prepared using $\mathrm{NiO}$ as the calibrant and measured.

Electrochemical Impedance Spectroscopy was performed on the sample. A flake of the templated material with known dimensions (length $0.2 \mathrm{~cm}$, cross-section $0.28 \times 0.025 \mathrm{~cm}^{2}$ ) was placed on an alumina plate and between two platinum wires as electrodes. The sample was connected to the wires with platinum paint (SPI-Paint). The sample holder was connected to an Autolab PGSTAT128N using separate current lines and voltage probes (pseudo four point measurement). The sample was placed in an oven at $650-850{ }^{\circ} \mathrm{C}$. The environment of the oven was controlled to be similar to the SOFC anode working conditions, i.e. $2.2 \mathrm{~mol} \%$ of water in a gas atmosphere of $91: 9 \mathrm{~mol} / \mathrm{mol} \mathrm{N}_{2}: \mathrm{H}_{2}$. All impedance measurements were performed at an amplitude of $50 \mathrm{mV}$ and a range of frequencies between 1 $\mathrm{MHz}$ and $100 \mathrm{~Hz}$. 


\section{Results and discussion}

The pore formation method was proved by using polymeric fibers without any nickel loading. Figure 4 shows the fabricated polyactive nanofibers and templated YSZ material. The nanofibers formed a highly porous matrix with a morphology typical for electrospun fibers $[13,14]$. The YSZ ceramic material formed the negative replica of the fiber network, yielding a network of submicron channels in a solid phase. Figure $4 \mathrm{~B}$ and $4 \mathrm{C}$ show the front and top views of the submicron channel network, respectively. The 3YSZ crystalline phase was confirmed by XRD (Figure 4D).

Aligned, unidirectional porosity is of great interest, especially for membrane applications, due to the enhanced permeability or capillary rise (ability to drag liquid) [5] in the absence of pore tortuosity. Electrospun fibers can be mechanically aligned via collection on a rotating mandrel (Figure 5 A, B). Such fibers can act as a template to form aligned porosity in the YSZ phase as illustrated in Figure 5 C, D. The pore sizes obtained here are smaller than previously reported ceramics with aligned porosity fabricated by a template method [5]. 

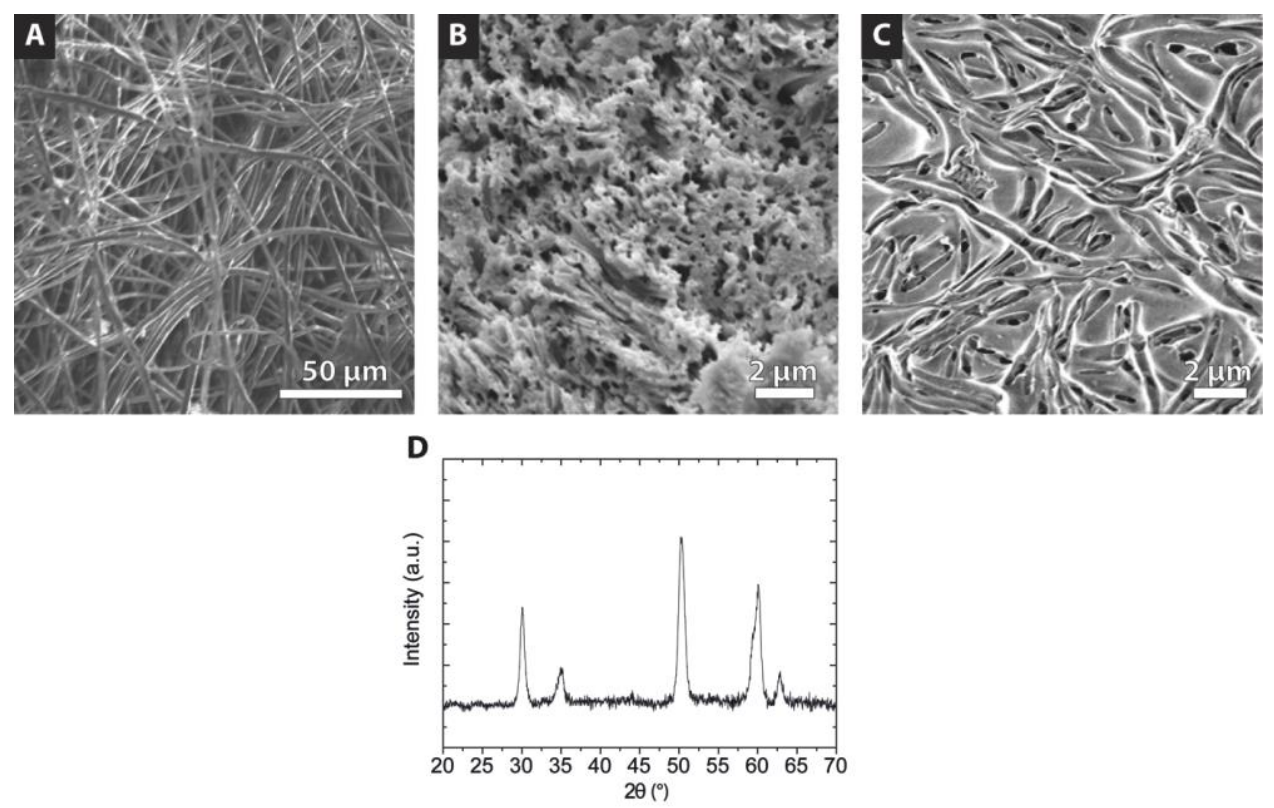

Figure 4. SEM pictures: (A) as prepared electrospun Polyactive nanofibers, (B) cross-section of the templated YSZ material fater themal annealing; (C) top view of the templated YSZ material after thermal annealing. D) XRD Pattern of the material showing 3YSZ phase.

Fibers of three different diameters were prepared to investigate their influence on pore diameter and pore density. The results are presented in Figure 6. In agreement with previous reports, the polymer concentration has a direct influence of the fiber diameter [16-18]. Figure 6A illustrates the linear relationship between fiber diameter and pore diameter. The pore size increased with fiber diameter. I fabricated fibers of $530 \pm 200 \mathrm{~nm}, 1200 \pm 440 \mathrm{~nm}$ and $2000 \pm 600 \mathrm{~nm}$, which resulted in pore diameters of $106 \pm 6 \mathrm{~nm}, 128 \pm 16 \mathrm{~nm}$ and $160 \pm 20 \mathrm{~nm}$, respectively (Figure $6 \mathrm{~A}$ ), after thermal treatment. Such a decrease in pore diameter is attributed to the weight loss of polymer ( $80 \mathrm{wt} \%$ ) at temperatures < $400^{\circ} \mathrm{C}$, followed by crystallization and consequently shrinkage of the porous YSZ phase during sintering at $500^{\circ} \mathrm{C}$ and higher temperatures. The thermogravimetric 

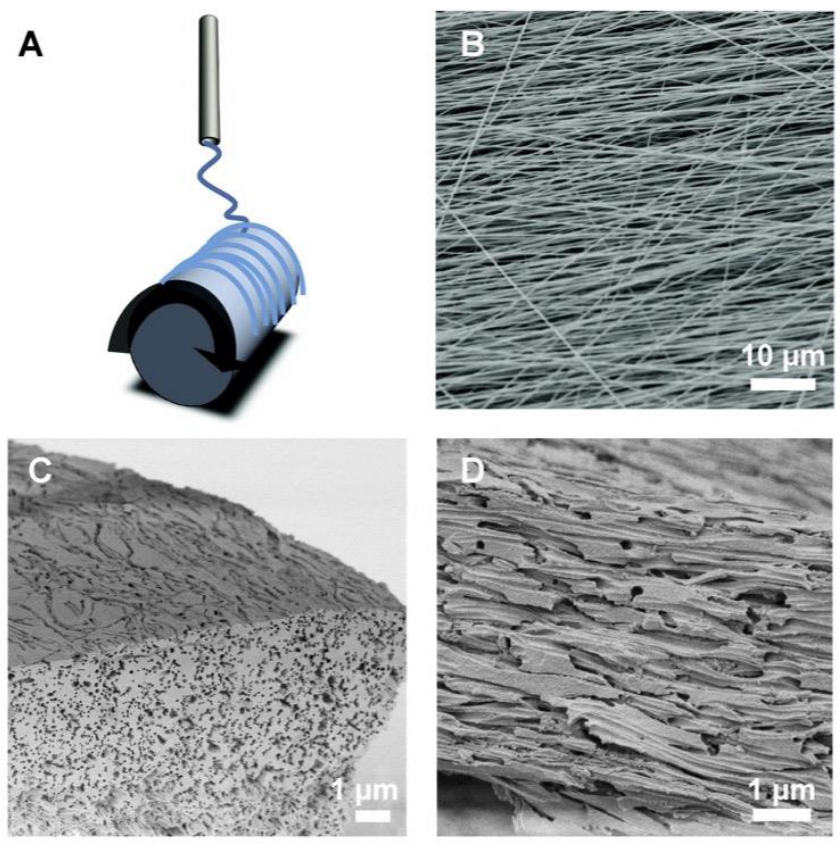

Figure 5. (A) Alignment of fibers on a mandrel directly following electrospinning. (B) aligned electrospun polymeric fibers. (C) SEM image of templated YSZ phase with unidirectional porosity. (D) SEM picture of YSZ phase with unidirectional pores at higher magnification.

analysis presented in Figure $6 \mathrm{~B}$ shows a considerable weight loss in the temperature range $350-450^{\circ} \mathrm{C}$ indicating combustion and decomposition of the organic phases present in the green fibers. The exothermic differential scanning calorimetry signal in Figure $6 \mathrm{~B}$ at $300-450^{\circ} \mathrm{C}$ confirms the oxidative burnout of organics. The DSC signal also displays a peak at $475-500^{\circ} \mathrm{C}$, which is attributed to the crystallization of the 3YSZ phase. Very high porosities of up to $70 \%$ (relative to the density of dense YSZ that is $\sim 6.1 \mathrm{~g} / \mathrm{cm}^{3}$ ) were achieved; the pore diameter did not have a statistically significant influence on total porosity, as determined by statistical analysis of variance (Figure $6 \mathrm{C}$ ). This means that the fiber packing was constant regardless of fiber diameter. The porosity was therefore also constant as it is the negative replica of the fiber mat. 
Having demonstrated the principle of using electrospun fibers as pore forming templates, the polymeric fibers were loaded with nickel-containing precursors to deposit nickel oxide on the surface of the pore. I applied three different strategies to do that: from solution, from a dispersion and from a suspension.
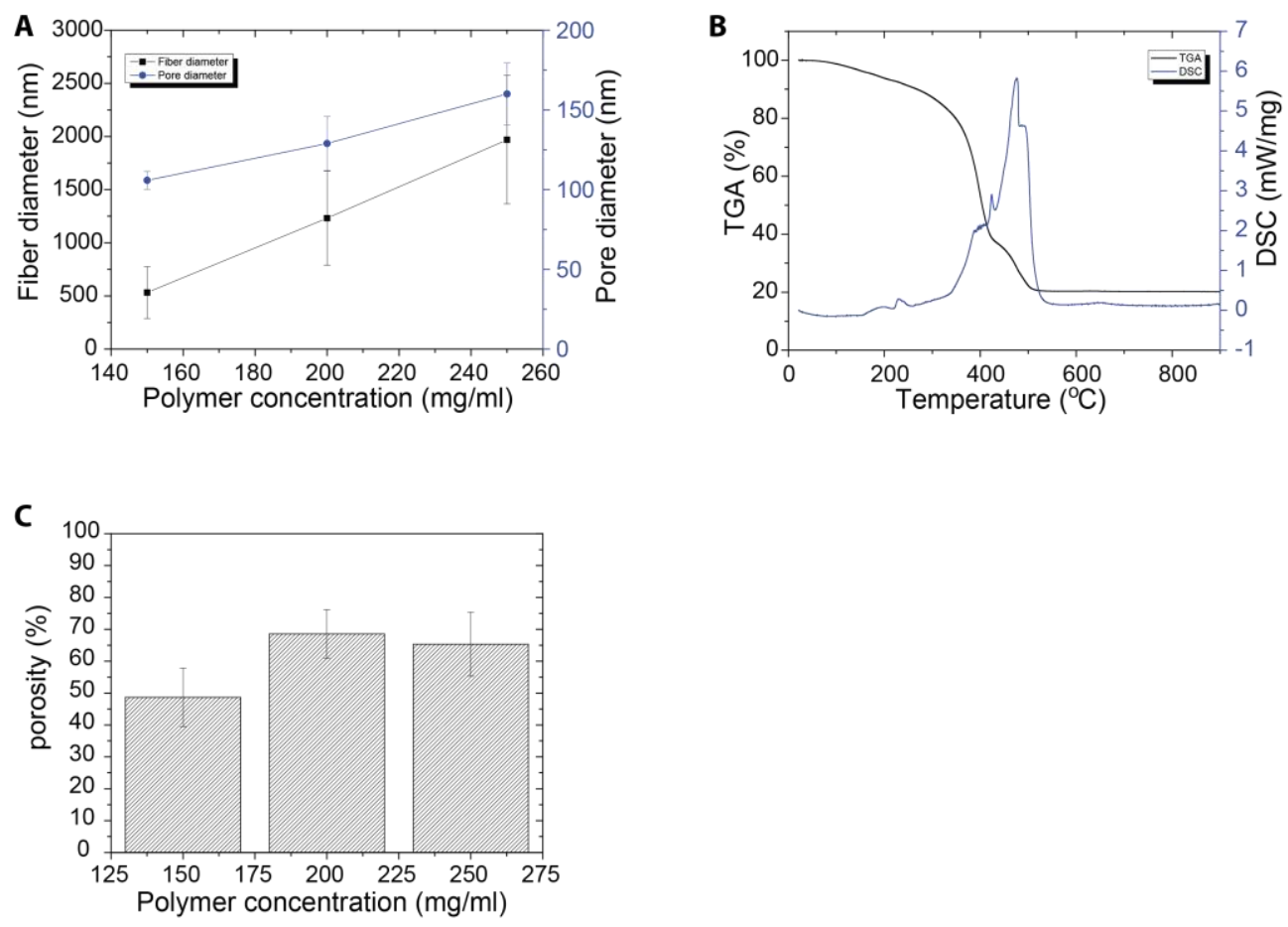

Figure 6. (A) Fiber diameter with varying polymer concentrations and corresponding pore sizes;

(B) TGA/DSC data of the polymeric fibers infiltrated by YSZ sol-gel precursor solution (heating rate $\left.5{ }^{\circ} \mathrm{C} / \mathrm{min}\right)$; (C) porosity of the YSZ materials templated with fibers fabricated from solutions with varying polymer concentration.

After YSZ precursor infiltration in all fibrous network structures and thermal annealing, porous YSZ microstructures loaded with finely dispersed nickel oxide phase were obtained. SEM imaging revealed a change of surface roughness when nickel was added, as presented in Figure 7. The use of bare polymeric fibers resulted in smooth submicron channels (Figure 7A). In contrast, the microchannel surfaces became much rougher when nickel stearate (Figure 7B), nickel nitrate 
(Figure 7C) or nickel oxide nanoparticles (Figure 7D) had been dissolved or dispersed in the polymer solution. The clear microstructural difference with Figure 7A suggests the presence of nickel oxide on the surface of the channels.
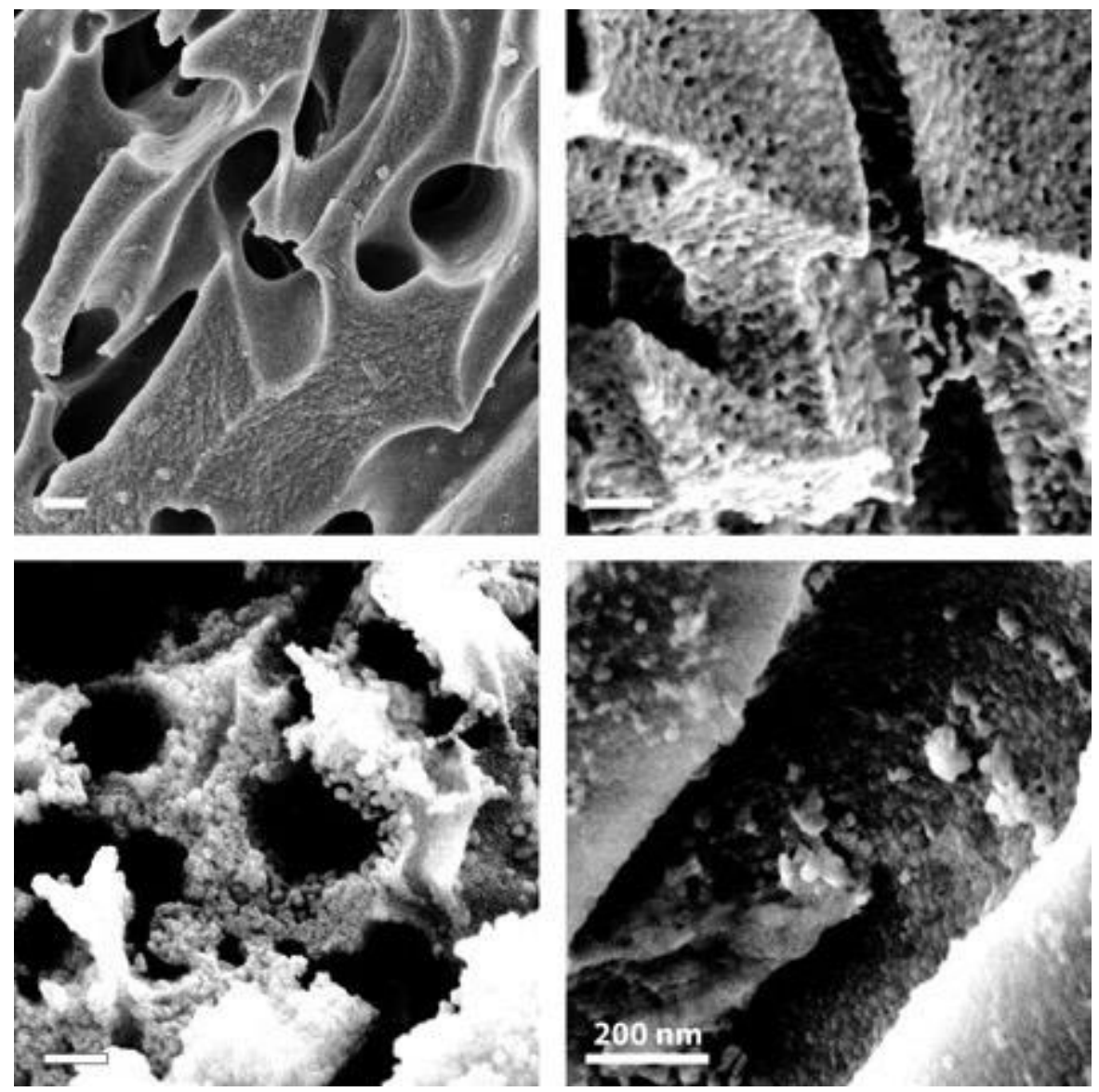

Figure 7. SEM images of YSZ with channel porosity. (A) templated YSZ film made using nickel-free fibers. (B) templated YSZ using fibers loaded with nickel stearate (solution). (C) templated YSZ using fibers loaded with nickel nitrate (dispersion). (D) templated YSZ using fibers loaded with nickel oxide nanoparticles (suspension).

The deposition of nickel on the inner surface of the submicron channels was confirmed by EDX. Point analyses at the cross-section of the material to investigate the location of nickel oxide within the material are shown in Figure 8. 
The images show a pore with the locations where several point analyses at varying distances from the pore wall were done. Nickel stearate (Figure $8 \mathrm{~A}$ ) and nickel nitrate (Figure 8B) samples did not show a significant enrichment of the nickel concentration at the pore wall. In contrast, the nickel oxide nanoparticle sample (Figure $8 \mathrm{C}$ ) shows a much higher concentration of nickel at the pore wall than in the bulk. Local deposition and significant enrichment of the Ni catalyst on the 3YSZ pore wall occurred when the polymeric fibers were loaded with nickel oxide nanoparticles. Nickel salt solutions (stearate dissolved in chloroform) and dispersions (nitrate dispersed in chloroform using octylamine) resulted in a more homogeneous distribution of $\mathrm{Ni}$ in the YSZ matrix, as the elemental point analysis in the bulk in Figure 8 indicate. Moreover, the fibers containing NiO nanoparticles had a higher nickel loading $(1.3 \mathrm{mmol} \mathrm{Ni} / \mathrm{mg}$ polymer) than the dissolved $(0.8$ $\mathrm{mmol} \mathrm{Ni} / \mathrm{mg}$ polymer) and dispersed $(0.8 \mathrm{mmol} \mathrm{Ni} / \mathrm{mg}$ polymer) systems. It is noted that further loading of nickel could not be achieved in the latter two systems as the limit of $\mathrm{Ni}^{2+}$ solubility has been reached.

Composite mixtures of nickel oxide and YSZ form so-called cermets [19]. It is known that nickel tends to diffuse and become incorporated in the YSZ crystal lattice at elevated temperatures. Delaforce et al. reported nickel migration up to $200 \mu \mathrm{m}$ into a YSZ lattice upon solid state reaction after firing at $1450^{\circ} \mathrm{C}$ for $4 \mathrm{~h}$ [20]. Sol-gel precursors are typically much better mixed on atomic scale than atoms from solid-state phases, which allows thermal processing at lower temperatures $[21,22]$. But the distances over which atoms diffuse are then also smaller for similar processing times. This suggests that $\mathrm{Ni}$ ions from the $\mathrm{NiO}$ nanoparticle phase will penetrate less into the 3YSZ phase than at higher sintering temperature. On the other hand, the atomically dispersed nickel ions from stearate and nitrate salts have a higher solubility and mobility in the precursor phase of 3YSZ. The nickel nanoparticle suspensions will therefore form a nickel 
oxide layer on the surface of the pore, whereas the nickel ions from the stearate solution and nitrate dispersion will have been able to diffuse further into the YSZ bulk.
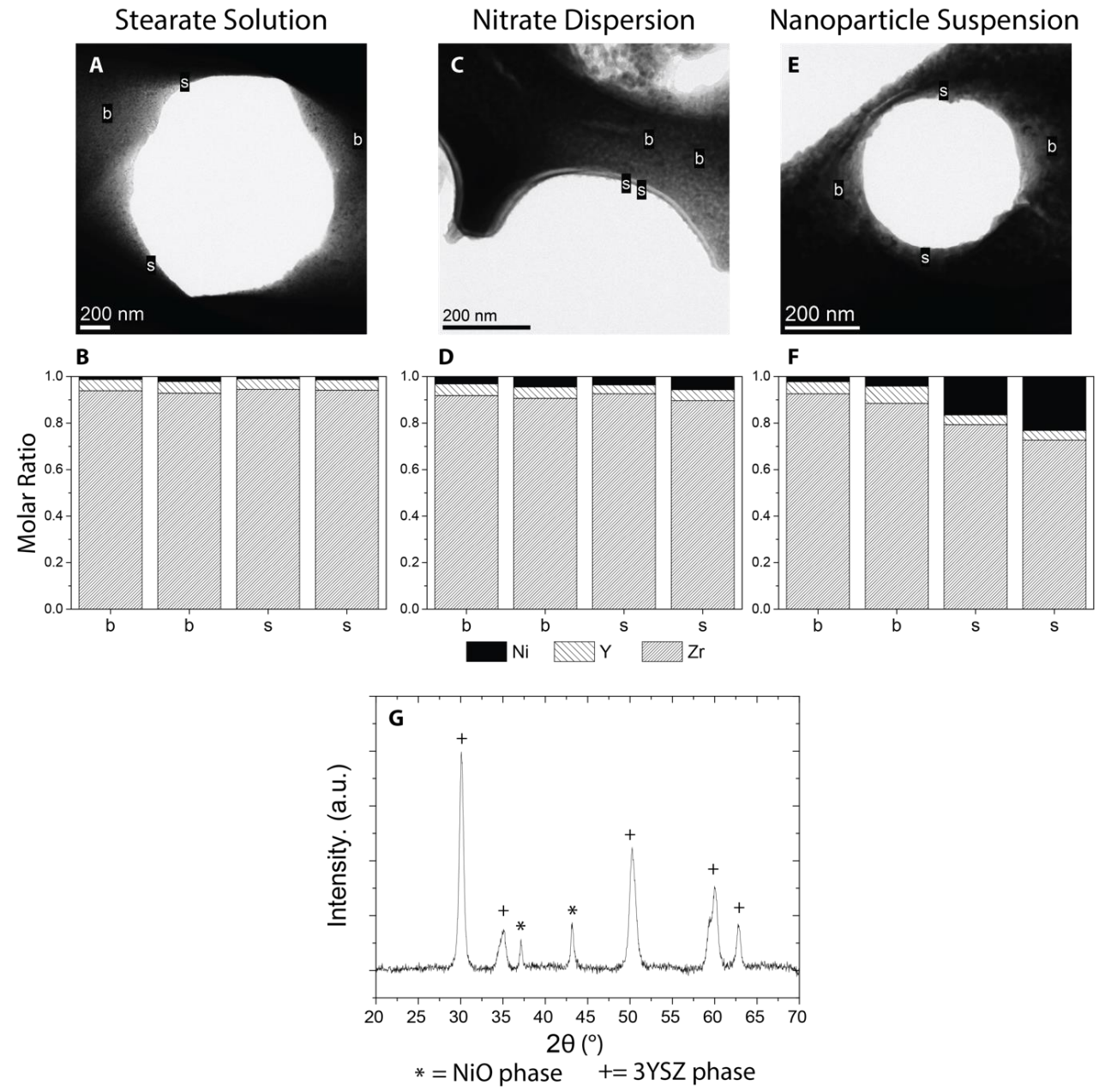

Figure 8. TEM and EDX point analyses of $(A, B)$ YSZ samples templated by fibers loaded with a Ni stearate solution ( $s=$ surface, $b=$ bulk), $(C, D)$ samples templated by fibers loaded with a Ni nitrate dispersion, (E,F) YSZ samples templated by fibers loaded with a NiO nanoparticle suspension. G) XRD pattern of the porous $3 Y S Z$ phase (marked with + ) loaded with NiO (*) from the nanoparticle suspension,, indicating a 2-phase mixture of 3 YSZ and NiO. 
The porous 3 YSZ sample templated with the NiO nanoparticle-containing fibers was selected for further tests. The phases of the 3YSZ-NiO mixture was confirmed by XRD as presented in Figure 8G. It must be noted that 3YSZ is tetragonal, that is why a peak split can be appreciated at 35 and $58^{\circ}$.

The active surface area was measured by hydrogen chemisorption. A commercial $\mathrm{Ni}$ YYSZ cermet, proprietary of ECN (the Netherlands), was used as reference for the sake of comparison. The as-prepared material and the commercial $\mathrm{Ni} / \mathrm{YSZ}$ cermet had similar active surface areas, i.e. $1.76 \cdot 10^{-3} \pm 1.1 \cdot 10^{-5}$ and $1.80 \cdot 10^{-3} \pm$ $4.0 \cdot 10^{-5} \mathrm{~cm}^{2} / \mathrm{g}$, respectively. However, the amount of nickel in the templated material (10.5 wt\%) was much lower than on commercial Ni/YSZ cermet (51 wt\%). By normalizing the active surface area over nickel content, the templated sample had an active surface area of $1.68 \cdot 10^{-2} \pm 1.0 \cdot 10^{-4} \mathrm{~cm}^{2} / \mathrm{g} \mathrm{Ni}$, which is 4.7 times higher than that of the commercial sample $\left(3.5 \cdot 10^{-3} \pm 8.0 \cdot 10^{-5} \mathrm{~cm}^{2} / \mathrm{g} \mathrm{Ni}\right)$.

The conductivity of the $\mathrm{Ni} / \mathrm{YSZ}$ sample from the $\mathrm{NiO}$ nanoparticle suspension was determined under SOFC anode working conditions, i.e. in a flow of $2.2 \mathrm{~mol} \% \mathrm{H}_{2} \mathrm{O}$ in $91: 9 \mathrm{~mol} / \mathrm{mol} \mathrm{N}_{2}: \mathrm{H}_{2}$. The impedances, presented in Figure 9 , have been corrected for the sample dimensions, but not for the porosity. In other words, the results relate to the overall sample including porosity instead of just the dense part of the sample. The impedance data could be modeled with a $\left(R_{1} C_{1}\right)\left(R_{2} Q_{2}\right)$ circuit (drawn lines in Fig. 9A). (RQ) represents a parallel combination of a resistance with a constant phase element $\left(\mathrm{CPE}\right.$, symbol ' $\mathrm{Q}$ ', with $\left.Y_{\mathrm{CPE}}=Y_{0} \cdot[j \omega]^{n}\right)$. When $n=1$ it represents a capacitance and when $n=0$ a resistance. A value of $n$ in the range of 0.8 - 1 is regarded as a pseudo-capacitance $[23,24]$. The $n$-value for $Q_{2}$ is $0.86-0.96$, indicating pseudo capacitive behavior. A clear assignment to specific transport processes (ionic conductivity, grain boundary conductivity, electrode dispersion) is as yet not possible. The scatter in $R_{1}$ and $R_{2}$ is rather large, 
however the sum of $R_{1}$ and $R_{2}$ shows little scatter in an Arrhenius graph. This sum is presented in Fig.9B as conductivity (Arrhenius expression of $\sigma \cdot T$ versus $T^{1}$ ), with:

$$
\sigma=\frac{L}{A\left(R_{1}+R_{2}\right)}
$$

$L$ is the sample length and $A$ the sample cross-section. In Fig. 9B also the ionic conductivity for TZ3Y is presented [25]. The corresponding activation energies (61 and $77 \mathrm{~kJ} \mathrm{~mol}^{-1}$ ) are reasonably close, but the conductivity of the templated sample is significantly lower. This high value clearly indicates that the conductivity is almost purely ionic in nature [26]. The significantly lower conductivity could be due to enhanced grain boundary resistance and/or electrode polarization. The low electronic conductivity will be a limitation for use as anode material. Further study is needed to elucidate the conduction mechanism and improve the properties of this material.
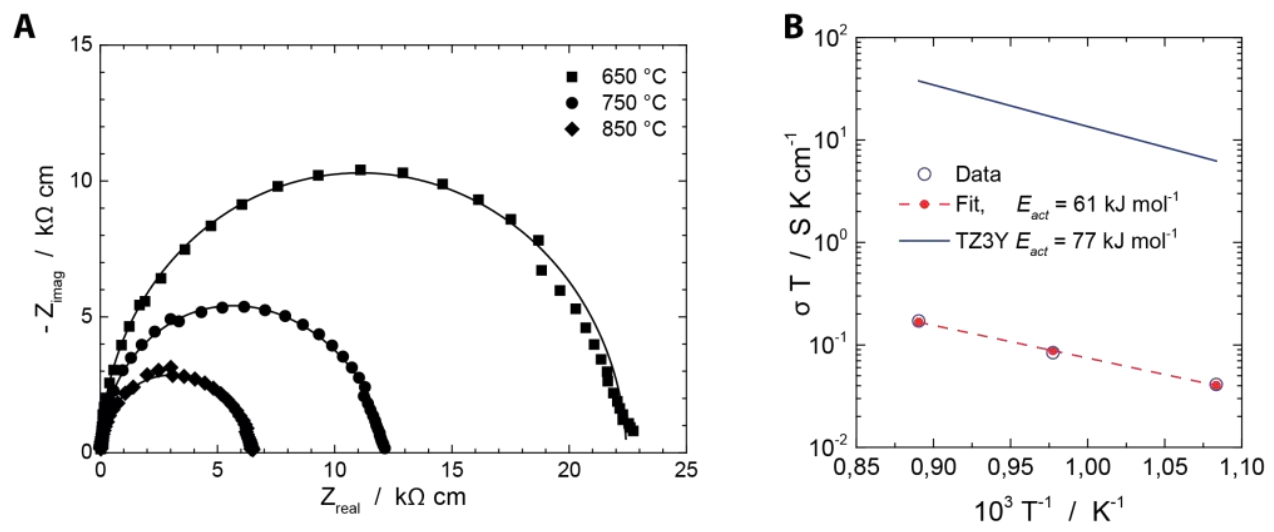

Figure 9. A) Three normalized impedances at different temperatures. B) Arrhenius-type graph of the conductivity $\sigma T$ versus $1 / T$, not corrected for porosity.

The increased active surface area was attributed to the high porosity combined with the small pore size and the controlled deposition of nickel [27]. Moreover, the controlled deposition of nickel on the surface ensures a high active surface 
area [27]. However, the electronic conductivity was found to be low. This may be attributed to one or two main causes. Firstly, the concentration of nickel present in the sample may be too low. Since Ni provides the transport path for electrons [27], a too low nickel concentration leads to isolated conductive patches instead of an electronically conductive connected network of particles on the pore wall. Secondly, anodes with excessive porosity have been shown to have decreased performance due to the lack of percolation paths for electrons and ionic oxygen [27]. It is worth noting that an increased porosity normally results in larger pores and smaller specific surface areas, unlike the method presented here. However, for SOFC anode application, a higher conductivity is required.

I believe that the electronic conductivity could be greatly improved, e.g. by introducing more species that are electronically conductive under reducing atmosphere. For instance, titanium ions have been reported to be electronically conductive when incorporated into zirconia $[28,29]$. Another option is to introduce nickel in the bulk. However, this strategy may not be the most desirable due to the aforementioned drawbacks related to nickel, being the catalytic deactivation and possible cracking due to volumetric expansion. 


\section{Conclusions}

Electrospinning was shown to be an excellent method to form sub-micron channel porosity in YSZ ceramics, and porosities up to $70 \%$ were demonstrated. The method offers high versatility to tune the final microstructure in terms of pore diameter, porosity, and pore directionality. Increased porosity can be achieved without enlargement of average pore size and loss of specific surface area. Moreover, the method allows local $\mathrm{Ni}$ catalyst deposition on the pore wall, leading to a much higher degree of dispersion of $\mathrm{NiO}$ compared to a commercial reference material. The concentration of deposited NiO remained relatively low.

I have demonstrated a simple and elegant method to form porous ceramics with controlled submicron porosity. The method opens the way to a wide range of possibilities to fabricate superior macroporous materials for several applications. Knowledge of electrospinning is extensive enough to allow a high degree of control over the arrangement of micro- and nanofibers, which is the critical factor in the present method. 


\section{References}

1. Boukamp, B.A., Fuel cells: The amazing perovskite anode. Nat Mater, 2003. 2(5): p. 294-296.

2. Studart, A.R., U.T. Gonzenbach, E. Tervoort, and L.J. Gauckler, Processing Routes to Macroporous Ceramics: A Review. J. Am. Ceram. Soc., 2006. 89(6): p. 1771-1789.

3. Chevalier, E., D. Chulia, C. Pouget, and M. Viana, Fabrication of porous substrates: A review of processes using pore forming agents in the biomaterial field. J. Pharm. Sci., 2008. 97(3): p. 1135-1154.

4. Hoa, M.L.K., M. Lu, and Y. Zhang, Preparation of porous materials with ordered hole structure. Adv. Colloid Interface Sci., 2006. 121(1-3): p. 9-23.

5. Okada, K., T. Isobe, K.-i. Katsumata, Y. Kameshima, A. Nakajima, and K.J.D. Mackenzie, Porous ceramics mimicking nature-preparation and properties of microstructures with unidirectionally oriented pores. Science and Technology of Advanced Materials, 2011. 12(6): p. 064701.

6. Sakka, Y., F. Tang, H. Fudouzi, and T. Uchikoshi, Fabrication of porous ceramics with controlled pore size by colloidal processing. Science and Technology of Advanced Materials, 2005. 6(8): p. 915-920.

7. Imhof, A. and D.J. Pine, Uniform Macroporous Ceramics and Plastics by Emulsion Templating. Chemical Engineering \& Technology, 1998. 21(8): p. 682-685.

8. Nie, L., J. Liu, Y. Zhang, and M. Liu, Effects of pore formers on microstructure and performance of cathode membranes for solid oxide fuel cells. J. Power Sources, 2011. 196(23): p. 9975-9979.

9. Divisek, J., R. Wilkenhöner, and Y. Volfkovich, Structure investigations of SOFC anode cermets Part I: Porosity investigations. J. Appl. Electrochem., 1999. 29(2): p. 153-163.

10. Tao, S. and J.T.S. Irvine, $A$ redox-stable efficient anode for solid-oxide fuel cells. Nat Mater, 2003. 2(5): p. 320-323.

11. Sun, C. and U. Stimming, Recent anode advances in solid oxide fuel cells. J. Power Sources, 2007. 171(2): p. 247-260.

12. Persano, L., A. Camposeo, C. Tekmen, and D. Pisignano, Industrial Upscaling of Electrospinning and Applications of Polymer Nanofibers: A 
Review. Macromolecular Materials and Engineering, 2013. 298(5): p. 504520.

13. Teo, W.E. and S. Ramakrishna, A review on electrospinning design and nanofibre assemblies. Nanotechnology, 2006. 17(14): p. R89.

14. Agarwal, S., A. Greiner, and J.H. Wendorff, Functional materials by electrospinning of polymers. Prog. Polym. Sci., 2013. 38(6): p. 963-991.

15. Veldhuis, S.A., A. George, M. Nijland, and J.E. ten Elshof, Concentration Dependence on the Shape and Size of Sol-Gel-Derived Yttria-Stabilized Zirconia Ceramic Features by Soft Lithographic Patterning. Langmuir, 2012. 28(42): p. 15111-15117.

16. Cui, W., X. Li, S. Zhou, and J. Weng, Investigation on process parameters of electrospinning system through orthogonal experimental design. J. Appl. Polym. Sci., 2007. 103(5): p. 3105-3112.

17. Fridrikh, S., J. Yu, M. Brenner, and G. Rutledge, Controlling the Fiber Diameter during Electrospinning. Phys. Rev. Lett., 2003. 90(14): p. 144502.

18. Jacobs, V., R.D. Anandjiwala, and M. Maaza, The influence of electrospinning parameters on the structural morphology and diameter of electrospun nanofibers. J. Appl. Polym. Sci., 2010. 115(5): p. 3130-3136.

19. Lee, J.H., H. Moon, H.W. Lee, J. Kim, J.D. Kim, and K.H. Yoon, Quantitative analysis of microstructure and its related electrical property of SOFC anode, Ni-YSZ cermet. Solid State lonics, 2002. 148(1-2): p. 15-26.

20. Delaforce, P.M., J.A. Yeomans, N.C. Filkin, G.J. Wright, and R.C. Thomson, Effect of NiO on the Phase Stability and Microstructure of Yttria-Stabilized Zirconia. J. Am. Ceram. Soc., 2007. 90(3): p. 918-924.

21. Stawski, T.M., W.J.C. Vijselaar, O.F. Göbel, S.A. Veldhuis, B.F. Smith, D.H.A. Blank, and J.E. ten Elshof, Influence of high temperature processing of solgel derived barium titanate thin films deposited on platinum and strontium ruthenate coated silicon wafers. Thin Solid Films, 2012. 520(13): p. 4394-4401.

22. Stawski, T.M., S.A. Veldhuis, R. Besselink, H.L. Castricum, G. Portale, D.H.A. Blank, and J.E. ten Elshof, Nanostructure Development in AlkoxideCarboxylate-Derived Precursor Films of Barium Titanate. The Journal of Physical Chemistry C, 2011. 116(1): p. 425-434.

23. Boukamp, B.A., A Nonlinear Least Squares Fit procedure for analysis of immittance data of electrochemical systems. Solid State Ionics, 1986. 20(1): p. 31-44. 
24. Boukamp, B.A., Electrochemical impedance spectroscopy in solid state ionics: recent advances. Solid State lonics, 2004. 169(1-4): p. 65-73.

25. Ferchaud, C., J.-C. Grenier, Y. Zhang-Steenwinkel, M.M.A. van Tuel, F.P.F. van Berkel, and J.-M. Bassat, High performance praseodymium nickelate oxide cathode for low temperature solid oxide fuel cell. J. Power Sources, 2011. 196(4): p. 1872-1879.

26. Chen, Y., X. Mao, H. Shan, J. Yang, H. Wang, S. Chen, F. Tian, J. Yu, and B. Ding, Free-standing zirconia nanofibrous membranes with robust flexibility for corrosive liquid filtration. RSC Advances, 2014. 4(6): p. 2756-2763.

27. Charge consequences in electrospun polyacrylonitrile (PAN) nanofibers. Polymer, 2005. 46(18): 7191.

28. Feighery, A.J., J.T.S. Irvine, D.P. Fagg, and A. Kaiser, Phase Relations at $1500^{\circ} \mathrm{C}$ in the Ternary System ZrO2-Y2O3-TiO2. J. Solid State Chem., 1999. 143(2): p. 273-276.

29. Kaiser, A., A.J. Feighery, D.P. Fagg, and J.T.S. Irvine, Electrical characterization of highly Titania doped YSZ. Ionics, 1998. 4(3-4): p. 215219. 
Chapter 7

\section{Conclusions and outlook}




\section{Conclusions}

The work presented in this thesis proves that electrospinning is a valuable tool to form functional ceramic materials for various applications. The most remarkable achievement was the formation of a flexible ceramic as presented in chapter 3. However, the methods developed to form arrays of devices on aligned ceramic fibers (chapter 5) or the controlled templating to form nanochannels in ceramic monoliths (chapter 6) demonstrate that the possibilities of electroctrospinning can be extended further than just making nanofibers mats.

As presented in chapter $\mathbf{2}$, the starting solution is the most critical aspect in electrospinning of ceramics. The starting solution defines the spinnability, but also has the largest influence on the fiber diameter. I have shown that the solution parameters can be tuned in order to fabricate specific compositions with controlled properties. The results obtained in chapter 2 provide enough information to be used as a quick guide to electrospin ceramic nanofibers.

In the chapters of this thesis, the fabrication of ceramic nanofibers with various compositions was reported, including yttrium doped zirconia (YSZ), calcium phosphates, nickel oxide, zinc oxide and metal precursor loaded polymer fibers. Each composition can be utilized in one of various fields of application. In particular, the potential of using electrospun ceramic nanofibers in the fields of biomedicine, electronics and energy conversion are illustrated in this thesis.

In chapter $\mathbf{3}$ and 4, I evaluated the potential of electrospun ceramic nanofibers for bone regeneration. Chapter 3 describes the improved microstructure and mechanical properties of YSZ which enhanced its osteogenic properties, while the bulk material is known to be bioinert. Therefore, it is likely that $\beta$ - tricalcium phosphate nanofibers, presented in chapter 4 , can be highly bioactive as they have an optimized composition and microstructure. In general, ceramic 
nanofibers seem to present an interesting alternative to form bone regeneration scaffolds. Other compositions, such as bioglass, have also been successfully tested $[1,2]$.

In the field of electronics, aligned nanofibers offer great potential for producing miniaturized devices with special functionality. Chapter 5 demonstrates the feasibility of producing arrays of aligned zinc oxide fibers in a short time by using the electrospinning technique. Electronic pads were fabricated onto the fibers to form transistors and a photoactive device. The possibility of producing electronic devices onto nanofibers with a flexible structure opens the possibility to form a new generation of flexible electronics and sensors [3-5]. However, the fabrication protocol and alignment of nanofibers has to be further optimized.

In chapter 6, a successful templating method for controlling the porosity and the catalyst deposition on the ceramic nanofibers was developed. Polymer fibers were used as sacrificial template to form nanochannel porosity in YSZ. The catalyst deposition was controlled to be on the wall of the pore when the fibers were loaded with catalyst nanoparticles. Although the material did not show the desired properties of a solid oxide fuel cell anode, which application was chosen as model to test the concept, the methodology can be adequate for other applications such as supported catalysts or filtration membranes [6-9].

Overall, electrospun ceramic nanofibers with tuned physicochemical properties and unique flexibility show a promising potential for the realization of superior devices in different fields of application. The work presented in this thesis provides more insight into the fabrication protocols of ceramic nanofibers, the characterization of the properties of these materials, and some of their potential applications. 


\section{Applications of ceramic nanofibers}

Ceramic nanofibers when used as a key component of a larger device, system or part of a technology, may provide a superior performance or functionality. Offering unique flexibility, such materials can be used in flexible electronics, sensing, composites, biomedicine, filtration or catalysis.

The field of flexible electronics is now dominated by polymers, since they can be shaped into a flexible device. However, polymers offer a inferior performance compared to the standard rigid devices. For instance, polymers offer lower electron mobilities, electrical conductivity or piezoelectricity than the ceramic equivalents. One of the main constraints of the current generation of flexible electronics is the limited thermal stability of polymers. Polymers cannot resist the temperatures utilized for depositing highly electroactive materials, so other alternatives are currently in use. Being adequately flexible, ceramic nanofibers mats may provide a suitable alternative to polymer-based flexible electronics. Since these materials can resist elevated temperatures, they allow the deposition of electroactive materials and formation of highly efficient flexible electronics.

Due to their large surface area and the possibility to be coupled to electrodes as illustrated in Chapter 5, ceramic nanofibers are an excellent candidate to be used in sensing applications. The large specific surface area provides more active sites for sensing, while the signal can be transmitted through the electrodes. The composition of nanofibers can be modified to form a range of different sensors.

Composites consisting of polymers with ceramic fillers are gaining interest in the market, especially due to their improved thermal and electrical performance [10]. However, the ceramic loading reduces ductility and flexibility, resulting in the formation of a brittle material. A homogeneous distribution of ceramic fillers is also hard to achieve, since the particles tend to agglomerate [11]. The flexible 
ceramic non-woven mats are a promising alternative to form composites with high content and homogeneous distribution of the ceramic fillers.

As shown in chapters $\mathbf{3}$ and $\mathbf{4}$, nanofibers show a great potential to be used in biomedicine. However, further biological testing must be done to confirm this assumption. This work is outside the scope of material science as it involves animal and clinical testing. It must be also noted that YSZ might not be the most suitable composition for implants. $\mathrm{Y}^{3+}$ has been reported to leach out of the crystal structure leading to brittleness, when YSZ was used in vivo [12].

Finally, filtration and catalysis are promising fields for ceramic nanofiber applications. Non-woven mats of electrospun polymeric fibers have been proven to be excellent membranes [13]. They allow a high flux, due to the high porosity, while having a relatively small pore size of few microns. Flexible ceramic nanofibers allow forming similar filters that can resist high temperature during filtration, as well as provide catalytic activity when loaded with catalyst particles.

\section{Scaling of the production}

Scaling the production of the nanofibers is another further challenge for ceramic nanofibers. Indeed, there are some industrial electrospinning setups, for instance Nanospider ${ }^{\circledast}$ from El Marco, in which a needleless electrospinning approach is used [14]. However, electrospinning might not be the best candidate for producing nanofibers on industrial scale. Although electrospinning is a worthy alternative for lab scale, the main drawback lies in the use of a static electrical field, as presented in chapter 2 . The electromagnetic field requires the use of expensive and toxic solvents in order to stabilize the free ions.

Force spinning emerges as an alternative technique to form nanofibers mats without using high electrical voltages. The principles of force spinning and 
electrospinning are similar. However, forcespinning drags a viscous solution into nanofibers using centrifugal force instead of electromagnetic force [15]. This technique allows higher nanofibers production volume than electrospinning. FibeRio commercializes FiberEngine ${ }^{\circledR}$ tools with an output much larger than any electrospinning tool [16].

The main advantage of electrospinning compared to force spinning is the possibility of forming highly aligned arrays of nanofibers. Therefore, further engineering must be done on the force spinning setup in order to get large-scale outputs of aligned nanofibers.

Another challenge for the upscaling of ceramic nanofibers are health related issues. Nanomaterials are classified as potentially dangerous. Especially when shaped in the form of non-biodegradable nanofibers. High aspect ratio materials that cannot be rejected by the body itself can cause lung cancer, similar to asbestos. The potential hazards of ceramic nanofibers should be investigated in order to fully elaborate protocols for their safe handling.

As a concluding remark, ceramic nanofibers may be found in commercial products in the near future. Studies show that the market for nanofibers is rapidly evolving $[17,18]$. Ceramic nanofibers, with their superior characteristics, are expected to grow rapidly into industrial production in the forthcoming years. 


\section{References}

1. Lu, H., T. Zhang, X.P. Wang, and Q.F. Fang, Electrospun submicron bioactive glass fibers for bone tissue scaffold. Journal of Materials Science: Materials in Medicine, 2009. 20(3): p. 793-798.

2. Wei, X., Z. Daming, and C. Jiang, Fabrication and in vitro biomineralization of bioactive glass (BG) nanofibres. Nanotechnology, 2007. 18(13): 135601.

3. Ding, B., M. Wang, X. Wang, J. Yu, and G. Sun, Electrospun nanomaterials for ultrasensitive sensors. Mater. Today, 2010. 13(11): p. 16-27.

4. Wu, H., D. Lin, R. Zhang, and W. Pan, ZnO Nanofiber Field-Effect Transistor Assembled by Electrospinning. J. Am. Ceram. Soc., 2008. 91(2): p. 656-659.

5. Chen, X., S. Xu, N. Yao, and Y. Shi, $1.6 \mathrm{~V}$ Nanogenerator for Mechanical Energy Harvesting Using PZT Nanofibers. Nano Lett., 2010. 10(6): p. 21332137.

6. Hoa, M.L.K., M. Lu, and Y. Zhang, Preparation of porous materials with ordered hole structure. Adv. Colloid Interface Sci., 2006. 121(1-3): p. 9-23.

7. Studart, A.R., U.T. Gonzenbach, E. Tervoort, and L.J. Gauckler, Processing Routes to Macroporous Ceramics: A Review. J. Am. Ceram. Soc., 2006. 89(6): p. 1771-1789.

8. Boukamp, B.A., Fuel cells: The amazing perovskite anode. Nat Mater, 2003. 2(5): p. 294-296.

9. Chevalier, E., D. Chulia, C. Pouget, and M. Viana, Fabrication of porous substrates: A review of processes using pore forming agents in the biomaterial field. J. Pharm. Sci., 2008. 97(3): p. 1135-1154.

10. Wong, C.P. and R.S. Bollampally, Thermal conductivity, elastic modulus, and coefficient of thermal expansion of polymer composites filled with ceramic particles for electronic packaging. J. Appl. Polym. Sci., 1999. 74(14): p. 3396-3403.

11. Wu, C.L., M.Q. Zhang, M.Z. Rong, and K. Friedrich, Tensile performance improvement of low nanoparticles filled-polypropylene composites. Compos. Sci. Technol., 2002. 62(10-11): p. 1327-1340.

12. Depprich, R., C. Naujoks, M. Ommerborn, F. Schwarz, N.R. Kübler, and J. Handschel, Current Findings Regarding Zirconia Implants. Clinical Implant Dentistry and Related Research, 2014. 16(1): p. 124-137. 
13. Sundarrajan, S., K.L. Tan, S.H. Lim, and S. Ramakrishna, Electrospun Nanofibers for Air Filtration Applications. Procedia Engineering, 2014. 75: p. 159-163.

14. Marco, E. Nanospider. 2016; Available from: http://www.elmarco.com/.

15. Sarkar, K., C. Gomez, S. Zambrano, M. Ramirez, E. de Hoyos, H. Vasquez, and K. Lozano, Electrospinning to Forcespinning ${ }^{T M}$. Mater. Today, 2010. 13(11): p. 12-14.

16. FibeRio. FiberEngine. 2016; Available from: http://fiberiotech.com/.

17. Nanofibers: Technologies and Developing Markets. BCC Research, 2010.

18. Nanofibers Technologies Market - Global Industry Analysis, Size, Share, Growth and Forecast 2012 - 2018. Transparency Market Research, 2012. 


\section{Summary}

Ceramic micro and nanomaterials have gained interest by the research community during last decade. They have contributed to improved performance in the fields of catalysis, biomedicine, energy and electronics. Amongst these ceramic materials, nanofibers have shown good potential; their large aspect ratio enhances several material properties.

This PhD thesis focuses on the development of novel functional ceramic fibers and nanochannels. Different materials were fabricated, characterized and tested in different applications.

The ceramic nanofibers and nanochannels have been fabricated by combining two techniques: electrospinning and sol-gel processing. The goal of this thesis is to investigate the influence of the fabrication process of different ceramic nanofibers on their properties, and subsequently to explore their potential in different fields of application.

In order to be able to tune the materials' microstructure and properties, the process of preparing ceramic nanofibers by electrospinning was studied. The role of the solution properties on the process was thoroughly investigated as it controls most of the process and determines the feasibility of producing fibers. It was demonstrated that the fiber diameter, morphology and alignment can be modified. The possibility of preparing nanotubes is also shown. Such understanding was essential to design materials in order to enhance their performance in different fields of application. 
A flexible ceramic consisting of a 3\% Yttrium Stabilized Zirconia non-woven mat of fibers was designed to be used as scaffold for bone regeneration. Although that ceramic material is inherently rigid and bioinhert, it was proven that it can be turned into flexible and offered an improved bioresponse towards osteogenesis when shaped into nanofibers. Similarly, the possibility of shaping $\beta$ tri calcium phosphate, a well-known bioactive material for bone regeneration into nanofibers, has been demonstrated. The final grain size in the resulting nanofibers was smaller than reported elsewhere, which is thought to be caused by the fibrous morphology.

Ceramic nanofibers also showed potential to be applied in the field of electronics. The fabrication of aligned $\mathrm{ZnO}$ nanofibers, and the semiconducting and photoelectric properties were studied in field effect transistor and UV detector applications. It was proven that electrospinning can be used for rapid fabrication of large area electronic devices.

A novel method to fabricate porous ceramics with controlled catalyst deposition is also presented. Polymer nanofibers were used to template ceramic materials and form nanochannels. 3\% Yttrium Stabilized Zirconia / Ni cermet was chosen a model material to be used in the field of solid oxide fuel cells. Nevertheless, the same method can be extended to other fields.

In conclusion, it can be stated that ceramic nanofibers offer a promising alternative in different fields of application. However, the scaling and commercialization still remains a challenge. 


\section{Samenvatting}

Keramische micro- en nanomaterialen staan onder toenemende belangstelling van de wetenschappelijke wereld gedurende de laatste 10 jaar. Ze hebben bijgedragen aan verbeterde prestaties van materialen op gebieden zoals katalyse, biomedische toepassingen, energie en elektronica. Uit deze groep materialen, zijn de nanovezels in het bijzonder opvallend qua potentieel: de hoge aspect ratio verbetert diverse materiaaleigenschappen.

Dit proefschrift richt zich op de ontwikkeling van vernieuwende keramische nanovezels en nanotubes. Diverse materialen zijn gemaakt, gekarakteriseerd en getest in ene verscheidenheid aan toepassingen.

This PhD thesis focuses on the development of novel functional ceramic fibers and nanochannels. Different materials were fabricated, characterized and tested in different applications.

De keramische nanovezels en nanotubes zijn gemaakt gebruik maken van twee technieken: electrospinnen en sol-gel processing. Het doel van dit proefschrift is gericht op het onderzoeken van de invloed van het maakproces op de eigenschappen van de verschillende keramische nanovezels. Vervolgens is ook het potentieel van deze materialen in de diverse toepassingsgebieden onderzocht.

Om het beïnvloeden van de microstructuur en de eigenschappen van de materialen mogelijk te maken, is het electrospinnen van de keramische nanovezels dieper onderzocht. De invloed van de eigenschappen van de startoplossingen is diepgaand bestudeerd omdat deze het sterkst van invloed zijn op het proces en de mogelijkheid om de vezels hoe dan ook te maken. De 
diameter, morfologie en uitlijning van de vezels blijkt hierdoor te kunnen worden beïnvloed. Ook is aangetoond dat nanotubes kunnen worden gemaakt. Dit begrip over de invloed van de eigenschappen van de oplossing is essentieel om materialen zo te ontwerpen dat hun eigenschappen voor de verschillenden toepassingen kunnen worden verbeterd.

Een flexibel yttrium (3\%) gestabiliseerd zirconia keramiek in de vorm van nietgeweefde vezelmatten is gemaakt om als substraat voor botregeneratie te functioneren. Ondanks dat het keramische materiaal inherent rigide en bio-inert is, hebben we kunnen aantonen dat het kan worden omgevormd met behulp van nanovezels tot een flexibel substraat waarop verbeterde bot aangroei kon plaatsvinden. Op een vergelijkbare manier hebben we ook $\beta$-tri-calcium-fosfaat, een bekend bioactief materiaal voor botregeneratie in nanovezels, kunnen vormen. De uiteindelijke korrelgrootte in de resulterende nanovezels was kleiner dan tot nu toe gepubliceerd. Dit wordt toegewezen aan vezelachtige morfologie van het substraat.

Keramische nanovezels hebben ook potentieel te worden toegepast in elektronica. Het maken van uitgelijnde $\mathrm{ZnO}$ nanovezels en hun halfgeleidings- en foto-elektrische eigenschappen zijn onderzocht in de toepassingen voor fieldeffect transistors en UV detectoren. We hebben aangetoond dat electrospinnen kan worden ingezet voor snelle productie van onderdelen met grote oppervlakten.

Tot slot wordt een vernieuwende methode beschreven om poreuze keramiek te maken waar gecontroleerd katalysator materiaal in kan worden afgezet. Polymere nanovezels zijn ingezet om als substraat te dienen om keramische nanotubes te vormen. Yttra (3\%) gestabiliseerd zirconia / Ni cermet is onderzocht als 
uitgangsmateriaal vanwege de toepassingen in vaste stof brandstofcellen. Dezelfde methode kan echter ook voor andere toepassingsvelden worden ingezet.

Keramische nanovezels bieden een veelbelovend alternatief voor een groot scala aan toepassingen, maar het opschalen van productie en vercommercialiseren blijft voorlopig de grootste uitdaging. 


\section{Acknowledgements}

This section is going to be by far the most read part of the thesis and the one that makes me happiest, as it means that it is over!

Although this is my PhD thesis, I would have not been able to go this far without the help and support of many people. First of all, I would like to thank the members of my graduation committee, Guus Rijnders, Rob Lammertink, Louis Winnubst, Karen De Clerk and Lorenzo Moroni, for reviewing this thesis. Obviously, without a committee there is no graduation. Secondly, I would also like to extend my special thanks to my supervisors Andre and Bernard. Andre gave me the opportunity of exploring and fed my creativity, while providing guidance both in scientific and personal level. Bernard, sorry for not working with Impedance Spectroscopy in the end. Still, I must say that I really learnt a lot from you, every time I had a theoretical question you were there to give a detailed explanation. I would also like to express my special gratitude to all the current and former technical staff of IMS: Henk, Dominic, Marion, Jose, Karin, Laura and Kees. We all know that your assistance is essential to perform research. In many cases, I have considered you my heroes! Furthermore, I should also thank the heroes of the NanoLab, Mark, Rico and Gerard, I had a lot of fun working with you guys! I would like to acknowledge my students: Bahruz, Vera, Luis, Michel and Kristianne. I had lots of fun working with you all and I learnt a lot from you too. I also want to thank my colleagues for all the brainstorming and good times we had. I have to say that I learnt a lot from all the chemical brothers (and partners): Sjoerd, Coralie, Anotny, Pablo, Joanna, Wouter M., Maarten, Roy, Alessandro, Suresh, Huiyu, Rogier, Petra, Tomek, Nicolas, Jeffrey, Wouter V. I also appreciate all the physicist (the 
dark side) in the group and the work they do. Many thanks to Rik, Dave, Nirupam, Kurt, Jaap, Tasos, Ruud, Peter, Tom, Thomas, David, Duus, Evert, Gert Jan, Mark, Tjeerd, Lin, Zhaoliang, Minh, Ben, Daniel, Anirban, Ron, Alim, Werner, Brian, Bouwe, Jose, Jasper, Mathijn. Although I did not get to work so much with you, it was always a pleasure to have you around! Finally, I want to acknowledge all the help from my main collaborators, especially the ones at TR group, Paul, Honglin, Giuseppe, Lorenzo and Pamela. I would not have been able to finish my PhD without you!

My PhD resulted in Eurekite, where I also have lots of funs and I am learning day by day from my colleagues and network. Bahruz, Pavel, Zaskia, Umit, Roger, Marcel, Max, Chaim, Marijn, Ellen, Ties, Milli, Ray, Dave, Alain, Peter, Frank, Bas and Gianina, many thanks for making my days so interesting!

I have been in Enschede for 6+ years and, obviously, I did not spend all the time doing my PhD. During these years, I got to meet many interesting people that in one way or another influenced the way I am nowadays and consequently the way I did my PhD.

I first came to Enschede to do my master thesis at the Membrane Technology Group. It was there when I decided to stay here for my PhD. I have very good memories from that time and also people associated to those. Can, Aran, Laura, Olga, Michelle, Dimitris, Pavlina, Maria, Gregory, Xavi, Damon, Saghar, Enver, Frank, Ana, Jigar, Annisa, Elif and Geraldine, many thanks!

Also, during my first years in Enschede, I used to hang out at the Farmhouse. Those good old times when I was not pretending to be that serious... Alejandro, Darren, Sovan, Javi, Quique, Aykan, Antonia and Alexi, we should have a reunion some time! Some other people that I also met during my Erasmus exchange were Tony, Celia, Merve, Eneda and Carlos, hope you all the best! 
During my years around here I tried to be also active in playing music (even though it is not happening anymore). I first started with Mediterranean United together with Cagri, my favourite Bob Marley. Later on, I joined Rimon. We recorded few songs and had several gigs but overall, I learnt a lot from my bandmates Rimon, Joey, Jochem, Diego and Marc. When Rimon was over, I joined the Kapsalons together with Juancho, Mireia, Joep and Filipe. It was cool to go back to the punk roots with you! And talking about my punk roots, I must mention CK and my first band-mates Adri, Marc, Kabreta, Oli, Xinot and Chaki. They had a big influence in shaping me to become the person who I am nowadays.

While living in the Netherlands, I also got the passion for playing football. It first started as a fun activity and later on, it became Soup-A-Stars. Damir, Alex, Emilio, Tom, Teo, Adi, Erwin, Afonso, Jorge, Lasse, Teo and others, many thanks for dominating! Soup-A-Stars also merged with the futsal team of Drienerloo 4, where I also got to know some other great guys: Mark, Muharram, Sertan, Ozlem, Joris.

Besides doing the PhD and so many activities, I also had time to go out with friends. I want to highlight the group of the Latins (Rodrigo, Ignacio, Afroditiy, Adriana, Dimitris, Eva, Jair, Laura, Dani, Edu) and the Italians (Matteo, Aga, Caterina, Federico, Virginia, Giorgio, Marco, Kasia, Alex, Samuele, Riccardo, Giuseppe, Alessia, Miriam, Marcello, Bea, Franceso). I had lots of fun with you all! And talking about fun... I cannot ignore the good time we had at our house in Pegasustraat with all the people around: Matteo, Bobo, Manana, Lorenzino, Can Arslan, Zuzana, Nico, Marina, Ramazan and Rob!

Nowadays I also spend lots of time in Maastricht with former TR people. Angad, Ivan, Febriyani, Lissy, Lisette, Parthi, Elahe, Corina, Nick, Anne, Maaike, Jorg, David, Joana, Andrea, Joao, Rita, Paul, Mijke, Erik, Jingwei, Carlos, Daniel, 
Afonso, Ziryan, Nardin, Rong and Honglin thanks a lot for welcoming me in the group!

During my PhD I also met the girl of my life: Niloofar! I am so happy to have been brave and go for it back in time. I remember those days that I made up any excuse to spend time around TR. You have made me so much better and I hope you feel the same way about me. I also want to thank Niloo's family (Narges, Nastaran, Maman and Ambili) for treating me so well.

Via Niloo, I also got to hang out with Mojtaba, Sadaf, Mitra, Sia, Mohammad, Ana, Nastaran and Ehsan. Many thanks for being so welcoming and teaching me about Persian culture.

Finally, I would like to thank my dear friends from back home. Arnau, Guilera, Pau, Jaume, Maria, Isabel, Roger, Eva, Amela, Laurins, Anna, Quim, Joana, Puig i Suko: som un sol front, el Sala al cor! Vosaltres sou aquells amics de sempre amb qui puc ser el Celio al natural (i ho sofriu). Sou la meva família no de sang. També vull expressar la meva gratitud al Gili, la Eli i el Rodrigo de la UB. Hauríem de veure'ns mes sovint!

I parlant de família, no em puc oblidar de la meva família de sang: Papa, Mama i Esther. Moltes gràcies per haver-me donat suport i per no queixar-vos massa perquè no truco gaire sovint. Us estimo! 


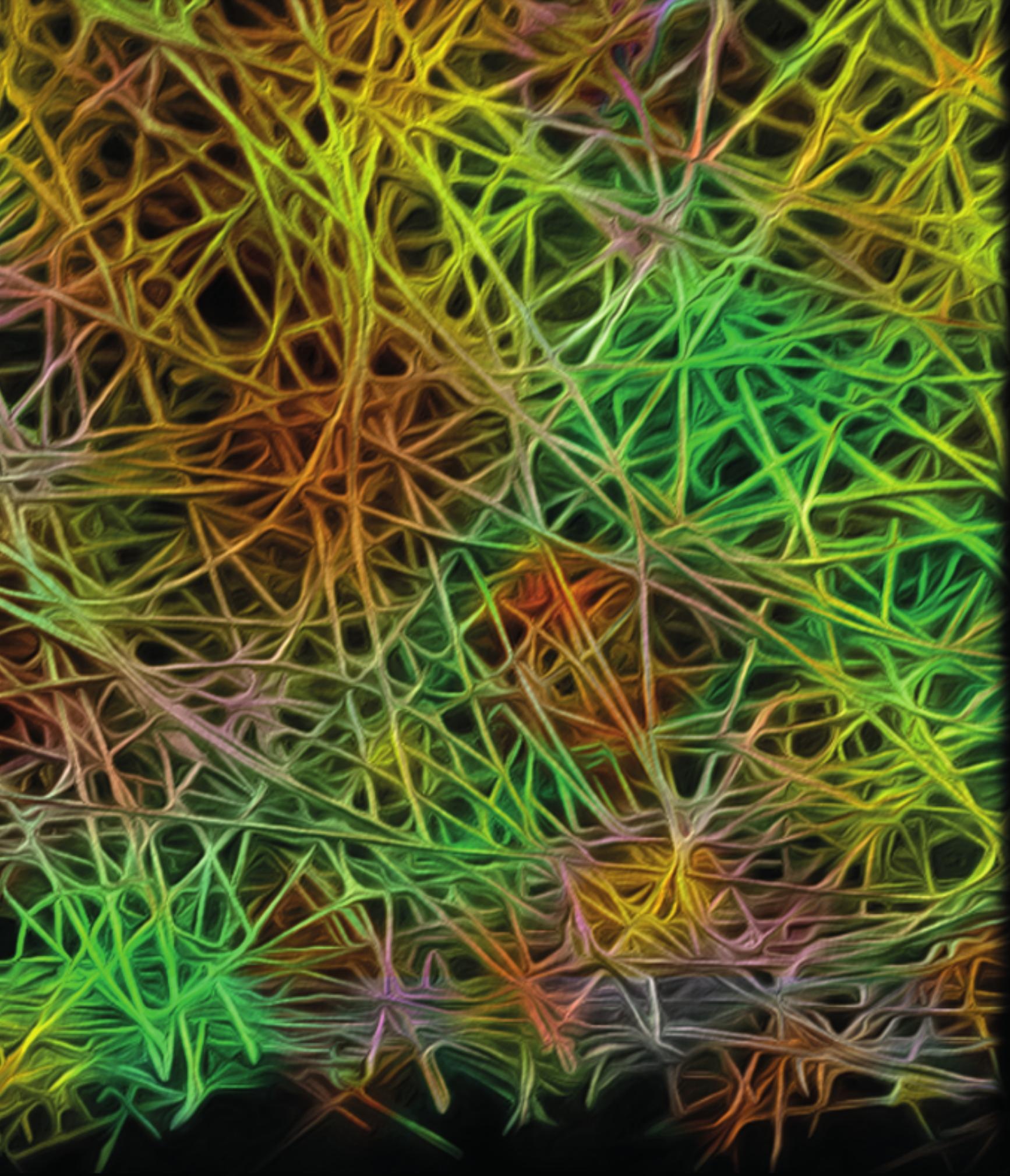

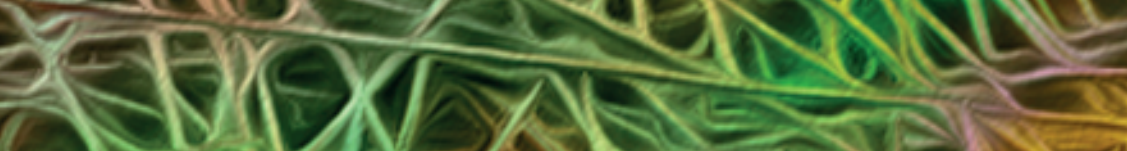

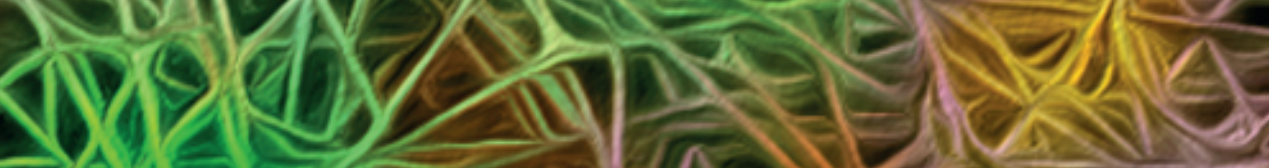

\section{ISBN:978-90-365-4254-8}

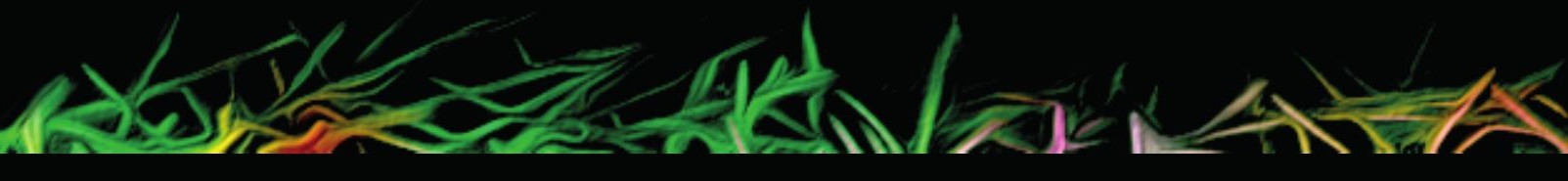

